$x=$ 
(1) 191908

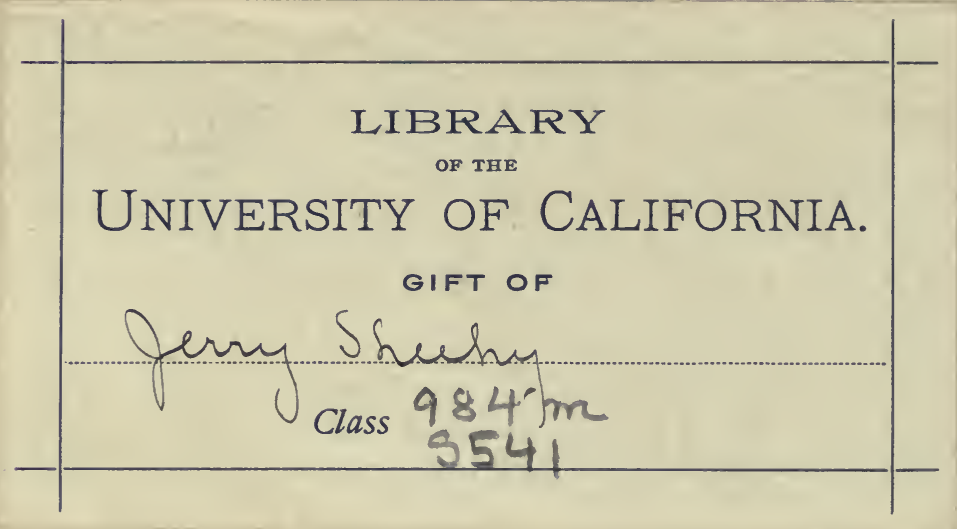


3H. h. 6.68owees, Newvis:

Buts, flew-13-

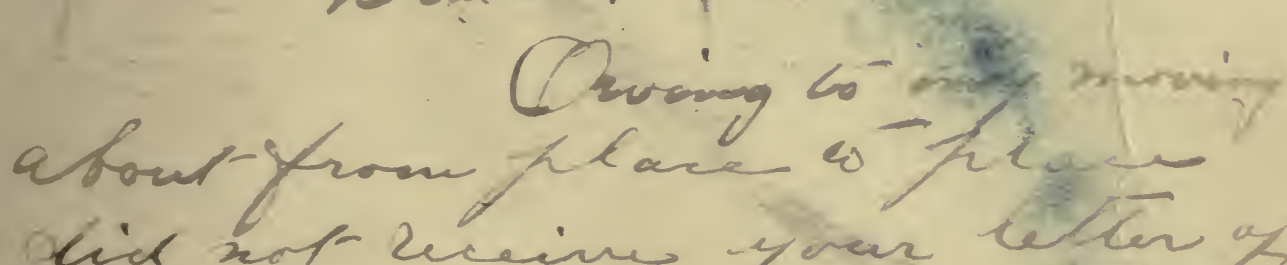
aid nof- havin yoven water of bept $=24$, entil a day on two ay Il owever I have mailed a

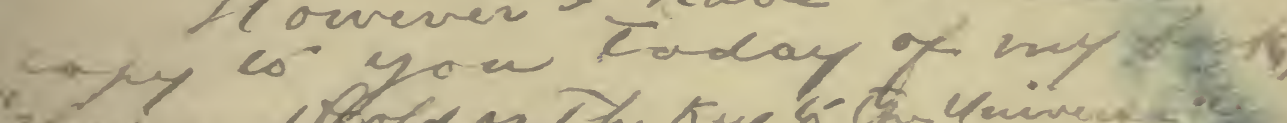

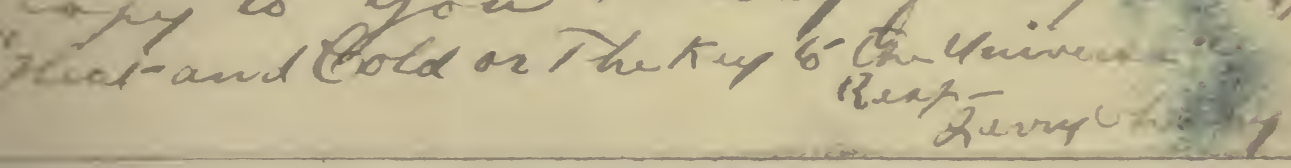


Digitized by the Internet Archive in 2007 with funding from Microsoft Corporation 


\section{HEAT AND COLD}

\section{OR}

\section{THE KEY TO THE UNIVERSE}

By

JERRY SHEEHY

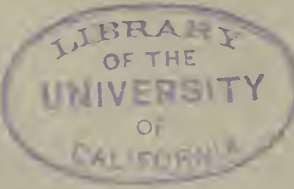

PUELISHED BY THE AUTHOR 
Entered according to Act of Congress, in the year 1908, by Jerry Sheehy, in the Office of the Librarian of Congress, at Washington.

DEMPSTER BROS.

PRINTERS

447 MINNA STREET

San Francisco, California 


\section{INTRODUCTORY.}

The object of this work being to show how erroneously the scientific world has dealt with the problems bearing most pointedly upon the lives and liberties of men. The mode of thought without foundation often multiplying a wrong in trying to acquire right. The scientific world always looking for precedent as the foundation of judgment; all the while acting upon a precedent that was founded on the most cruel and unjust foundation. The scientific world has attempted to serve two masters; and have proven how hard it is to get results from two masters. The scientist of to-day attempts to inquire into a question, but while inquiring he also tries to make it conform with revelation Bibular; in consequence, he has made a fool of himself. He is not a scientist or a true disciple of the Bible. He is nothing multiplied by nothing. That is the best we can make out of the average scientist when weighed in the scale of true science or true revelation Bibular.

The author was lead to question the judgment of all men, owing to a great injustice being inflicted by the judgment of fellow men; in questioning the judgment of men, he found all men throughout all ages from the dawn of history until the present time to be the same; namely, corrupt, unjust and dangerous to each and all. The judgment of men throughout all historic periods 


\section{INTRODUCTORY.}

tends to prove that the best in man is always the most likely to be the cause of his being persecuted by his fellow man. Ignorance always being jealous of knowledge. In consequence, ignorance being more prevalent, crushes knowledge whenever it raises its head above the debris. All the while, man has worked his own destruction by crushing the best of his kind. $\mathrm{He}$ seems forgetful in his injustice to the fellow men of the fact that injustice to one individual, when weighed in the scale of law of precedent, carries injustice to his own door. He does not know that each injustice brings doom nearer to himself and his brother.

The work deals with things earthly first, and weighs all matter and movement in the scale of passing action, as observed in the simplest daily observations. In other words, the work builds the foundation on what we see in nature instead of groping into the shadows, for precedent, built on floating shadows. The author begs that the reader will question every line of the work, and weigh it in the scale of his own observations, and add to same every truly scientific fact discovered by man by same means of study of passing action.

The work picks man up in the gases in space, and traces his development by true law down to the dawn of history, giving cause, reason and mode of change throughout. Same with many animals in a cursory way; in fact, the general process applicable to all animal life. The work also deals with man to infinity when weighed as to futurity; traces his course beyond the range of the average intellect into the future. Yet 


\section{INTRODUCTORY.}

all capable of proof conclusive in the scale of passing action as observed by the simplest of our children.

The work leaves no query behind or before; all leading questions of value to mankind are answered in a cursory way. The why? whence? and whither? are all answered. The work could have been continued indefinitely, but the author believes that sufficient has been revealed to make the law at the bottom of all clear to any intelligent reader. He has endeavored to make it as plain as possible by leaving out scientific modes of expression wherever possible. 



\section{HEAT AND COLD}

CHAPTER I

\section{THE BEGINNING OF THINGS EARTHLY.}

Out of a mist of gases the creation of worlds was but the work of nature along the lines of our daily observation in the powers of nature displayed upon the earth. Because all of nature's products can be reduced to the gaseous form by the application of a sufficient amount of heat. And as all the stars in the heavens conclusively show that the heat is present in the heavens. And all the planets show by their appearance that they each had a greater temperature at a former time.

Consequently, we must infer from what we see on our own planet that they each had a greater temperature in years gone by. Greater temperature spells gases at some distant period in the world's history.

Now, to transport ourselves to the gaseous world and let us live in fancy within the changes that had to transpire within the conditions then existing. First, gases made from the reduction of one property of a condensing temperature would condense into a solid or semi-solid property, in advance of the gases of a property of a higher temperature of condensation. And in consequence, this is now our law for the making up of our planet out of the gases.

Second, our researches into the qualities displayed by nature render it very evident that matter is inde- 


\section{HEAT AND COLD.}

structible. And further, the same researches render it quite positive that matter cannot be made out of nothing.

Using these facts as a foundation to lay our work upon, then the question arises. Did the properties now in existence which have different temperatures of precipitation exist at the time of the gaseous period? To this question we must answer, yes, they existed in the light of all knowledge: We being able to return matter to that state. Having to accept the existence of all the properties now in existence as a matter of fact during the gaseous period. Now, the temperature of precipitation of the various properties in relativity becomes our study. And the miraculous transformation coming to pass during the cooling process within the particles of matter, must surrender within that code.

To meet this problem of change in the particles, we have two forces at work which, by modification in time and place, render all the work quite clear to us. Cold being a cohesive force and heat being a dispersing power, what more natural than the modification of the two forces bringing about every change which nature has to be questioned?

Gravity, animal life, compression, and, in fact, any and every phenomenon in nature will surrender their history in the making to the relative powers exhibited by these two forces, when weighed in the light of their every-day powers as manifested beneath the gaze of the common being, man.

During the period that our planet existed in the 


\section{THE BEGINNING OF THINGS.}

gaseous form, it was necessary that it did fluctuate for millions of years in space. Always portions of same impinging against the colder portions of space. The portions that impinged against the colder portions of space cooling in conformity with the respective temperatures of precipitation in relativity. The cold tending to condense the particles that would condense at the highest temperature first. And each property following in conformity with its temperature of condensation in relativity. Cold always acting upon the property that would tend to cohesion, or congeal at the greatest temperature.

These properties being on the outer boundaries of the body of gases owing to the cold being more severe in the outer portions. Consequently they began to form around the body of gases within the center. The central portion of the gaseous body being a burning mass, and in consequence having to be fed. That is to say, the flame having to have pabulum to keep up the fire: Consequently the precipitating particles of matter out on the cold borders condensing on the exterior cut away the food from the flame. The flame having to have an outlet to exist, and in consequence it became smothered by the enwrapping particles. The particles on the exterior, which were being congealed by the action of the cold, were not only forced to settle to a zone in relativity conforming to the given temperature of precipitation, but were acted on by cold obliquely, tending to work off at a tangent. This power of the cold acting obliquely on the particles forced the matter to begin 


\section{HEAT AND COLD.}

revolving on the exterior; and the revolving power, when once in motion, was accelerated by the cold during each revolution. Thereby we find the earliest movements in the particles that formed the planet brought about by the cold.

The condensing process continuing until it cut off the chance of escape of the burning particles within the center of the globular mass. The central portion being on fire, necessitated the chance of escape of the burned particles of gases in the form of sunlight or light. The cutting off of same tended to smother the gaseous nucleus, by the burning out of the gases before the final smothering of the flame. Thereby we have traced our planet down to the shell of condensing matter around a gaseous nucleus of great size, and minute attenuity.

Having imprisoned the gases in the center of the property in nature admitting of solidity at the highest temperature. Now our study will be the precipitation of the properties that could not possibly be precipitated at the given temperature of the first crust that imprisoned the gases. All other properties having a temperature of precipitation beyond the first crust could not be precipitated at the time of the forming crust, because the heat that would admit of precipitation of that one property would drive the properties of a more volatile character far off in the heavens. And they each would have to await in suspension until the body cooled sufficiently to admit of precipitation, within their respective temperatures of condensation.

The gases being imprisoned within the first crust 


\section{THE BEGINNING OF THINGS.}

being burned to a very minute form, owing to having been burning at the time of the imprisonment by the forming crust, caused the body as a whole to become more buoyant after the closing. Being more buoyant as a body, the planet was influenced from without by two forces. The sun began to act on the whole as a body, owing to having a solid exterior, and drove it farther away. Being driven farther away, it became acted on by the cold with great vigor. The cold reducing the crust during each revolution in temperature, and causing the precipitation of other properties held in suspension in conformity with their respective temperatures of condensing. The crust, as it began to accumulate in weight in portions through the precipitation of material coming within the range of temperature, became reduced in portions more than in others. And in consequence there were properties of different temperatures being precipitated in the same location, owing to the cooling action of the crust coming in contact with the particles in suspension.

The crust being so cold during the revolving against the colder portions of space during the semirevolution in opposition to the action of the sun, it caused the compounding of properties by the revolution within zones where they were held in suspension.

During each revolution the body cooled sufficiently to admit of the precipitation of particles, and continued throughout all the different properties of matter in conformity with their respective temperatures of precipitation. 


\section{HEAT AND COLD.}

During each succeeding revolution of the body it cooled, so as to admit of the precipitation of other properties, until finally the period when properties already within the crust acted as catalyzers in causing the precipitation of other properties yet in suspension, thereby making the various compounds we find in nature, including that of organic life. But it took millions of years for the planet to cool sufficiently to admit of the precipitation of the properties that went to making up of the organic life.

As the body cooled off, it continued to be compressed in the particles that constituted it, and in consequence the power of attraction of the body was increased. During the earlier period in the formation of the crust, the only attraction being the cold on the particles of matter in conformity with their relative temperatures of precipitation.

It was further aided by the movements in revolving through generating a power toward the center, and the final and last force was in the form of stored cold in the form of compression within the particles that make up the planet.

The planet finds a medium of existing in space through the interaction of the two forces of heat and cold, each aiding in suspending the planet. But the two forces are aided in the placing of the planet by the gaseous center or nucleus within the body of the planet. The heat of the sun drives the planet as a whole away until the congealing action of the cold becomes equal to the driving power of the sun on the given body. The 


\section{THE BEGINNING OF THINGS.}

planet being formed around a gaseous nucleus and being not at all unlike a balloon. In consequence, the powers of the sun in driving the same away are limited to the weight of the body as a whole taking into consideration the gaseous center. The sun can drive it only until the cold becomes a congealing power equal to the sun as a lifting power. In consequence, the planet chooses a place of movements in a line of demarkation between the forces of heat and cold, varied by the size of the gaseous nucleus. Consequently, we find little trouble in accounting for the position of the planets without inverting the order of nature as observed within our daily surroundings.

A study of the means of burning matter here on our planet will render conclusive proof that a fire cannot live without an outlet. The fire needs a means of carrying off the minute reduced particles. Particles reduced by being burned to the minutest form must make room for other particles to come in to feed the flame. The minute properties burned or dispersed to the minutest form exert a pressure outward upon the particles of matter, and will deprive the admittance of properties whose weight will not overcome the pressure exerted outward. Thereby, we find that the fired gases in the central part of the body of burning gases were conclusively imprisoned when reduced to the minutest form by heat. Gases could not possibly be displaced in space by solid particles only by pouring the solid out of a great pot. If they were poured out of a great pot, still they would fall through space until sufficient heat was 


\section{HEAT AND COLD.}

generated to reduce the properties to gases again. So every law of nature proves that the gases could not cool into a solid. That the earth is not solid. No man dare say that he can show a means whereby a law admitting of proof within our reach, can bring about the solidifying of gases into a solid in space.

But the law is very clear whereby we may prove the imprisonment of gases within the relative laws of condensation of the different properties within space. And not only imprison the gases but prove why it is inevitable that they should be imprisoned in time as long as matter can be had in sufficient quantities. And in doing so, accept our positive experiments on the, laws of matter as the base of our action, or as our fundamental law in bringing about the imprisonment of same. Not going off into the mists for a means of imprisoning the gases, but instead using as our base the laws of matter as proved to exist here within the reach of the humblest of mankind. Laws that are unimpeachable and irrevocable as far as man is concerned.

The center of the burning gases being surrounded by the first property forming into a solid at the greatest temperature in worldly elements. Then the smothering of the fire in the gaseous center owing to having no outlet for the reduced articles. What will be the result in the premises, considering that the particles of matter imprisoned are very attenuated properties of matter owing to the reduction by the great heat? It appears in the premises that the gases will cool by moving toward the crust by the loss of heat occasioned by 


\section{THE BEGINNING OF THINGS.}

the cutting off of the escaping reduced particles. And a further reduction will be occasioned by the cooling of the crust, causing the gaseous center to settle toward the crust, through the laws of matter congealing in conformity with different temperatures.

The continued exposure of the outer crust of the planet to the extreme cold, owing to the gaseous center being large in proportion to the amount of solid matter forming the crust, and in consequence the sun driving the whole body to the outer border of the gases bordering extreme cold, would in that case occasion the cooling of the crust greatly during each semi-revolution of the body. Cooling of the crust greatly would occasion the congealing of the gases from the center or nucleus of gases toward the cold portion of the crust during each revolution. The continued congealing of the gases toward the crust as the body hastened to cool would cause the nearest thing we have in nature to a genuine void or vacuum within the center of the globe or planet. The crust having reduced the greater part of the gases to a solid consistency before the crust thickened to any great extent, and in consequence the heat generated in after years when the crust began to thicken through the accumulation of great bodies of matter on the outside by precipitation from the gaseous form in conformity with their respective temperatures of condensation, would not produce heat sufficient to reduce the gases which had solidified within the center to the gaseous form again. In consequence the body would 


\section{HEAT AND COLD.}

be far more buoyant than in the case of a very attenuated gas forming the center.

A study of the different temperatures of the freezing point in the various properties of matter, coupled with the temperatures of boiling point, and a further consideration of the point in each property where it is wont to evaporate or distill, or both, will readily give the clue to nature's law in the building of planets. Not only the building of planets but the building of organic matter of all orders including that of man.

It will be found that the properties congealing at the greatest temperature in worldly elements form the lower strata in worldly formation as discovered by man in his studies of geology. And so on up through the different strata to the surface or the organic properties. Each strata conforming to the different temperatures in conformity to their respective congealing relations.

Of course, in the study of surface, it must be taken into consideration that the crust of the earth has been broken by an outside jolt in the greater portion of the surface, and the shaking up must be taken into consideration in the judgment of strata. (On another page in this work we will deal with the jolt.) But in places on the earth's surface, where the surface was not broken by the inflicted jolt, it will conform to the order of the properties in relativity with their respective temperatures of congealing or solidifying. The highest temperature in solidifying forming the lower strata, and so on up to the age of organic properties being precipitated. 


\section{THE BEGINNING OF THINGS.}

We will also find in the lower strata, built up of organic nature, a close affinity in the properties of matter next preceding it on the earth's crust. Thereby giving or shedding light on the means of the first organic properties coming about upon the planet.

It will be found by a study of the natural order of the properties of the earth's crust, that is to say, where unaffected by the jolt from the outside force (or by water), the properties next preceding the organic properties were of a nature that would act upon the particles of matter as catalyzers, causing properties yet in suspension to come to earth through their chemical affinity. The properties precipitated next preceding causing properties in suspension to unite and form into organic properties. Thereby forming compounds, and hence life. A further study will reveal the presence of properties next preceding organic properties in the order of reduction in relativity in temperature. The dividing line that marks the junction of the two properties in the earth's strata, where undisturbed, will conform in proving that organic properties were inevitable in the order of nature. Not chance, but the inevitable law of nature. One of nature's immutable laws manifest in the true order of nature, and adaptable to the condition and the times.

As to the formation of life, it was just as impossible to evade the generation of life in the circumstances as to stop the precipitation of the properties within the temperature.

Taking into consideration the strata, or studying 
same as a means of getting at the truth of nature's law, it must not be forgotten that variations in the beds or strata were brought about by the compounding of properties admitting of compounding at great temperatures owing to the revolution of the planet. The planet having revolved at great speed owing to the semi-solid consistency of its crust of minute thickness and great buoyancy. Being of a semi-solid consistency not unlike a liquid, it was greatly affected by exposure to the cold or to the heat during each revolution, and in consequence their powers were greater than now manifest on solid properties. Each revolution cooling portions of the surface more than others, caused the precipitation of properties conforming to the temperature of the crust in the location, and for that reason a mixing of the strata into minute thickness in portions, while in others forming compounds within the temperature of crust acting upon the properties yet in suspension, causing same to be precipitated and joining in forming compounds. Thereby accounting for different portions of the earth's crust at the same period in worldly history forming into different forms of strata, or a different earthly formation at the same depth. in the earth's strata. The revolution of the earth effecting the cooling of the crust caused different formation to be coming into existence at the one and same time in worldly history.

So the study of strata necessitates the consideration of locality with regard to the source of heat generated from the outside body driving the planet away. Again 


\section{THE BEGINNING OF THINGS.}

it necessitates the consideration of portions of planet exposed to the greatest extremes in cold during the revolution away from the sun or driving power.

In other words, topographical conditions as exposed to us to-day when undisturbed are our best monuments of past actions in the cooling process; that is to say, when not broken by the outside jolt.

High mountains tend to prove that the underlying strata was cooled at an earlier age in worldly history, causing the piling up of immense mountains of matter owing to having the cooling period earlier in each particular property of matter. Of course to be considered when undisturbed by the jolt from outside forces. The primary cause of mountains being the cooling of crust in the particular portions of surface. The cooling of crust necessitating the precipitation of matter being held in the volatile form in conformity with respective temperatures of precipitation. The further precipitation causing a cooling to hasten in the premises owing to the thickness of same or distance from heat below crust, and the deposit of each succeeding layer of the properties of matter upon the crust at the given point in the cooling process occasioned another precipitation of property of a lower precipitating temperature in worldly properties. And so on down the order of the properties of matter in relativity until the age of organic properties, or the age of life as we know it in the form of animal and vegetable.

The organic properties that came into existence during the precipitation on the forming mountains being 


\section{HEAT AND COLD.}

of course changed by the action of water owing to water being prior in precipitation to our study of the properties of life. The organic properties being washed down into the valleys by the waters falling upon the mountains, thereby changing the forming strata after the age of the precipitation of the compound forming water. 


\section{CHAPTER II}

\section{GRAVITY.}

There is no theory set up by man in support of a scientific fact so thoroughly vulnerable as the hypothesis of attraction of gravitation. It appears to be so simple in the proof of its absurdity as to render all of man's versions along all lines of thought questionable, owing to having even accepted it for truth for a day, not to say centuries.

When the greatest minds for upwards of three centuries were led to accept such rot, what can we expect of the judgment of the average man on questions of depth, questions touching their own welfare and that of fellow-men's?

We should, when questioning matters of such importance as the powers displayed by our sun in the heavens, bring back our reasonings to the simplest surroundings here on earth, and by observing the powers here displayed by that luminous body, just merely extend them. We should consider the powers displayed by the sun on the properties of matter here before our eyes, and add to that observation the powers displayed by any form of heat within the range of our knowledge.

We should remember that the sun displays a tendency here before our eyes to disperse matter into its elements. Then we should remember that in order that 


\section{HEAT AND COLD.}

the sun should attract in the distance, it should exercise the same powers where we have access to its power on the properties of matter. If it attracts big things, how does it get to the point of change from the way it works on small particles of matter? Where is the dividing line between its dispersing power and its attracting power? Does it attract when it forces water off in the clouds? Does it attract when it lengthens the steel rail? Does it attract when it develops the gas into a greater bulk? Does it attract when it expands the flesh on your finger so as to make your ring too tight on a hot day? Does it attract when it forces down on the seed buried in the earth and causes it to sprout or expand into an organic body? Where, oh, where does it attract?

Then we should revert to the powers exercised by cold within the range of our observation, or immediate surroundings. And by doing so find what properties it tends to display. Cold under our immediate observation tends to the union of all matter. The steel rail that no amount of heat could be induced to contract will visibly contract beneath our immediate gaze. The finger ring worn on any member of our hands will be found to be too large when we have been exposed to the cold. The smoke or reduced particles of gaseous substances raising above a fire where they have been dispersed by heat, will visibly make for the earth when exposed to cold. These properties are not forced back to earth by the sun, but instead by the cold upon the particles of their structure. They being lighter than air, must be condensed by the cold in order to fall to earth. 


\section{GRAVITY.}

Why should we go off into space looking for a zone where the sun changes its power of dispersal and becomes a cohesive force, when we have a force here on earth that can do all the attracting? A force that we positively know attracts, by thousands of years of observation on the action of its powers on the properties of matter? And infinitely extended these two powers we observe on earth in the action of the sun in expanding, and cold congealing, will account for every phenomenon in nature.

We need not grope off into the shadows for a zone where they swap horses and then continue their journeys in opposite directions to eternity, either. Infinitely extended, the two powers will manifest no change.

The theory of attraction of gravitation requires the subversion of the powers exercised by the two forces within our immediate surroundings, and in consequence falls to the ground. We cannot accept one power for cold beneath our gaze and another off in the distance. We cannot accept one power for heat under our own observation and allow it to be traded off for another horse to ride home on. We will not allow any one to force us to believe that heat, while dispersing here beneath our vision, changes off in the distance and pulls like a tug-of-war team.

The power we observe in heat coupled with the powers we observe in cold will account for every phenomenon we encounter in nature, and we do not need to subvert the powers of either one of them. Heretofore it has been the accepted hypothesis that the sun 


\section{HEAT AND COLD.}

through its attraction held every one of the planets in place. In order that the sun should attract those planets it must of necessity swap horses in the middle of the stream, and for that very reason the sun attracts no planet in the heavens. But instead, drives them away. Acting in the distance just as we observe it acting in our immediate surroundings.

The only reason that that style of reasoning stood all these years must be attributed to the gullibility of man in allowing himself to be imposed on by the names of men considered great having accepted the reasonings. If man will only wake up to the fact that men are deemed great often when, in fact, very commonplace, owing to coming before the public during their lives, the average great man being nothing more than a man that had become noted during his life by getting into public confidence through circumstances unavoidable as the precipitation of matter in a given temperature.

These great men, having read the reasonings of the original propounder of the theory of gravity, accepted it without question, their minds being too narrow to even question the matter. You may have asked either one of them what was gravity. And he would perhaps have answered thus: "A power generated by the sun upon matter tending to union, or in fact a power within matter tending to attract all matter."

If you had asked him what made gravity, he would have died before he could have divined the problem, 


\section{GRAVITY.}

and yet lived three score and ten years all the time studying the problem.

The portion of his answer dealing with matter attracting matter would be correct during certain conditions, but the conditions being changed, the particles of matter would work the reverse; therefore that part of the question and answer would have no weight in the sense he handed it out.

Gravity in its infancy was nothing but cold, but later its powers were advanced by the compression of the particles acted upon by the original gravity. In that case the compression acted as power in storage, or cold in storage tending to help the original force in attracting. Again the attraction was advanced through the revolution of the unit or body forming a center of gravity. This third property displayed in attraction has borrowed power from heat in adding to the power within the unit. This borrowed power is the portion of the work of revolution borne by the heat center upon the particles of matter in or on the unit. Then a fourth power becomes added in making up the final worldly attraction as we know it. This fourth power becomes manifest in the cooling of the gases within the center of the body of the unit or planet. They having cooled, tends to make a vacuum within the center of the planet, and in consequence the particles of matter bear toward the center with great force, and this last makes the final limit in the summing up of what gravity has been derived from. Of course, throughout all this in the cooling process, the chemical affinity exercised within the 


\section{HEAT AND COLD.}

properties of matter became a power within certain temperatures. But their powers were limited to conditions generated by a modification of the forces of heat and cold.

And as to the answer that the average great man would make as to matter attracting all matter, he must acknowledge that cold and heat must make the condition whereby matter can attract all matter. Again he must acknowledge that at no time can the condition be so as to admit of matter attracting all matter. The only way it could do so would be at the point of absolute zero in the measure of cold, and then it would not be matter attracting matter, but instead cold attracting all matter.

So, again, matter attracting must fall to the ground without cold and heat modify each other in different regions of the earth, so as to allow one a sphere of influence and the other a like sphere of influence, to bring about the necessary temperatures in space. Thereby heat and cold must be acknowledged as the powers that matter must look to for condition where it can exercise its attraction.

The worldly attraction or gravity as applied to a planet or body in the heavens does not exist beyond the centripetal force generated by the planet revolving on its axis. The planet beyond that line of demarkation tends to throw away by its centrifugal force generated by the revolving body.

The centrifugal force generated by the revolution of a body tends to preserve it from foreign properties 


\section{GRAVITY.}

being thrown upon the surface, and is one of the greatest of nature's provisions for the protection of life and continuation of the planet as a whole. The centrifugal force is always greater than the congealing action of the cold, and in consequence no property can come in by the action of gravity within that line of demarkation until the power has been reduced by the action of cold upon the body in itself. Cold or gravity properly must act on the body in itself by reducing the revolution on the axis in order to force the line of demarkation generated by the revolving mass. In cooling the body it reduces the power of revolution. In reducing the power of revolution it renders the power to throw away less. And in consequence of time will overcome the power to protect itself by the contrifugal force generated in revolving.

The properties entering from outside space within the line of demarkation generated by the revolution of the planet are always forced in by the action of heat reducing particles to such minute particles as to defy the power of the body generated by revolution in throwing away. Heat reducing particles so minutely attenuated in form by separation of the particles that they pass the force tending to throw away, like sunlight passing through water. The particles being so minute as not to be palpable to the forces throwing away.

These particles coming in by the separating properties of heat are acted upon by the cold within the centripetal range of the planet, and are congealed within their respective temperatures. 


\section{HEAT AND COLD.}

Zones of gravity are made by the establishment of a nullification of the forces of heat and cold, or, a zone of demarkation between their respective forces. One being equal to the other in the premises, and in consequence the particles of matter coming to that line become condensed within that line until sufficient weight has been accumulated to cause the body to sink toward the body buoying it away or the force of heat. These zones being the cause of bolides whenever the body has not been formed around a gaseous center. In the case of a gaseous nucleus the body will continue to adapt itself to the medium between the two forces as it sinks toward the sun. But where the body has been formed into a solid within the zone of demarkation between the forces of heat and cold, it will sink toward the sun as soon as it has accumulated weight enough to defy the power that propels it. Having sunk toward the heat source or power that propels it, it will continue to move with greater force until reduced to minute particles or to weight that will be equal to the zone of propulsion.

Heat and cold acting in concert bring about the phenomenon of revolution of the planet, and in that way are the makers of the power generated to the center from the revolving of the body. Heat detaches particles from one side and cold precipitates particles on the side diametrically opposite, and in consequence destroy the equilibrium of the body, and cause it to revolve on its axis. A continuation of this process means continual revolution. Particles of an elastic quality 


\section{GRAVITY.}

like gases, air and water and like properties will be acted on more readily, and in consequence the speed will be in conformity with the quantity of like properties. When the body was all gaseous it was much more affected by the action of cold when exposed to the same than it is to-day when most all solid. The same can be said of the action of heat at that period, and in consequence, great speed in revolving at that period of world building.

The action of the compass needle when observed in proximity of a great wall or mountain can be attributed to the action of a form of gravity born of compression. And the amount of varation will conform to the amount of substance compressed or the amount of compression within the given body so affecting.

A few degrees lower temperature in the body so effecting will be found to vary the needle, thereby proving the compression to be the pure and only attraction in the given case. Or will lend one more proof of the fact that cold is gravity and can be represented by storage in carrying on its good work of attraction.

Where compression has been generated by the action of heat it will lend additional proof of the fact of compression being a form of gravity, but the storage generated by heat will not attract all properties like the compression brought about by cold. The compression brought about by heat is a compression of one of the most minute forms of matter. The particles being reduced to minute form and compressed within a limited space attracts particles of chemical affinity. 


\section{HEAT AND COLD.}

The compression brought about by cold affects every property within nature regardless of what it may be made of. The only variation being in the case of the compound water, in which cáse the cold contracts until reduced to $3^{8}$ degrees of Fahrenheits Thermometer, and in that case, water expanding until frozen. But while it expands, yet it attracts all other properties and also water to the expanding mass.

Compression, magnetism and gravity are all properties of the same nature when considered from an earthly standpoint.

To better understand this problem we should study the conditions existing about the poles of our planet. Cold being the true gravity and being extremely applied with regard to our planet at the poles; in consequence we have compresion more thoroughly manifest in those regions. Compression being stored cold, or cold in storage; hence we have cold extremely applied to our planet, plus cold in storage extremely applied. Result appears in the sum of magnetism extremely applied to our planet.

What more natural than the sum of magnetism would be our answer when we find that compression is the maker of every attraction from gases to the present time?

Magnetism, born of cold through its tendency to the union of matter. Cold acting on the properties of matter through its tendency to cohesion or advanced condensation.

It is the simplest of earthly observations to note 


\section{GRAVITY.}

that cold tends to contract all matter; only exception in the face of four degrees in the action of water, and in that case it yet attracts all other matter while it expands in itself. Infinitely extended cold will be found to exercise the same properties of contraction.

One of the simplest observations in nature is the tendency of heat to disperse all matter, and infinitely extended it will manifest the same qualities.

The heat of the sun by day upon a body of water causes a separation of its particles, causing the same when reduced to minute particles to assume the aerial flight owing to least resistance. The same phenomenon occurs when we throw a heavy stone into a tank of water; the earth being too heavy to be displaced by the jolt inflicted, it then becomes necessary for the water to act along the lines of least resistance. Consequently up to the air.

The most convincing proof of cold being the true and only gravity in fact, may be had by a test of any gaseous substance. It will be found that the gas will defy what we call worldly attraction, or what the savants call universal gravity. But subject the gas to a rigid test of cold, and you will be rewarded by its obeying the real law. Add sufficient cold and you will be rewarded not only by its being obeyed but in addition you may unite the gas into a solid.

The scientific world has been hoodwinked for hundreds of years by trying to prove all great questions by mathematical computation. The scientists have gone on figuring from a base and carrying a sum of 


\section{HEAT AND COLD.}

figures to eternity, all the time oblivious of the fact that figures weigh nothing only the base. The base being wrong, the figures tend to multiply the wrong. The savants never thought of how valuable subtraction would be in the case. They never thought of how valuable it would have been to take one from one and derive nothing. In other words take the void base from the bottom of the figures. 


\section{CHAPTER III .}

THE REVOLUTION OF THE EARTH ON ITS AXIS, WEIGHED IN THE LIGHT OF PRESENT CONDITIONS OF SURFACE.

The revolution of the earth when weighed in the light of present conditions of surface proves conclusively that the phenomen is brought about by the interaction of heat and cold. The earth being surrounded by a large volume of air and a large body of water, each being easily influenced by the action of either of the powers, heat or cold. In order that we may well judge of the effect of the power of the sun and the action of cold upon these bodies of air and water, we will make our point of observation in mid Pacific between the United States and the Chinese Empire. The Pacific being the greatest body of water and in consequence it will be the best place to take our observations from.

Let our hour of contemplation be at the hour of the rising of the sun above the expanse of the waters of the Pacific. As the sun apparently leaps out of the water it spreads its buoyant rays over a vast body of air and water, which has been cooled by the action of the cold during the night previous. That cooling action upon the particles of matter that make up water 


\section{HEAT AND COLD.}

and air is working toward the rising sun, and in consequence has to be governed by the heating rays of the sun. As the hours roll on the sun pours forth its heating rays upon the body of air and water until the rays of heat overcome extremes upon the particles of matter in the given zone of observation. This zone in the rising of the sun being the governing force in the regulation of the revolving process, keeping the revolution regular, offsetting extremes in speed.

When the sun arrives at the meridian at noon it has completely neutralized (extremes in) the action of the cold upon the particles of matter occasioned by the cohesive processes of the night previous. That is considered from our point of observation. And from the time the sun arrives at the meridian until night it becomes manifest with great vigor bearing toward the east.

As the sun dips toward the west in the setting it bears toward the east with great force upon air and water considered from our point of observation, and has no opposing force to deter it in moving easterly on the particles of matter. In proof of our contention of no power opposing the sun in its power easterly we will consider the conditions in mid Atlantic at the given time. The sun having set in the Atlantic long before its lowering on the western portion of the $\mathrm{Pa}$ cific. In consequence the last portion of the Atlantic toward the east has been deprived of the heat the longest and in consequence cooled first causing the water to cool toward the eastern portion of the body. 


\section{REVOLUTION OF THE EARTH.}

Cooling toward the east in the Atlantic spells in this case a tendency to union of matter in the particles of the water in the Atlantic at the time the sun lowers to the west in the Pacific; and in consequence must be considered as working in harmony with the power of heat at the given time exercised in the Pacific.

At the time the sun propels with greatest force eastward on the particles of water in the Pacific, and cold works eastward on the particles of water in the Atlantic, the sun is exercising the rising governing power on the land bodies of Asia. Because the sun in the rising has to neutralize the (extremes in) cold at all times in some portions of the earth's circumference.

Thereby we find that heat and cold act in union all around the earth in carrying on the revolution of the planet; and always in harmony toward the east governed by the rising of the sun at some particular portion of the earth.

As the sun dips to the west below the Pacific in the setting it bears with vigor on the part last acted upon, and a reduction in temperature begins on the eastern portion of the Pacific owing to the sun having long since passed from bearing on that portion of the body of water. And in consequence the tendency of union of matter in the particles of water in the Pacific will be easterly owing to the action of cold. Or in other words the cold takes up the burden which the sun had dropped and in so doing continues the good work eastward. 


\section{HEAT AND COLD.}

In the summing up of the matter we find that heat and cold act in harmony eighteen hours out of every twenty-four, and the remaining six hours we find the sun working upon the particles of matter as a governor in overcoming extremes in revolution of the body.

The contraction upon land and also its expansion when subjected to the action of the sun's rays tend to aid in carrying on the revolution of the body, but has no such power as the expansion of the waters and air. of the planet.

Nature has a provision for every eventuality and has more than one safeguard in carrying on her work. In the case of cold upon the particles of water, cold tends to contract until the waters lower below 38 degrees of Fahrenheit's thermometer and then it tends to expand. Expansion being in this case an additional safeguard or governor in the revolving process to aid the sun in keeping the revolution regular. Thereby tending to guarantee the equilibrium of the body as well by stopping a rush to a particular zone or portion of the earth. No extreme of climate can possibly bring about disaster until the final extinction of one of the forces that carry on the work.

During the absence of the suns rays of propulsion on either ocean it will be found that the greater portion of the body remains within the range of the cohesive tendency of the waters of the globe. Thereby proving that the waters are a necessity in the aiding of the revolution of the body as a whole. Should the revolution become abnormal and more than the gov- 


\section{REVOLUTION OF THE EARTH.}

erning power of the sun could offset the additional power of the solidifying of the waters would tend to reduce the speed. The waters being exposed to the cold's action more in the case of greater speed and in consequence would drop below the range of cohesiveness. Having dropped below that range the water would cut off two ways from the revolving power. First by lessening the amount of matter to be acted on in favor of revolution and while lessening same work diametrically opposite from the power displayed at the higher temperature.

The sun acts upon the aerial properties just as it does upon the bodies of water; it buoys toward the east upon the body of air after it tends toward the west in the setting. The air, like the waters of the globe, becomes acted upon owing to being plastic, but the air being much more plastic, and in consequence is subject to many minute forces which would not affect the waters. Any small disturbance in the equilibrium of forces on any portion of the earth may affect the currents of the air. The currents of air being a flowing in of the plastic properties to regulate the disturbance, or bring about equilibrium of forces.

At sunrise it may be observed the greater portion of the time the currents of air go before the sun in the rising, owing to the propelling rays of the sun becoming effective upon the more plastic properties before it really affects bodies of matter of a more solid consistency. But in all cases the air does not go before the sun owing to some derangement of the forces in 


\section{HEAT AND COLD.}

some other portion of the earth's surface that becomes manifest with greater vigor upon the particles than the rising of the sun. Air being the more plastic property connected with our planet and in consequence it runs in to regulate forces wherever there has been any disturbance. Air also tends to show how nature has always provisioned for any necessity to carry on its good work, having so many means of guaranteeing the regulation of the proceedings.

In no place will nature be found wanting in more than one way of remedying a disturbance in the order of its laws. It has not one safeguard for any emergency but instead many applicable to the same disturbance. Nature has a great many means of reserve force ready to apply in case of an emergency, and applicable to any emergency that may come to pass. Concluding the matter we find in the face of all these conditions that the action of heat on the particles of matter aided by cold upon the particles of matter, viewed in the face of present conditions cause the revolution of the earth on its axis. 


\section{CHAPTER IV}

\section{THE VOYAGE OF THE EARTH AROUND THE SUN AND ITS-EFFECT ON THE CLIMATE.}

The location of the earth with regard to the sun has a very material effect upon the climatical conditions of our planet. The earth being forced to revolve on its axis by the action of heat and cold, it likewise is forced to move around the sun from the same cause. In each case the greatest force being manifest upon the plastic properties of matter, that is water and air.

Air and water being the more plastic properties of the earth's makeup and in consequence they become our study.

When the earth passes to the north of the sun marking the equator out against space north of the sun, leaving the sun south with regard to the northern hemisphere; then the rays of the sun become diagonally applied with regard to the northern hemisphere.

The sun in this case being situated south of the equator with regard to the planet and its rays become powerfully effective within the southern hemisphere bearing down upon the waters in that portion of the earth. The southern hemisphere being for the greater part water and in consequence the sun's rays force the water off in the form of clouds. The sun being diagon- 


\section{HEAT AND COLD.}

ally effective with regard to the northern hemisphere owing to the location of the equator with regard to the sun. And in consequence the water forced into the cloud form and coming within the range of the rays of the sun bearing diagonally toward the north, and is in consequence driven off toward the north. When carried away north by the diagonally effective rays of the sun then the water in the cloud form is acted on by the cold of the northern regions and in consequence is caused to precipitate in the form of water and snow. After being precipitated in the northern regions it is further acted on by the cold causing portions of the water to solidify in the form of ice. This process continues during the period that the sun is situated south of the equator until the equilibrium of the planet becomes disturbed by the piling up of great bodies of frozen water at one end of the globular body.

The great amount of matter carried to the north during this period causes the earth to shift its position with regard to the sun. That is, it causes the earth to gradually lean as each load of water becomes added in the solidifying form to the amount already deposited by each prior precipitation. This leaning process comes about gradually in accordance with the amount of matter detached from the southern regions and caused to precipitate in the northern regions by the exposure to the action of the cold.

During this same period, while the water is being dispersed from the southern seas and carried off to the north by the propelling rays of the sun to again be pre- 


\section{VOYAGE OF THE EARTH.}

cipitated by the action of cold, the earth sinks nearer to the sun owing to the sun's heat that would otherwise be utilized in driving the earth away being transformed into its equivalent of work. The sun's driving power being transformed into an equivalent of work as a transporting agency, carrying the dispersed matter from south to north; consequently the earth must of necessity sink nearer to the sun during this period of the year.

The varying winds during this period of the year in the north are brought about by the diagonally effective power of the sun's rays with regard to this particular portion of the planet. The rays being effective only diagonally then it is nothing more than natural that the cold from transverse directions would bear in on the lines of force generated by the propelling rays.

During this period of the year the sun bearing along the line of the equator aided by the cooling action in the absence of the sun shining carry on the revolution of the earth on its axis as before mentioned in this work.

When the earth has leaned so as to expose a great deal of land owing to carrying great weight in the north then the sun becomes effective on the land of northern regions as the earth continues to sink while being driven diagonally by the action of the rays of propulsion upon the land. The sun acting on the land begins to drive the body as a whole in a diagonally effective way, while yet the planet sinks toward the south, owing to not having the sun acting directly against the 


\section{HEAT AND COLD.}

land as a whole. And continues to sink until the sun becomes effective upon all the land in the northern hemisphere by having settled so as to leave the equator south of the sun. Then the sun bears so heavily upon the solid bodies of land, owing to there being such great amount of land in the northern hemisphere, that it forces the planet as a whole away from the sun. There being no great amount of water in the northern hemisphere and in consequence the body is driven away a great distance from the sun during this period of the year. Land being of a solid consistency and not being easily detached by the dispersing rays of the sun, and in consequence the heat buoying must be effective on the planet as a whole.

During this period of the year the water that becomes dispersed from the northern regions becomes carried off to the south by the rays of the sun, and when south it becomes acted on by the cold and caused to precipitate in the form of rain and snow. Each precipitation adding to the solidifying process going on in those regions owing to the loss of sun power. The ocean currents also carrying great streams of water into the southern regions, which become solidified by the extremes of cold. Cold being extreme in this portion of the planet, owing to the planet being farther from the sun during this period and also denied the warming rays of same.

As the currents from the north continue to bring in water that becomes solidified, in addition to the precipitation in the form of rain and snow, it causes the 


\section{VOYAGE OF THE EARTH.}

southern portion of the planet to become heavy with regard to the northern portion, and in consequence causes a leaning of the body as a whole. Which leaning continues in conformity with the amount of matter deposited in the solidifying process. This process causes the planet to lean so as to cause the equator to change with regard to the sun.

The equator having changed with regard to the sun causes the sun's power to act on the body, forcing the sun to act on the frozen mountains of matter in the south. And while the sun is utilizing its dispersing rays upon the frozen waters, transforming the dispersing rays into an equivalent of work, the planet continues to sink toward the north again. Thereby the powers of the sun are again transformed into work while the earth works off toward the north to again take up the northern routine as before.

At the time of the year that the sun's power is being utilized in the melting of the southern solid bodies of frozen matter accumulated in the southern latitudes, we begin to have autumn season in the northern hemisphere. The melting occasioned by the accumulated matter causes the planet to sink nearer to the north until the power of the sun becomes active again on the waters of the southern oceans and the period of winter sets in in the northern hemisphere.

The change of climate occasioned by the shifting of the planet from these causes has a very material effect on all forms of life as well as all mineral and material substances. The change in the relative 


\section{HEAT AND COLD.}

equilibrium of the forces of heat and cold during this shifting process goes far in proving that heat not only aided by cold carries on these processes but dictates to life of all forms its course during the change in the relative equilibrium of the forces.

During the period of spring, or the period which brings about the shifting of the planet when at its northern extremes so as to expose a greater portion of the land to the action of heat, there becomes a period of expansion in all earthly substances, through the action of heat. The expansion is not only applied to the vegetable life and water and mineral, but is also found to be present in the domains of the animal life, including that of man. In every form of matter and animal and vegetable life the expanding powers of the sun become manifest during this period of the year, and continue throughout the summer season. Again adding another link in the chain tending to prove that heat tends to expansion or dispersal.

During the period of the year when the earth begins to shift from the south so as to move toward the north, being brought about by cold, there again appears in the northern portion of the earth a cessation of the expansion of earthly properties. This cessation does not apply to vegetable life and mineral products alone, but to all animal life as well. This cessation of the expanding movement continues on into the winter and up to the time the earth takes a shift to again get a greater amount of heat on the portion of the earth in question. 


\section{VOYAGE OF THE EARTH.}

These questions go far toward proving that cold as applied to material substances carries out the same law throughout the animal kingdom, as well as the vegetable kingdom. These points tend to show that cold tends to cohesiveness when applied to animal and vegetable life as well as to all material substances. These points tend to prove that heat tends to expand no matter how or where applied.

The material property in no matter what form, when subjected to the action of heat, will expand. A material property in no matter what form will tend to congeal or remain within itself when subjected to the action of cold.

The desire in the animal form of life to mate is nothing more than chemical affinity brought about by the condition of temperature upon the material products that make up the different individuals. One being a positive and the other being a negative, and heat being present sufficient to cause one property of matter to have affinity for the other in the given temperature.

Just as heat and cold act as the dictators of the conditions of matter in any other form, they also act as dictators in the generation of life by bringing about a condition between the two that will admit of the chemical affinity in the given case.

It is to be hoped that the reader of the foregoing will resort to inquiry, by reading the scientific description of the movements of the earth around the sun, as accepted today, which reads very near as follows; of 


\section{HEAT AND COLD.}

course not in identical words, as this is written from memory:

"There was a time in the distant past when our world was a wanderer in the heavens, without law or order. It fell through space and finally into the ellipse around the sun. As it fell to the point of perihelion or nearest point to the sun, it was attracted by the sun's attraction. It tending to go off at a tangent and attracted at the same time, chose the course around toward the north in the ellipse. The power of mathematics being so great as to render it plausible that the course would be toward the point of aphelion. This process continues throughout the ages."

Now, while the author will not vouch for the words being identical for the description of the earth marking its course around the sun, yet he will vouch for the words carrying about the sense of scientific description of the phenomenon. It is a shame that children should have such nonsense pelted into their expanding brains during the schooling days. Such nonsense tends to lead the child to think and act through life along the same line of thought as was instilled within the expanding brain. A style of thought that is more ridiculous than the old form of supernatural. Tending to give mathematics the power of the former god, or powers to work miracles.

As a matter of fact, mathematics have no power, only to carry out the base. Add to the base by adding, multiply the base by multiplying, subtract from the base by subtraction, divide the base by division. If the 


\section{VOYAGE OF THE EARTH.}

base is wrong, the multiplication tends to multiply the wrong, and will bring about the most ridiculous results. If the base is wrong the dividing of same tends to mitigate the wrong. If the base is wrong, adding to same adds to the wrong. But if the base is wrong and we resort to subtraction, we get the results; by taking one base from one base and leaving nothing. Then going right in and hunting the right base to replace it with, and starting over again with the new base and building our foundation on same. In that way we get the results. But mathematics will carry out any base we apply to; when absurd will make the absurd more so. 


\section{CHAPTER V}

\section{OCEAN CURRENTS.}

The ocean currents, as observed all around the world, tend to prove the minutest observations in the powers displayed by the heat and cold on the properties of matter. Ocean currents prove the propelling power of heat, and the condensing power of cold on the properties of matter. Ocean currents prove that heat tends to disperse matter and that cold tends to union of matter.

Ocean currents prove conclusively that the propelling power of heat, aided by the congealing power of cold, cause the earth to revolve on its axis through action on the plastic properties of matter. A study of the course of the ocean currents have proven that they flow easterly all around the world, and are not deflected from that course, only so far as affected by the coast line of the continents against which they impinge. Of course there is some variation brought about in the course by the action of the cold in the north and south through the cooling action of cold upon the particles of water, tending to union. And a further variation brought about by the tendency of water to expand when below a certain temperature. Also by impact against the solid ice banks.

However, the general trend of the waters of the 


\section{OCEAN CURRENTS.}

globe tends to conform with the action of the sun in expanding the properties of matter, aided by cold in congealing the properties of matter in the eastern portion of the earth.

The cold of the north and south polar regions tends to regulate the equilibrium of the planet by the tendency to union of matter both north and south, through the action of cold on the properties of matter. The expanding qualities of water below a certain degree is an additional safeguard in guaranteeing the equilibrium by deterring extremes in any direction.

The destruction of the cooling zone either north or south would render our planet a wanderer without law or order. The continual rush of the elastic properties of matter to one end of the planet would destroy the equilibrium of the body and cause it to keep on flopping from end to end. It would have two forms of revolution, one from east to west and another from north to south. The revolution from north to south would cause extremes of climate of short duration and would in consequence destroy all life on the body.

Life, if any, would be very short lived, as it would require but a short time to cause the earth to change ends by the continuous flow of water from one end to the other, thereby destroying the equilibrium and causing it to tumble from end to end.

But as it would require two suns to cause one of the zones of cold to be destroyed, and in consequence we need not worry about the end for end tumble. Our sun cannot act along the line from east to west and at 


\section{HEAT AND COLD.}

the same time act from south to north, so we will not be likely to have any trouble in tumbling from north to south or vice versa. It requires heat to disperse the frozen properties in each of these zones, and as long as the heat is not forthcoming there is no danger of a change in the zones.

Now we will consider how the sun, aided by cold, causes the ocean currents as an accessory to the revolution of the earth on its axis. The sun acting with its repelling force easterly, causes the water to impinge against the adjacent continent, or coast line of same. Then the waters become deflected by the outlines of the continents in conformity with the general trend of same. When deflected it becomes aided by the cooling action of the zones of cold in which it has been forced by the original power of movement. The water entering a zone where the tendency of the water is in the range of cohesion, it in consequence must be caused to move faster until the margin of expansion has been reached in the body. When the water has been deflected south by continental outlines it is aided by the cooling action of the cold until the point of expansion has been reached in the body of water. The excess of flow that continues beyond the margin of the expanding in the southern waters becomes deflected from the southern ice banks and is further aided by the propelling power of the sun easterly until again affected by the continental outlines, against which it again impinges.

Let us take observations on the action of the sun 


\section{OCEAN CURRENTS.}

in forming the current in the Pacific on the eastern coast of China.

The sun bearing easterly causes the waters to impinge against the continental outlines of the Americas. Such waters as impinge against North America will be deflected southerly, owing to continental outlines, barring a small portion of reflex waters which make off to the north. Waters that impinge against the coast of Central America take a whirling movement, owing to continental outlines. The whirling movement continues until the waters are again taken up by the general froce of the sun bearing easterly, aided by the greater portion of the deflected waters moving south through continental outlines aiding same. The waters that impinge against the South American continent are deflected south, owing to continental outlines, and join in the general flow from the north.

All these waters moving to the south are aided by the cooling action of cold on the properties of water as long as the limit of expansion has not been reached in the waters. The cooling action being a tendency to union of matter and in consequence the waters gain speed in proportion to the tendency to union in the particles. When the point of expansion has been reached in the waters they again become deflected by impact toward the northeast, to again become influenced by the action of the sun in the given latitudes. Then the waters continue eastward until by impact against the continent of Africa, and its consequent outlines, the waters are forced back toward Central America, to 


\section{HEAT AND COLD.}

again be deflected by continental outlines easterly. And again become aided by the sun in their course on toward Europe.

Waters deflected by the southern portion of the African continent are forced off southerly and by impact against the southern solids are again forced toward the east and aided by the propelling power of the sun and aided by cold within the range of union in temperature until they join the waters at the place of beginning. Waters of the reflex order all around the earth are again taken up by the general currents working toward the east.

The impact of the waters against the continents aids in moving the planet on its axis. They are no mean force when considered as a whole upon the land bodies of the different continents against which they impinge. The greater lifts toward revolution being manifest in portions of the continents like Central America, Africa and Europe where the waters are forced back toward the source of power or forced to take a whirling motion to again be taken up by the general currents around the earth.

In summing up the general course of the ocean currents they add greatly to the proof of the sun propelling and cold congealing the properties of matter in order to carry on the revolution of the planet on its axis.

The sun propelling when setting toward the west upon the waters of the ocean, while in the other ocean the cold, by its cohesive tendency, aids in causing the 


\section{OCEAN CURRENTS.}

waters to move easterly. And when the sun changes to the other ocean then the cold acts in the vacated ocean on the properties of water tending to cohesiveness toward the east. So the good work goes on eternally from day to day and year to year until the sun shall lose its power to propel. That meaning its extinction, as it will propel as long as it will exist. 


\section{CHAPTER VI}

OBSERVATIONS ON THE MOVEMENT OF THE TIDE GO FAR TOWARD ESTABLISHING BEYOND DOUBT THE POWER OF THE SUN IN PROPELLING; AND COLD AIDING SAME BY A TENDENCY TO UNION OF MATTER, IN CARRYING ON THE REVOLUTION OF THE EARTH ON ITS AXIS.

The tide is caused twice a day by the sun taking a lift on each ocean during the period of the day at which it leans to the west sufficiently to bring its power of propulsion to bear obliquely with regard to the planet. The sun sinking in the west so as to bring its rays to bear on the surface of the Atlantic. The Atlantic being a convex surface laid out against the sky where the sun is sinking down in the west, and the sun's rays being brought to bear obliquely so as to tend to lift the waters off at a tangent from the convex body. In consequence the sun lifts the waters off toward Europe. The waters necessitating a certain period of time after the lift to flow to the European continent, and the tide with regard to European regions in consequence.

Some hours after the setting of the sun in the At- 


\section{MOVEMENTS OF TIDE.}

lantic regions it arrives at the same location with regard to the Pacific ocean.

When the sun sinks down so as to bring its rays to bear on the convex surface of the Pacific ocean it lifts the waters off at a tangent. Tending to throw the waters off onto the American continents. The waters necessitating a period to flow toward the continent of America after the lift becomes at its maximum, and in consequence the period of high tide on the coast of America.

The maximum tide on the eastern coast of America is caused by the reflex flow of the lifted waters of the Atlantic, flowing back toward America after the sun has departed, and after the moving waters have flowed to the eastern continents and returned.

The maximum flow on the western coast of Europe being caused by the lift of the sun when sinking toward the west with regard to the Atlantic ocean; or the lift of the sun tangentially with regard to the convex surface of the Atlantic ocean.

The maximum flow on the western coast of America, or the Americas, is caused by the lift of the sun on the waters of the Pacific ocean, when sinking in the west. Or when taking a lift tangentially with regard to the exposed convex surface of the Pacific ocean, lifting the waters off toward America.

The maximum tide on the eastern coastline of Asia is caused by the reflex flow of the lifted waters of the Pacific after expending the rushing movement of the waters of the Pacific against the coastline of the 


\section{HEAT AND COLD.}

Americas. The reflex coming back onto the coast of Asia, and making the high tide with regard to Asia.

The secondary tide in all places is brought about by the second rush of the waters, aided by the congealing action of the cold upon the particles that make up the water. Sufficient time having elapsed in all localities by the time of the second rush of waters from coast lines to be plentifully effected by the cohesive tendency of water while yet within the degrees of cohesive temperatures.

The contraction of the particles of water in a great ocean being sufficient, along with a rushing motion from continental outlines, to make quite a tidal flow.

The change from ocean to ocean being about the time of the secondary tide also affects the flow of the secondary tide in all locations of the earth; there being about twelve hours between the extreme lift as applied to each ocean.

The cause of a difference in the time of tide each day can be attributed to the effect in the rising sun acting as a governor of the proceedings, acting for a time in opposition to the tendency of cold on the particles of water eastward. The sun being lifting in opposition to the flow of the waters generated by the action of the cold for a few hours. That few hours necessitates a delay in the time the sun shall overcome the tendency of the flowing waters in the easterly direction. And in consequence a delay in the tidal lift on the particular ocean. Also the shift of the earth in its orbit affects the tidal lift with regard to either ocean. 


\section{MOVEMENTS OF TIDE.}

Where there is planetary obstruction it also tends to vary the time and flow of tides. Planets tending to obstruct the power of the sun in buoying the water away or onward, and in consequence the waters tend to flow in opposite directions.

Where the planetary obstruction is sufficient to entirely deprive the sun of buoying onward the waters, then the waters tend to fall toward the sun, owing to the lack of a lifting power to detain the waters in place.

Sun spots tend to cause the waters to move toward the sun, owing to the rays of the sun being deterred by the obstructing spots on the buoyant power that tends to lift the waters.

Taken all around, the tides tend to prove the power of the sun in lifting or dispersing of matter as in all other properties where its power becomes manifest. The tides also tend to show that cold aids in the process of carrying on the movements of the tides and also carry the earth around on its axis.

The back flow also tending to, in part, delay the tides in the following twenty-four hours. The back flow necessitating a certain period of time to cover the given distance before it would come to rest. And in consequence the delay in the lift twenty-four hours later in overcoming the moving force in the opposing direction. 


\section{CHAPTER VII}

\section{EARTHQUAKES.}

Earthquakes are truly caused by the sun spots or planetary obstructions in the way of the propelling rays of the sun, that tend to carry on the revolution of the earth on its axis.

When a body of great size or dimensions passes over the face of the sun it takes away the rays of propulsion tending to carry the earth around on its axis, by obstructing the same. The sun spots or planetary obstruction, when affecting the earth locally where the earth is structurally firm, does not materially affect the earth. When the sun spots take away the propelling rays from over the waters it tends to make high waves and rough seas. When affecting portions of the earth where there is a fault in the geological structure, it tends to separation of that portion from the rest of the land along the line of where the power propelling has been cut off by the obstructing medium. Where the fault takes in a small portion of territory and the portion has been deprived of the lifting power, then the portion of territory tends to fall toward the source of lost power in its propulsion.

The fact of earthquakes occurring at night does not alter the case in the least, because there is a zone around the sun affected by the sun spots, and that par- 


\section{EARTHQUAKES.}

ticular zone bears upon a particular zone. or belt around the earth. In consequence, when the power propelling around a certain belt around the earth has been cut off, it tends to pull and drag on the belt around the surface of the earth. The quake always apparently dragging from one direction toward another tends to prove that it is the loss of the power in that quarter that causes the pulling toward the source of lost power of propulsion. The loss of a lifting power in a particular place will cause the subsidence in that particular direction where the lift is required, and that is where the pulling comes in in the case of the quake.

Observations have proven that the sun has a zone where the sun spots occur and in that zone only they are observed. The earth having a zone of earthquake susceptibility, backed up by not only modern observations but history of the past ages reveal the same susceptibilities. The fact of the zone on the sun tends to show that the zone has connection with a particular zone on the surface of the earth. Granting that it does, it adds to the proof that the sun lifts, or tends to separation,. and its power being denied in the premises, it causes a falling toward the place of denied power on the part of the particles of matter that make up the earth's surface.

In proof of a power being denied the earth in propelling in its revolution, we must get back to the actual daily observations and see what heat does when applied to matter of any sort. Does heat expand when applied to matter? Does it reduce particles of matter of all 


\section{HEAT AND COLD.}

descriptions to their constituent elements involving separation? Does it tend to make matter buoyant? If you can answer these questions in the affirmative, why not extend the powers infinitely? Why curb them at a distance?

If heat has these powers it certainly has the power to propel planets as well. And if it has the power to propel we will prove why the power has been obstructed in the case of the sun spots. The sun spots, ranging in a belt around the sun, and reason says that that particular belt has its powers centered upon a belt around the body it tends to propel. Being deprived of that propelling power on any portion of a belt around the surface of the earth will tend to cause a pulling in toward the loss of moving power in carrying on the revolution in that particular portion of the earth. Because that belt around the earth derives its moving power from the source being obstructed by the spots over the face of same.

The reason of great damage being done along the water front, where there is a geological fault, can be attributed to the fault first, and in addition to the fault, being bordered by water, which moves away toward the place of lost sustaining power. And in consequence, the portion of land being denied the sustaining power, moves toward the portion of country where the lifting power was cut off. 


\section{CHAPTER VIII}

CATACLYSMS-THEIR CAUSE AND EFFECT. GLACIAL EPOCH AND GEOLOGICAL CONFORMATION SURVEYED.

The earth had various changes in the climatical conditions which render evidence until today of past extremes. And flextures in the earth's crust tend to show a part of the earth's former experiences.

There have been various theories set up by men in explanation of the causes leading up to the freezing up of the earth's surface and the final return to normal conditions. Each lacking reasonable grounds for their acceptance as the truth in the face of the evidence revealed in the earth's crust.

The theory of the earth turning from end to end can not adequately apply in the case without giving reasons why the earth turned from end to end. Likewise we cannot accept the theory of an upheaval without the cause is set forth why, and the cause of the upheaval. Then it would be hard to accept it, even after giving a rational reason for the upheaval, in the face of the evidence remaining in the condition of the broken crust of the earth.

To consider the question in the face of the evidence accumulating every day beneath our gaze in the powers of heat and cold when applied to matter, we 


\section{HEAT AND COLD.}

arrive at the solution of the problem. In weighing the problem in this scale we need not give to heat or cold any powers other than that observed by the school boy on his way to and from school. Simply say that heat tends to separate, and that cold tends to unite. We need no other powers for heat and cold to explain every trial our planet had from the gaseous period to the present day.

Now we will weigh the earth in the light of the scale of heat and cold on the particles of matter, each exerting the power as observed by the school boy.

Our planet having cooled until all the waters and other elastic properties were frozen up. This cooling being brought about by the radiating of the heat into space. The properties of matter solidifying and in consequence, the reduction of the revolving power of the earth on its axis. Reduction of revolving power being a loss of power in propelling a foreign body that tended to move toward the earth. Along with the reduction of revolving power, the freezing' up of the elastic properties tended to reduce the reflecting power of the sun. Reduction of the reflecting power of the sun tended to reduce the power of reflection of a lifting force onto a body that tended to fall toward the earth.

In the face of the evidence, where had the earth any chance of deterring the coming of a foreign body from space onto the earth? If a foreign body had fell onto the earth, what would be the result? Would a foreign body have generated heat and reduced the frozen properties to air and water and other gaseous forms of 


\section{CATACLYSMS.}

materials? Would the foreign body have rendered the former beds of oceans mountain tops, by having driven another portion of the earth's crust inward, making a deep hole in which the waters would bed? Would the jolt from the foreign body have rendered portions of former forests so low as to go beneath the waves of the new ocean bed? Would the jolt from the foreign body calise the twisting of the greater portion of the earth's crust? Would the jolt of the foreign body bury large forests beneath the twisted surface of the earth?

It appears very reasonable that all these things could have come to pass by the infliction of the body from without. And it not only seems reasonable that it did occur, but it seems as if there is no way in nature to prevent it from occurring again whenever the earth shall have cooled so as not to admit of the sustaining of a body of weight in space. What is more, it is natural that it should have come to pass, and more than certain that the moon will add to the life of the earth in the same way, and millions of years later Mars will again add to the earth's life in the same way.

It is very likely that the body that was precipitated upon the earth was much nearer than the moon is at the present day at the time of its final collapse upon the earth. The earth losing the power of revolution, and along with revolution a loss of the power to reflect the rays that would buoy up the body would necessitate that the body would sink nearer to the earth.

It having sunk nearer to the earth until the power to withhold it was less than its weight in relativity. And 


\section{HEAT AND COLD.}

in consequence it would move toward the earth, and would be aided when it entered the remaining zone of what centripetal force the earth had left in revolving. But the centripetal force generated by the earth in the face of the conditions would be very little, owing to nothing being left of the elastic order in which cold and heat could act on conjointly. However, it would gain in speed as it continued toward the earth, owing to having no power to withhold it even though the centripetal force generated by the earth was at very near naught.

The heat would be sufficient generated by the impact to reduce the greater portion of the frozen waters to the gaseous form; and the greater part of the properties making up organic life would be reduced to the aerial form. To again be precipitated in the form of animal compounds whenever the reduction in the temperature became constant in the domains of germination.

It would destroy the greater portion of the life upon the planet, but not necessarily all, as portions of the earth may have escaped with a great shaking up. It would be determined altogether by the distance from where it had sunk to before it finally moved away toward the earth for the final jolt.

All classes of matter which were melted, or where heat was sufficient to cause their assuming the aerial form, were detained in space until the temperature of precipitation requisite to their forming compounds, or their temperature of precipitation in quality. 
If the temperature was sufficient in the inflicted jolt to render water gaseous, it remained so in that form until the cooling action brought about the temperature where it forms by combustion. And such portion as did not fall together in the combustive order took on the slower method of union throughout time which has elapsed since the collapse. All other properties reduced to the gaseous form were acted upon by the same laws as that governing water. Having arrived at a point where they united by combustion, they joined by that method, and from that period joined by the slower processes.

GEOLOGICAL CONDITIONS MANIFEST TODAY ON THE FACE OF THE EARTH, TEND TO PROVE THE IMPACT FROM SOME FOREIGN BODY, AT SOME NOT DISTANT PERIOD IN THE WORLD'S HISTORY.

When the surface fluids or the elastic properties become frozen up, sufficiently to solidify the outlines of a planet, it requires but additional cold to cause the precipitation of matter held in suspension in space. And the precipitation of additional matter will render the planet heavy in proportion to the gaseous nucleus, thereby causing it to lower nearer to the source of the least power of suspension.

That is the case with our moon to-day, and it is subject to the lessening of the force of the earth to withhold it from falling on the earth. Freezing up of 
the earth would cause it to fal! on the earth, but in the meantime it would lower much nearer before the final fall.

In conclusion, we find that everything in the geological structure of our earth tends to prove the truth of its having had that come to pass in the tumble of a body or small planet upon the surface in years gone by. And everything in the geological structure tends to prove that the coming to pass of the collision was the salvation of our earth. Because if it had not come to pass, the earth would have been the means of building up the fallen greatness of some other planet long before the present time, by having been precipitated upon some other body. Our planet having been very near the end of the journey when the lift came by the precipitation of a foreign body which gave it new life and vigor by the impact.

Now we will take a cursory view of the evidence rendered by geology in support of the earth having been jolted by a foreign body, instead of having had inward convulsions, or disturbance from within.

Geology proves that the earth was covered with ice or nearly so. The surface of the earth is broken and twisted throughout, or nearly so. There had been forests buried thousands of feet beneath the present surface of the earth.

Fish and animal remains are found imbedded on the tops of the highest mountains. Beds of forests are found beneath the present ocean beds, all tending to prove that the ocean did not cover the whole surface of 


\section{CATACLYSMS.}

the earth, as claimed by geologists. Also tending to prove that the inner convulsion did not sink forests beneath the present ocean beds. Also tending to prove that the inner forces did not bury forests thousands of feet beneath the surface of the earth.

But instead tending to prove that the impact from without first sunk the territorial outlines beneath the present surface of the waves by driving that portion of the earth deeper by the greater part of the foreign body jolting at that particular portion of the earth. Driving that portion inward causing the waters to change to the lower levels, and leaving the former beds of the ocean raised up into mountains above the waters, thereby leaving the former ocean beds as mountain tops. And thus accounting for the fish and animal remains in the strata, which were deposited there by the washing sands while the portions were ocean beds.

Also accounting for the forests that rest beneath the waves at the present time, which were driven down by the impact. Portions which were thoroughly melted by the extreme heat in the regions were deprived of animal remains, and belong to the class of earthly formation now known as the portion of the earth belonging to the azoic age.

Portions where the jolt was not sufficient to melt, but broke and twisted the surface, caused the flexures in the earth's crust. All tending to show that the earth was jolted by some heavy body from without, causing the parts of broken surface to fall in all shapes, edgewise and otherwise. 


\section{HEAT AND COLD.}

Also tending to show that the earth is formed around a gaseous center; or the impact would not have broken such great amount of its crust, but would have detached a portion instead if the body was solid.

The earth's history is neatly written in the folds of its crust, and in the voluminous library we can find the hour marks of the buried ages. All it requires is reason and deduction from the processes manifest in nature in carrying on its building and unbuilding as observed daily beneath the gaze of all mankind.

All the occurrences that mark the pages of the earth's volumes will surrender truth, when questioned in the language of heat and cold as observed daily by all mankind. It requires no savant, but instead simplicity and utter disregard for the abstract, while questioning. We need not grant to heat or cold any mysterious powers, but instead the simplest we observe in our daily observations, to get the results.

Heat and cold are the forces, and matter becomes plastic when subjected to their wiles.

We must bow to facts by observing the simplest things in nature, instead of groping off in the shadows, looking for a supernatural who reveals, or an effort of the imagination trying to account without law or order for the beginning of things.

Supernatural was a means born of ignorance of surroundings; where the party could not account for a phenomenon, it had to have some means of coming about, and consequently it had to have a maker, who was more powerful than the being making the inquiry. 


\section{CATACLYSMS.}

But by all means it would not do to have the being resemble any other than the inquirer, who was the greatest in sight, and in consequence he gave the great one his own likeness.

The style of reasoning giving to the sun and other bodies in the heavens powers diametrically opposite to the powers revealed on earth, is nothing more than the last gasp of a dying supernatural, grasping at a straw in the last struggle with the light of reason. It is the remaining atom left in the brains of the scientists of to-day, bred of centuries of superstition. While bringing back their reasonings to earth in some things, they still try and leave a little to conciliate the remaining atom of generations of superstitious training remaining within their brain formations. Tending to prove that it is a hard task to get superstition out of the human family when once it is instilled within the growing being. 


\section{CHAPTER IX}

\section{MATTER, CHANGE OF FORM, APPLICA- TION OF MEASURE.}

Matter is the primal base of all existing things, and of all ponderable conceptions heir to the human mind. Matter is the base of life, and without beginning and without end. In fact, it is life, varied by conditions only.

The same properties of matter act the same way at all times when subjected to the same conditions; that is to say, when the elements are in the same proportion in relativity and temperatures remaining the same.

Matter is, in fact, life, and different measures of its elements in relativity under different temperatures will cause different forms of its expression. But in no case can there be a particle of matter and death be its condition. No matter what the condition of temperature, let it be the acme of heat or the zero in absolute cold, still change is taking place in the particle of matter. Change and death are inconceivable to the human mind as one and the same thing; therefore change is life and death is not.

Let us consider the action of the common alarm clock, a mechanical contrivance constructed out of the properties of matter by the handiwork of man, and see 


\section{MATTER, MEASURE.}

what it will do when subjected to work up to the extremity of its powers.

The clock having a spring, and the spring being wound up, will admit of a certain amount of work is it not a form of life generated within the condition? The expiration of the condition means expiration of the form of work or life so applied.

When that clock is bordering the limit of the form of work or life so applied within the condition, it goes through the same convultions as the man does when bordering the limit of his existence in the condition of life applied to him as a man.

Man is a compcund of material properties born of a condition of temperature in the cooling of the planet, and a recurrence of the conditions will produce man again and again. In the case of the clock having a spring wound up to do a certain amount of work, produce the same condition by winding the spring and the same form of life will recur again and again. In either case, the forming of a man or bringing about the recurrence of the condition of life within the clock, they are one and the same. Subject the particles of matter that make up the man to the same conditions that brought about life in that form, and life will recur. Subject the particles of the clock to the same conditions that brought about the form of life and it will recur.

The life existing in the form of movement and action on the part of the clock by the winding of a spring is heir to the condition of compression brought 
about in the particles making up the spring. When compression so existing ceases to exist, then life so applied goes out of existence. Bring about the same conditions, and there will be a recurrence of the former expression within the same properties.

Subject any form of matter to the like conditions that brought about any particular form of life or expression and there will be a recurrence of the former expression.

Let us study the qualities displayed by the wagon, a mechanical contrivance built by man out of particles of matter. Load a wagon up to the limit of its capability to endure beneath the load, and it will begin making its troubles known by speech. Man not having put the tongue in the wagon with intent to have same used for to carry on a conversation, and in consequence does not give the wagon credit for speaking of its troubles.

The language of the wagon is brought about by little tongues in the wood work of the wagon imparting air against the wood work from which they are detached. The means of speaking is not unlike the means resorted to by man, who imparts air from his lungs against the roof of his mouth by the aid of a tongue of material properties which nature has provided him with. If you continue to load the wagon after it has made known its troubles by speech, then it will go through the same series of convulsion as the man does at the time of giving up the ghost, and it will give up the ghost also.

Perhaps man will say when considering the quali- 
ties displayed by the mechanical properties or contrivances built by him, that he gauged them to do a certain amount of work. We will grant that he did gauge them to do a certain amount of work, and in addition we will grant that he was correct in gauging. Then the real question has arisen owing to his gauging same and knowing how to gauge same. Was it a knowledge of another form of life he possessed? How did man find out how much work the horse could do? How did he find out how much work the dog could do? How did man find out how much work the man could do? How did he find out how much work he himself could do? Then granting that he found out all these points by studying the natures of the other forms of life. Still there is another question more momentous than either of the others. How did each of these animals find out how much they each could do in themselves? How did the dog find out how much he could do? How did the other man find out how much he could do? How did ine horse find out how much he could do?

Then last, but not least, how did that wagon find out how much it could do, and how was it that it notified man through speech that he was over-loading it, before he, as wise as he appears to be, found it out?

We may answer all the questions in a few words. Man found out how much work all kinds of animals could do through studying them in their habits and trials. He found out how much work the matter of all kinds was capable of doing through study of same, but was unwilling to attribute life to any of them. But 


\section{HEAT AND COLD.}

ds matter of fact man was studying forms of life all the while, but denying, the properties their true standing in the world of affairs.

The phenomenon of the wagon protesting in the case of being overloaded was brought about by the impartation of air against other particles of the structure from within by small tongues of the wood work, and was a correct measure of the trouble imparted to the atmospheric waves.

Man, when making a similar protest, when under duress or bordering the limit to exist, does so by imparting air from within against the roof of a cavity of variable dimensions within his face, and the air becomes forced out against the atmospheric waves in notification of the trouble of which it is a correct measure. Hence we find man when under extreme trials does not apply to the code of sounds making his troubles known, but instead acts like the wagon or other mechanical contrivance by imparting air in measure of the disturbance.

Man having a code of sounds applicable to his wants in measurable quantities capable of being interpreted by one of his kind, tends to show that he has a more thorough developed organism than other properties built by himself, but does not prove that he is in fact more alive in truth.

Man, in consequence of thousands of years of training and heredity, was led to believe that the same action and qualities, when displayed by any other animal or property, under all conditions was mere chance or instinct. It is not conceivable to the ego ignor- 


\section{MATTER, MEASURE.}

amus born of corrupt supcrstition, many times instilled within the youthful manifestation of ages of progenitors, that he is a compound of the particles of matter like all other forms of life organic and otherwise.

In many cases he is forced to acknowledge that there is a superior intelligence displayed by other forms of life. But he quietly and peacefully disposes of the complicated problem by adjudging the same to animal instinct. The qualities that are displayed by man that appear above the ordinary conception, take a place among the gods. In animals the same qualities, or perhaps greater ones, must be explainable by the instinct of the animal, in order to buttress up the fancied superiority of the ego, the great ego.

There is a fact that is unquestionable, that there is difference in the intelligence of different forms of life, all explainable in conformity with developed organism. Regardless of what the species may be collectively, there still remains difference in the personnel or individual of each kind.

But in no case can there be a particle of matter devoid of intelligence as it will accept or deny the advance of other particles, thereby proving conclusively, and beyond a reasonable doubt, a delicate regard for choice. It must be conceded by the most enlightened of mankind that the power to reject or to accept the advances of nther forms of matter, and do it unerringly and without mistake, requires a mode of reasoning. And yet that mode of reasoning is one of the attributes that must be conceded to every form of matter. 


\section{HEAT AND COLD.}

As no other form of animal life having a language but the man, so as to be understood by one of kind, there cannot be a more unjust decision arrived at by man. It must be attributed to man's ignorance along with a desire to hold himself above all forms of life having languages of their own.

Should the most enlightened of mankind of to-day be placed in contact with some man belonging to a tribe of whose language he had no knowledge, and at the same time be confronted with a cackling hen, he ceuld not for his life distinguish between the language of the man and the language of the hen. Yet he would immediately say that the man was speaking, not because he knew he was speaking in a language of measurable thoughts, but because he was a man. The only reason why the hen is not speaking is because she is not a man, and nothing has any right to speak until the ego gives permission, or until he teaches the animal to speak his language, no other animal having any right to have a language that is too deep for the wise one.

Yet, as a matter of fact, no man that is willing to do justice, and give credit where it is due, who has made a study of animals, will doubt that they and each of them have languages perfectly developed within their respective families. 


\section{MATTER, MEASURE.}

TIME IS A MODE OF MEASURE APPLIED TO MATTER, SHIFTING BY CHANGE OF TEMPERATURE IN RELATIVITY WITHIN THE ELEMENTS.

Matter, when subjected to measure, surrenders to two modes of measure; first time the most important, and second, bulk or size.

Time, when considered as an entity, must surrender its existence in measure of casual application of elements of matter in relativity.

It has no limit and no beginning, but is always shifting to the measure of the different particles of matter in relativity.

When time is applied to the measure of the duration of the compounds that form the human body, its limit is reached at the time of dissolution of the particles into the elements. When applied to the movements of the compound as an organism, then it loses its measure at the cesration of the condition ct action.

But when applied to getting a beginning out of nothing and final lapse to nothingness, then it loses its conception even in measure. It is impossible to delve to the depths of nothing and nothingness while scanning down the ages weighed in the balance of shifting measure. It is equally impossible to separate the particles which have been weighed from the unponderable nothingness while gazing into futurity: therefore, we must accept the measure when so applied to be forever.

When you can limit matter to a measure of duration regardless of change of form or destroy it, then you can attach time as the tombstone. 


\section{CHAPTER $X$}

\section{THE AGE OF WATER.}

When the earth had cooled so as to admit of its radiating heat conforming to seven hundred degrees of temperature within the greater portion of the gases held in suspension around the earth, then there was an explosion within the hydrogen and oxygen gases held in suspension, transforming same into steam. When transformed into steam, the gases expanded many times and in consequence were subjected to the extremes of cold by greater bulk. The deluge fell upon the earth and cooled the surface of the earth. When the deluge came into contact with the earth, it being'so much hotter than the temperature consistent with the detension of waters on same that there were many deluges caused by the escaping steam from the earth's surface, but each time the water was forced off into space by the heat it expanded so as to come into contact with the extremes of cold, and in consequence was precipitated back to earth. This process continued until the temperature of the earth was reduced so as to admit of the detention of the waters upon the earth.

The temperature being reduced below two hundred and twelve degrees of Fahrenheits thermometer, and in consequence a cessation of the deluges owing to the detention of the greater portion of the waters on 


\section{THE AGE OF WATER.}

the low surfaces of the earth, the escaping steam being held in suspension owing to being incapable of expanding so as to come into contact with sufficient cold to cause same to be precipitated.

In consequence, after the water had caused the crust of the earth to be reduced below the temperature of volatility, then the escaping steam or vapors from the ocean was not sufficient to reach the colder regions in order to cause same to be precipitated until there was a further reduction of temperature all over the earth. The further reduction was aided in being brought about by the condensing of mists on the surface in small quantities to again be driven back to the aerial world. This process continued throughout the period of the generation of life of all forms.

The worldly temperature being brought to an equal temperature by the waters during the period of the great deluges occasioned by the great expansion of the steam after each deluge. But the reduction below the temperature of great expansion occasioned a cessation of the period of the great deluges. And no further deluges occurred during the period of the generation of life of all forms. During this period of life the heavy mists hanging over the earth excluded the sun so as to render the cooling process thoroughly within action of an even distribution of the heavy mist which aided in the compounding process. The other properties held in suspension being assimilated by portions of the steam or vapors hanging above the surface of the earth.

The earth radiating heat to the gases and the cold 


\section{HEAT AND COLD.}

acting on the gases from without rendered the water supply all over the earth's surface, with the exception of the oceans, a matter of contact with the mists. The mists taking up the heat from the earth and imparting same to the cold of space. So the cooling process occasioned by the heavy wrapped mists about the earth from the period of last deluge until the cooling of high points was very slow. And the period of last deluge must have been very distant, when the high points of earth's surface became reduced in temperature so as to admit of the precipitation of water in the form of rain as we see to-day.

During the period when life was generated, the mists were heavy enough to guarantee all forms of life sufficient water through contact with same. The contact with the mists caused the pores to take up water and other properties which were precipitated with same upon the surface of the bodies. The mists were an aid in bringing about life by keeping the temperature constant; by secluding the action of the sun from direct contact with the waters. 'The mists hanging about the earth were partly transformed into the various compounds during this period. As this was the period of the greatest amount of compounds coming into being. Compounds taking oxygen and hydrogen from the compound of steam existing in the form of mists, and compounding same with other properties. The mists during the period of the generation of life also prevented the cold from acting on the surface of the earth, thereby guaranteeing even temperatures of constant, in con- 


\section{THE AGE OF WATER.}

formity with the elevation and means of radiation. The cold on the outer borders of the mists tending to condense same, and the heat from the earth tending to disperse same. In consequence the mists were held in suspension at a given distance from surface by the two forces. The heat of the oceans derived from the heat of the body of the earth tended to add to the mists, but the mists gave off portions of what was in space to the compounds, also to life of various forms after life come into being. The heated surface of the earth taking up some moisture and giving off in the cooling action also tended to offset the evaporation from the seas occasioned by earthly heat. And the sun could not act on the seas owing to the heavy hanging mists laden with the various properties going to make up organic matter. That is, the heat of the sun could not be brought to bear so as to disperse water from the surface of the seas. But its expanding action was manifest in opposition to the congealing action upon the mists and aerial properties in suspension. Its power was greater toward revolution, also owing to having such great bodies of matter in the aerial form capable of being affected by the moving power of same.

Its power was also manifest on the action of tides and ocean currents during this period by the pressure brought to bear against the aerial properties which had to press against the watery surfaces. The aerial pressure being greater than at the present time, owing to such great loads of material being held in suspension. 


\section{CHAPTER XI}

\section{VOLCANOES.}

Volcanoes are caused by the settling of the earth inward on the gaseous nucleus, owing to the increased weight of cooling crust. The settling of the crust inward causing friction in the particles of crust working against each other in settling. This friction causes heat and a tendency to dispersal of matter. Heat being motion tending to disperse, and in consequence must find an outlet, whenever it becomes manifest in excess of the power tending to offset it in the particular locality.

This friction being present near the surface where the lifting power of the heat is greater than cold in pressure in the premises, and in consequence it forces an outlet, thereby dispersing matter which has been reduced by the given quantity of heat. The heat having forced an outlet, must cease to continue whenever the settling of crust which generates same ceases to produce heat. It having no means of generating heat other than by the settling of the crust, and in consequence cannot continue longer than the means of reconstruction of action or movement, and the replenishing force being friction.

Where this friction is nearer to the center of the body, or nucleus, it will force an entrance inward 


\section{VOLCANOES.}

toward the nucleus, owing to least resistance, and thereby it will expend itself inward in adding to the heat of the central gaseous medium, or the gaseous centre which makes the earth buoyant so as to choose its line of action between heat and cold in revolving about the sun.

Volcanos are thereby caused by heat and cold acting upon the properties of matter, cold causing compression of crust, compression of crust generating friction within the particles of matter. Result, expansion or movement tending to dispersal, which is heat. Heat being greater than cold in the given locality, and in consequence liberation of same into space.

\section{CHAPTER XII}

ORGANIC LIFE IN THE BEGINNING, INCLUDING THE LIFE OF MANKIND, ALONG WITH FORMS OF ANIMAL LIFE.

The question of life in the beginning has always been considered beyond the power of man's conception in the solving, yet there is nothing more than a question of temperature in the cooling of the materials that make up a world, to be considered in rendering the problem very clear to us.

As the conception of life, considered in its natural 


\section{HEAT AND COLD.}

production, by the means we observe, will prove that a certain temperature is necessary in the commingling of the chemical properties in forming life of any kind. And in consequence, it becomes our study as to what time and condition in the elements would bring about the same conditions of temperatures in order to bring into play the same mineral elements in forming the compound. That is to say, our study will lead us, first, to inquire what temperature is necessary to the germination of the properties into a living mass, by observation in worldly production. Next question that confronts us is, can the properties be found in nature that go to make up the union or compound forming life? Our next question is to inquire of nature in the building process, whether the properties that go toward making up the living organism could be consistently and systematically brought together in conformity with their temperatures of precipitation within the given condition revealed in the germinating process, as revealed by the life producing process?

In answer to these questions, we find that the temperature was at one time present in the cooling process of the planet, necessary to the germinating of the particles of matter necessary to the formation of life were also present. We find that in the natural and unequivocal order of precipitation the chemical properties would all mingle within that given temperature. We also find that the period of constant temperature, or period of incubation necessary to any and all forms of life were a matter beyond all question of doubt present. 


\section{ORGANIC LIFE.}

Now, why should we doubt the means of the presence of life in the beginning on our little planet?

The most skeptical will readily admit that life now in the production by means we observe daily will have to await a given temperature. When the temperature has arrived, there must be certain chemical properties present which have a chemical affinity for each other. There must be a union of the chemical properties, and it must be within the given temperature. Then there must be a period of constant or nearly constant temperature of a lower grade than the temperature of union. This temperature being the period of incubation, or dormancy before the organism thoroughly develops into a knowing, breathing entity, or becomes cognizant of its life.

Can man be possibly so blunt of imaginative conception as to believe that every part of this process was not duplicated in the cooling of a great planet? Can man be possibly so blunt of conception as to believe in the face of all his knowledge of the properties of matter that the chemical properties were not in existence at that time?

All the properties that go to make up the human organism with the exception of the mineral properties range around the temperature of the germinating process, when considered in the line of precipitating of same in the building of the planet. Such properties as are of the mineral order in the make-up of the organism act as catalyzers within the given temperatures necessary to the germination of life, causing the other prop- 


\section{HEAT AND COLD.}

erties which are in suspension to unite in the given temperature and hence life. The mineral properties making up a portion of the organism having been precipitated at a greater temperature, but having chemical affinity for the other properties in suspension cause same to unite in the temperature of germination.

When the life giving temperature was present upon the face of the earth it became productive on different portions at the same time, owing to the revolution of the body in space, and the additional zones around the planet, to be further varied by the topographical conditions, periods remaining constant after germination in portion of the earth longer than others. And in consequence different forms of life. Places near the poles denied the action of the sun must have brought into existence the earliest form of life, owing to having arrived at the temperature in advance of other portions. Further, portions of the planet situated near the poles, or at the poles, must have brought into existence animal life of the order that has a short period of incubation, owing to having a short period of constant temperature in the cooling process.

At the poles, the earth had but one influence at work in the cooling process, and that being radiation of the heat into space; in consequence, the period of constant temperature after germination was short and life of that order in consequence.

Consequently, we find the dividing line between the species thoroughly defined in the cooling process in the same property; namely, protoplasm. The period of 


\section{ORGANIC LIFE.}

incubation, or period of a constant and life-building in the forming organism after the germinating of same marking the dividing line between the species. We also find portions of the earth's surface conforming to the period of incubation of every known form of species.

The variations from north to south making room for the dividing of periods of incubation. Revolution of the planet on its axis further varying the time or periods of incubation. Topographical conditions marking extra dividing lines between the species. All considered as to their action on the particles of matter yet in suspension, and further the action of properties already precipitated acting by chemical affinity on properties yet in suspension, causing union by combustion within one temperature to dwell within another. And the variations of time dwelling in a constant building process after germination marking the distinctive line between the species.

The change of the earth with regard to the sun in its revolution on its axis brought about a cooling of the properties held in suspension, and a corresponding temperature necessary to the union with properties already precipitated, caused the union of the properties from the air with the properties on the earth, and the germination of same into a living organism; to further develop according to the period of constant temperature where germinated. Consequently, the union was brought about by the contact of the properties in the aerial form during the revolution coming in contact with properties upon the 


\section{HEA'T AND COLD.}

surface of the earth which had a chemical affinity for them. It was a question of select or reject, as appears to be the law of matter until to-day. In fact, it is the law of the union of human choice in selecting a partner in life's process. Then the further constant occasioned by contact with the warm body of the earth after germination guaranteed a constant sufficient to form into some form of life during that cooling period. And life, of course, in conformity with the length of period of constant temperature guaranteed by place of germination. To be varied owing to zone or elevation in relativity with surrounding country.

A study of the basic protoplasm defies man's efforts in inquiry to distinguish any difference in the different forms of life, and in fact there is no difference. Only difference being formed in the period of constant after the germination of same into a living mass. Or the period of incubation. A further study of the germinating mass after its conception in any form will lend additional proof of the period of incubation marking the dividing line between the species. Because many forms of animal life are indistinguishable for a period after incubation; after the period has been reached a change comes to pass.

A study of the conditions of temperature taking place in the domains of inhibition of the different forms of life where the dividing line comes to pass will undoubtedly reveal to mankind the real cause of the change coming to pass.

World cooling is a very slow process, and it would 


\section{ORGANIC LIFE.}

be irrational for the man to believe that a period of temperature could be maintained in the body of an animal for one year that could not be duplicated in the cooling of a great planet. Especially when we have absolute proof of the earth having had every temperature from the burning gaseous form down to absolute zero.

The production of kind is circumscribed within a condition of temperature; disregard the temperature when at the right degree, and note the absence of replica in all forms of life. The chemical properties have affinity or will accept the advances of the other properties within the given temperature; and hence life.

Life was also varied in form and means of sustenance by the local surroundings wherein it came into existence for the first time. Life germinated upon the waters by the properties within the aqueous solution acting as catalyzers upon the particles yet in suspension when the period of temperature conducive to life came to pass; and in consequence, life of the watery regions, such as fish and other water animals. Of course the temperature of the waters in the locations where the germinating mass came into existence and the time of incubation varying the species.

Life generated along the junction of land and water where portions of the minerals from the land and portions from the water acted on properties in suspension causing them to unite and form a living, growing compound, and detained it in the inhibited form to 


\section{HEAT AND COLD.}

develop. It developed into the life known as the amphibian. Of course to be varied as to form by the period of incubation or period of temperature kept constant in the cooling process. Then a further variation was brought about in the developing being, owing to means of sustenance. It developing along the lines best conducive to its perpetuation within the conditions that were manifest in the surroundings.

Life coming into existence upon high precipices bordering the ocean, where there was very little means of sustenance within easy access, developed into the aquatic fowls owing to the inexorable law of condition. Of course they were varied by periods of incubation differing owing to time of constants in the locality. Also further variations were brought about by the chemical action beneath the surface in the precipitous regions when temperature was manifest in the cooling process, bringing into existence the lizards, snakes and rats and various denizens of like abodes. All conforming within condition of incubation and surface or subsurface chemical union within the germinating temperature.

Life of the order of snake and other burrowing animals were probably brought into existence beneath the surface after the surface became too cold to maintain a constant long enough to insure life of any order. The process being brought about by the leaching of material products within the earth until the chemical properties came into union within the temperature of union into life producing masses; to be kept constant 


\section{ORGANIC LIFE.}

for periods sufficient to insure life of the order of the burrowing animals. And it is very probable that life of the underground order is developing until today, beneath the earth's surface where heat is sufficient to insure a constant after chemical union sufficient to become manifest in the form of an organized life.

Life of the underground order having come into existence beneath the surface necessitates that it develop along the order of the means that brings into being life of the order. Or it develops along the order of condition as in the case of all surface animals.

Small insects being likely to have been the first form of life to develop on the face of the earth, owing to the highest points of minute dimensions cooling first, and their being small particles of land juting out above the balance of the land would necessitate but a few hours of constant temperatures. And in consequence, life of the ephemeral order, bugs and flies and small insects.

The waters having flowed for some time on the earth would have a tendency to cool off that portion of the land over which they flowed, and consequently would bring into existence a very early order of life. But it is very probable that the birds and fowls of different orders did precede the life of the deep. Because the high points would be to a certain extent affected in the cooling by the evaporation of the waters in the cooling of the oceans. The high points bringing into existence the birds of the air, owing to keeping a short period of constant temperatures through being exposed 


\section{HEAT AND COLD.}

to the cold of space being higher than all other lands. The bird life developing along the lines tending to perpetuation of the kind did of necessity develop a means of moving through the air with wings in search of food. Insects and other ephemeral life having preceded the winged life owing to coming into existence in portions of land juting up so as to keep but a few hours of constant temperatures. And consequently made food for the earliest winged fowls of any size.

Birds and winged life must have been the earliest form of life not only in northern regions but in all regions, owing to topographical conditions establishing conditions conducive to their existence in all climes in advance of other forms of life. High points cooling in all zones first. Not because the points were moulded higher in the building of the earth, but because the settling of matter in conformity with the different periods of precipitation would always choose the coolest part of surface in conformity with the laws of precipitation of each order of chemical properties. The points having been built by the choice of matter in the cooling, matter knowing but one law. Precipitation within the order of each property when the temperature of precipitation had arrived. Consequently the coolest point built higher than neighboring surfaces.

The birds and winged species having but a short period of incubation or period of constant temperature after the union of the chemical properties into a germinating mass. Consequently they conform to the 


\section{ORGANIC LIFE.}

high points in keeping a constant temperature after the union of the properties into a living mass. And undoubtedly followed next in order of life to the insect life, which was undoubtedly of the winged order also.

Geological conditions of today, studied in the light of geographical conditions, tend in a way to place the fish or life of the deep in advance of the winged life. But in the study of geological conditions as revealed today the question of a foreign jolt upsetting the surface of the earth must be taken into consideration in passing on the priority of life on the face of the earth. It must be remembered that the places where evidence of the order tending to place fish in the first order of life, are the former beds of the ocean before the ocean had a new bed made from the landed portions of the earth, by the jolt from the outside force. For that reason we can concede to that particular portion of the earth a priority of life in the order of water animals or fish. But we cannot concede to priority in the order of life when the land is considered along with the watery surroundings. Because the order of life in the mingling of properties into compounds of life, denies to fish the arrival of temperature consistent with the union of properties of matter into a living organism, in advance of the life of the landed portion of the earth prior to the jolt of the foreign body.

The high points had many reasons when once having arisen above the order of elevation of surroundings, for cooling faster than other portions of the earth. They 


\section{HEAT AND COLD.}

became exposed to the action of cold from the top and all sides working towards radiation. Each revolution tending to reduce them far in advance of all other surroundings. Each succeeding precipitation of properties of matter of a different order of precipitation necessitated an additional reduction of temperature, owing to increasing height above the order of surroundings. The additional reduction in the temperature occasioned by the additional elevation would necessitate another precipitation of matter of a lower order of precipitation. Thereby reducing the temperature of the mountain until the properties came within the order conducive to the precipitation of the properties, which would mingle into the germinating mass and develop into life. Following the condition arrived at where life could exist of the first order, additional reduction down the sides of the body of land forming the mountain would admit of other forms of life. On down and down the side of the mountain until to the lowlands there would be different periods of constant temperatures. Each to be regulated by the amount of land that would act as a warming body in the maintenance of a permanent temperature for a given time after the union of the life building properties.

As to the means of bringing about in each case the different temperature, along the line of union in the case of each life coming into being, and that being of a higher order, than the temperature consistent with incubation. Nothing is more easy of explanation. 


\section{ORGANIC LIFE.}

The earth revolving in space and surrounded at that time with gases of matter in suspension, each capable of precipitation within a given temperature whenever arrived at. The earth revolving with one side bordering cold of interstellar space where cold is equal in cohesive tendency to the action of heat from the other side of the earth where the sun drives or lifts on the same. During each revolution toward the cold of space the matter held in suspension bordering near the surface of the earth and being carried with it in the revolution, that would admit of precipitation within the given temperature being precipitated. When properties being held in suspension during this period of exposure to cold are of a chemical disposition that will admit of their action in union with other properties already precipitated. Then they unite. This chemical action between the property already on the earth and the property in suspension takes place at a temperature higher than the temperature of precipitation of the property held in suspension. The chemical action in the union of the properties first tends to raise the temperature, then finally settles down to be governed by temperature of the place of surroundings. Temperatures of surroundings when conducive to the maintenance of life, when the union is of properties capable of building up a form of life, and hence life.

Forms of life like the elephant and other animals of great bodily proportions were undoubtedly the product of lowlands, where there were great bodies of 


\section{HEAT AND COLD.}

the mineral products situated. These mineral properties acting on the properties in suspension when the proper temperature had arrived in like locations, and in consequence the precipitation of the life-producing properties into a germinating mass. The further fact of the lowlands hemmed in by surrounding highlands guarantee long periods in a constant temperature in the cooling process. And thereby further guarantee the long period of gestation, as is the case of the elephant or animals of that order. Thereby conforming to the period of constant in the production of the elephant, and adding extra proof of the advent of every form of life according to the order of maintaining a constant after the period of union of the properties. The further adaptation of the animal life of that order until today to like abodes lends additional proof of the means of his advent on the scene of life. He being adaptable to the conditions that were the means of his making, and in consequence will thrive in like abodes. Because the law of nature seems to be in all cases to build in conformity with the conditions which are to be the part of the form of life, and toward perpetuation within the conditions.

Variations in the elevations from the extreme lowlands and marshes up to the mountain tops tend to vary the different periods in which a constant temperature can be maintained by mother earth in the cooling process; and in consequence, life of practically every order within each locality. Of course, some parts of the country having properties in natural order of form- 


\section{ORGANIC LIFE.}

ation not adaptable to the sustenance of animal life of a particular order, and in consequence, absence of life of the order. Should life of the order have come into existence at the time of the building process in any given place where the properties in nature necessary to its maintenance are lacking, then it must have passed out of existence owing to lack of the nourishing requisites. Or more properly, the precipitation of properties into a germinating mass tending to form a certain class of life within the locality, could not develop into life owing to not having the nourishing requisites in the locality.

Temperature admitting of the condition of organic life of every order in the highlands in every locality first. And each succeeding form of life coming in the order along down the mountain sides in conformity with the period of constant necessary after the germination of the mass of protoplasm. Until its final climax in the production of the life of great bulk like the life of the elephant order in the extreme lowlands. Thereby marking difference in life of the first order.

Life of the second order commencing just below the surface, and as the earth continues to cool in conformity with the union of properties into life, life will continue. It is undoubtedly being produced today wherever there is the necessary temperature below the surface and where the mingling of properties can be brought about by the leaching process.

In all cases the metabolism, or feeding of the tissues in developing life during the early process, and during 


\section{HEAT AND COLD.}

the dormant period or period of gestation or incubation', was brought about by chemical affinity within the forming body as a chemical entity, and the chemical properties in suspension aided by the chemical properties within the properties surrounding it on the lap of mother earth. Or, in other words, the forming compound of life acted as a catalyzing entity on properties in aerial form necessary to its maintenance, until life becomes a fact by its living, moving and knowing of its existence. When completed into a moving organism it ceased to draw from the aerial form or earth in itself sufficient without additional food to maintain the organism, and additional action. And consequently became a feeder on other forms of life.

The new form of action and life occasioning growth and movement, and in consequence necessitated additional food to offset the increased work. In consequence, the new order of life of whatever order began to exact from other forms of life means of sustenence by feeding on same.

The mechanical process by which life developed after the union of the properties into a mass of protoplasm can be perhaps defined as a fermenting of the mass, owing to the long period of constant temperature after the union. Constant temperature tending to ferment in all cases. The ferments acting while fermenting upon the particles of matter in proximity and drawing sustenance from same. And acting as an entity on suspended properties necessary to maintain same. 


\section{ORGANIC LIFE.}

The dividing line between the oviparous and the viviparous animal kingdom can be conclusively attributed to the local conditions of the fermenting masses of protoplasmic matter. Where there had been but small bodies of protoplasmic matter thoroughly separated in different small lots, owing to the material substances that caused the particles yet in suspension to unite with them, thereby forming a small body which afterward was followed by the egg order of life or oviparous life. The small bodies of matter that acted on particles in șuspension not being sufficient to admit of more than one body of a given kind in a place. Thereby life of that order.

The life of the viviparous order being the product of large bodies of minerals acting upon particles in suspension and causing union of thousands of living organisms at the same time. They all being a growing in union with each other in the one large body of ferments. And in consequence, were necessary as a means of aiding life, owing to production by aiding each other in the order of first production.

Life of that order having had the aid of other life by contact in the beginning, must, owing to precedent in condition, need the contact of life of the same order as the means of earlier production, throughout all future production. The temperature remaining the same in cases of masses of fermenting protoplasm forming into life in a given lot of growing life; and in consequence, the life all of the same order when developed. 


\section{HEAT AND COLD.}

In the field of scientific research, so far as developed, it has held as a postulate that matter cannot be destroyed or that matter cannot be made. Accepting the truth of the indestructibility of matter as a fact, and the inability of man or conditions to make matter as an additional fact. Then taking into consideration the change in the temperature that has taken place in the formation of worlds until cooled off like our own. Then there seems nothing within reach of the mind which has occurred but what will admit of duplication within the conditions which had to transpire. In fact there is nothing within reach of the eye, aided by the most powerful lens constructed by man, or in fact, within the universe, but what will admit of proof within the laws of changing temperatures. There has never occurred for one moment or for one year any condition in the life of an animal but what can be duplicated in the cooling off of a planet. When the condition could not be found in one portion it certainly was to be had in another. And every property necessary for the construction could be had at the right time in one place or another during the cooling process. There being so many places upon the surface of the earth where the same conditions could become manifest, in case of failure in one there was always another to carry out the work. It being one more case where nature was not found wanting in many means of carrying out her work. And she had a million of safeguards in guaranteeing the carrying out of success in each case. 


\section{ORGANIC LIFE.}

Taking into consideration the many means of carrying the proper temperatures and union of matter within them that nature had in the cooling process, we find little trouble in finding the conditions that brought life into existence upon the earth. And we must acknowledge that life was as unavoidable in the conditions that existed at that time in the cooling process, as it would be to try to stop matter from union when the temperature has arrived for its precipitation.

With regard to evolution, there can not be any doubt as to time and trials bringing about change in the stature of the species. As the product of condition cannot subvert the law of its maker. Man and all animals, being product of condition, must submit to the dictates of further conditions. But as to evolution wiping out one form of species and transforming it into another, that is all rot. As the wiles of time leaves conditional marks on the youthful being, so does the conception of beings leave footprints throughout posterity.

As life is the product of a condition in the elements and temperature, then condition is its progenitor, and will tend to modify the original work by being itself modified, but not so as to change the form of same. The change being but adaptability to the conditions as they change.

But the idea of life being built up out of one minute form by evolution, and evolution being the maker of large bodies out of nothing but change, must be the worst of rot. It is strictly against the scientific truth of inability to build matter out of nothing. What 


\section{HEAT AND COLD.}

is evolution? Is it a supreme power that can build powerful beings out of nothing? Or must we attribute the arrival at the solution by that means as one of the last efforts of the supernatural atom in the mind of its originator to hold in abeyance the light of reason that was spreading within the brain of man? In which case the atom won out. Defeating reason by overpowering the reason that was coming to the man.

Instead of building, evolution has had a tendency to modify the proportions of the animal life throughout all the different families. And in return for such reduction in proportions it has tended to compensate all of them by greater durability owing to the conditions necessitating same in order to exist.

Wherever life has been built up, it has occurred by the means of breeding of animals within modified conditions. It has been brought about by a modification of the conditions by one of two means. First, by stabling the stock and preventing the rigors of climate. And second, by feeding the stock other properties of food which are not procurable in the order of nature. Being the product of the handiwork of man in cultivation. In all other cases it will be found that the tendency of nature is to diminish the bulk of body, of any and all forms of life, compensating them with a more durable constitution adaptable to the conditions.

The reason of reduction of body being in order that the means of sustenance within the order of nature may be sufficient to keep up warmth within the body of a. given size, a larger body necessitating a greater 


\section{ORGANIC LIFE.}

amount of food to generate heat to so much more body. A lessening of body will lessen the amount of food necessary to keep up a certain amount of heat.

The conditions being changed by man with regard to the stabled stock, and a further change being brought about by man in the order of food for such animals. Consequently, greater body is the result. Yet if the conditions were brought about by any other agency beside that of man, the result would be the same. Because it is not the Godlines of man that increase the bulk of the body of his stock. But instead, it is the adaptability of the animal to the conditions that he must live under. Conditions being the cause of the greater bulk. But the order of nature being to cool, the order of animal life in general, when not modified by some other agency, is to reduce bulk of body to conform with the order of nature. And throughout the past the order has been the same always to lessen the bulk to conform to the cooling conditions.

A study of the geological conditions during the Carboniferous age, as revealed by geologv. will conform in showing how animal life has deteriorated in the time that has elapsed since that period. At that time, there being winged life of proportions equal to the greatest of our land animals of today. They but conforming to the heated conditions of their age and time.

As to monkeys, apes, bears and other forms of life that resemble man, they each were bordering like con- 


\section{HEAT AND COLD.}

ditions to the conditions that brought forth the man, but the difference was plenty to divide the species into different families. Perhaps that a study of the temperature at the time of germination as observed today will distinctly divide for once and all time the relationship of the species, making it very clear what was the real difference.

As to one class of animal being transformed into another by time and change seems too foolish to consider. But where the periods of gestation are alike, or very near so, it is possible that a cross may be brought about between two forms of species. And the offspring taking on portions of the qualities of each of the animal forms.

Trees and herbs of all classes, and in fact all vegetable life, along with all cereal growths, were effected by the cooling process of the planet, just like the animal kingdom. The difference in elevation bringing into existence the order of life adaptable to existence at the greatest temperatute, and sc on down the orde: of each succeeding plant in the order of its relativity to the first in temperature. Each plant coming into existence whenever the temperature was present that would admit of its union into a growing form. And so on down to the order of the plant life now growing in the ice banks or snow banks in our coldest regions. Each coming within the earliest condition that would admit of same. 


\section{CHAPTER XIII}

\section{ORGANIC LIFE AND PLANETARY}

\section{CONSERVATION.}

Organic life becomes a great factor in the preservation of the planet, and adds perhaps millions of years to its period of duration. Vegetable growth and trees, herbs, cereals and all other forms of life of that order become a means of storing heat to again be liberated by the action of the digestive organs of animal life in transforming them into active bodily heat.

Among the animal and vegetable kingdoms there is no other form of life that adds to the period of the earth's duration as much as man. But instead of man being the one and only, and all earth being made for his playground, and all animal life to subserve his wants while at play, he is nature's greatest slave in the perpetuation of the whole means of life. $\mathrm{He}$ is the minor being moving toward the greater cause. Instead of the greater cause moving to subserve his immediate wants and pleasures. 


\section{CHAPTER XIV}

\section{MAN'S PLACE IN THE PERPETUATION OF THE PLANET.}

$\mathrm{He}$ is one of nature's greatest restorers of the dormant energies held in reserve, through his liberating same. Man generally believes when he makes some important discovery within the order of nature, by his studies, that he is revolutionizing the order of nature, in liberating the energy hidden away by nature. He gives himself the credit of being a more important being than the universe, being gifted with such great powers that to him all earthly matter and all earthly movement must bend in worship. He believes that all these great things were made by some great man, and that the greatness of the original being was natural, being that the great one who did the great work resembled himself. The being could not be other than powerful who should resemble the great one in himself, being of his image and likeness would in itself make the great one much greater. Man, in attributing all the greatness to one of like image and likeness, seems to be unaware that it is not the love of a great one, but is in fact the love of the ego coming out in the case of a third person. Every man who bows to such worship of the great one, in his own heart knows that the ground whereon the great one has been built; has 


\section{MAN'S PLACE.}

been purely and simply love of self. That is, if the man is of a disposition to think for himself, and not accept the rules laid down by others who do the thinking for him.

When the love of ego has been developed for thousands of years in the progenitors, it is hard for the man to conceive of a greater being or form of being than the ego. It is the love of self, pure and simple, taking the form of a distant one, for modesty's sake.

Man, instead of being the ruler of the universe and the maker of worlds, is instead its greatest slave in the order of nature, he being a necessity in the perpetuation of the planet. He is dictated to by the order of nature, and he must obey. Nature molded him out of particles of earth that he may work without intermission throughout his life, in keeping the earth inhabitable to his kind.

Man's greatest needs are manifest along the lines conducive to the perpetuation of the planet. He being one of nature's products and being made in the cooling order of nature to further conserve the working order of the whole body. Nature having molded him as all other forms of life. Molding each for a purpose. The principal purpose, and in fact only purpose, was the planetary conservation. Each form of life tending to readjust active heat upon the body of the planet, to further prolong its duration as a body.

Man's nature conforming to the purpose he was born to fulfill, he naturally becomes adapted to living and necessary wants along the line conducive to the 


\section{HEAT AND COLD.}

end. In that case, a man's necessary wants appeal to his loosening up of the dormant energies, of which the earth has great stores. Man dare not disobey, as his welfare, present and future, depends on that mode of action. He must work to that end or suffer and die.

Man's advent upon the earth being one of nature's means of continuation of the life of the planet. $\mathrm{He}$ was ordained through slavery to work without intermission in the good work of adding to the life of the earth. Necessity was the means of coercion, and necessity was always in the order of nature's requirements, at the particular time when the earth needed the liberation of extra heat to offset the cooling process within the whole.

Nature having built or molded man for a purpose, to aid in worldly perpetuation. And in consequence has many means of coercion within the kind when failure to comply with the great purpose has been resorted to. In the case of individuals deeming it better to evade their share in worldly conservation, nature has seen fit to deprive them of health, which is an attribute of the process which man was born to enjoy when complying with the means of his nature. He being born to a purpose, thrives within the purpose that molded him.

Nature finding the material that tends to make up his body too valuable to be wasted in a being of no energy, when the man has no energy to comply with the means of conservation. And in consequence it is nature's law that he shall wither and die, to further 


\section{MAN'S PLAACE.}

transform the material into an entity of value in conservation. Nature not desiring a waste in any of its means of its perpetuation of the body of the planet.

Man's greatest inventions are of the kind that transform great amounts of heat into the active form. The transforming of latent heat into active heat adds to the life of the earth. Great activity anywhere on the face of the earth has been observed to cause an extra precipitation of rain in like localities. Extra precipitation of rains in localities which formerly were dry tend to show that the temperature has been raised from a worldly standpoint, admitting of the dispersal of greater amounts of water from the seas, and consequent carriage to more distant points before cold would overcome the total volume, causing same to be precipitated. Thereby adding to the proof in favor of establishing man's place in the world's conservation. Also tending to prove that man has to conform to a certain amount of work in order that the portions of the earth may conform to his mode of life. His mode of life necessitating certain conditions that are procurable by the work, all of which are a necessity to the perpetuation of the earth, or prolongation of its period of duration. And all, further, tending to prove that man is the slave of nature by having to conform to the perpetuation of self in order that he may conform to the greater cause of worldly conservation. He being but the minor property aiding in a greater cause.

Every great discovery made in the studies of man, tending to transform great stores of latent heat into the 


\section{HEAT AND COLD.}

active form, tends to lessen the trials of man in keeping the earth inhabitable to his kind. And also lessening his graft in bodily labors. Thereby forcing man in every conceivable way to use his best energies to the carrying out of the good work of conservation. Thereby showing how man was in the beginning formed for a cause and acted and will act along the lines of the particular cause, as long as he can possibly maintain conditions adaptable to his existence. Where he does the greatest work toward the cause, he reaps the greatest benefit for himself and his kind.

All other forms of life are aiding toward planetary conservation by means of their digestive organs, which transform latent heat into active heat. But man is the greatest slave of the world, because he not only uses his digestive organs in transforming, but also the muscles of his body and every nerve cell of his brain. Man has also to act as the scavenger for all animal life, because he has to hunt up means of procuring food for all animal life; also care for their hygienic conditions as a further means of his own perpetuation. He is the servant of all animal life. He is the slave of all animal life. And, in final conclusion, we must admit that man is the one and only slave that actually exists. The slave of all orders of life as a further means of his own sustenance, to finally conserve the one great cause-planetary continuation of life.

To be more explicit in the judgment of man, in the face of his own reasonings, accepted worldly. His order of reasoning, as to the world and every conceiv- 


\section{MAN'S PLACE.}

able thing in nature being made as an accessory to his personal welfare. And being a secondary consideration with regard to himself. Such mode of reasoning can well be attributed to the order of reasoner, who grants to the sun powers off in the distance, diametrically opposite to the powers manifest at his finger tips from birth until death, throughout thousands of years of the coming and shifting of his kind. Always looking off in the distance for a solution of problems written in indelible characters on his body or on everything his digits touch. Looking off into space trying to make eligible, characters written by some unseen hand, of like resemblance to the one he is carrying. Seeking revelations from the mists of buried ignorance in the form of imaginative beings which have no existence beyond the diseased condition of a brain born of thousands of years of superstition.

There is no power in the buried ego other than the stench arising above the remains of last resting place, until final disintegration of the particles, which then may be transformed into some useful means of further force tending to carry on the good cause of worldly conservation.

The saying attributed to the great Confucius is very true when you come to know nature's law. The saying follows: "What the superior man seeks is in himself ; what the small man seeks is in others." Now when you know the law of nature or your maker you are quite convinced that the white man is forcing ignorance on the wise Chinese when he attempts to con- 


\section{HEAT AND COLD.}

vert him, to seek in others instead of seeking within himself. It is simply ignorance trying to dominate knowledge. The truth which the Chinese has been in possession of has led the Chinese to avoid taking from others what he knew to belong to others. He not having the precedent set for him to steal and lay in wait for a passing stranger so as to rob or kill him from ambush; in consequence, the Chinese has tried to live peaceably, and to do unto others as he wishes to be done by. The white man, in contradistinction to the Chinese, has had the precedent set by his god to look to others, both for knowledge and everything he desires in life. His god has taught him not to look for anything within himself. His god also lays behind a wall of seclusion and establishes precedent for him to follow in waylaying his fellowman. In consequence, the white man looks for everything he eats from others. His god teaches him to trust in others, in fact, forces him to obey others. His god establishes precedent in every wrong he tends to practice.

In consequence of the precedent and teachings of his god he has become aggressive. He is not satisfied with what he can make or get within himself. He makes war on the other fellow with intent to rob him; if necessary he must exterminate the other fellow so as to get what he needs. He has no right to think or act without disobeying and in consequence he has the order to convert the other fellow, given to him by his god. The converting of the other fellow becomes his trade in making war for gain, because he cannot work 


\section{MAN'S PLACE.}

to any end without eating and his god has given him no food, only what he can procure from the man he tends to convert. In consequence it becomes his godgiven right to rob, because he could not carry out the law of god without eating. In eating he robs, but he has the grace of his god backing the robbery in saving the other man from eating the food. If the other man resists the offense or desire to feed on him then he is killed with the grace of god.

The greater part of the Chinese are followers of Confucius, and in consequence believe in depending on the man within himself for means of sustenence. What is the result? Answer: A peaceable people, not aggressive, bearing a small place in history ripe with murder for gain by ambush or other means. The white man having ambushing precedent and a robbing precedent set by their gods, and in consequence has history very ripe with ambush and other foul means of murder for robbery. The white man is the product of the precedent set for him. Now he is not satisfied with having the greater portion of the world peaceable, owing to peaceable precedent, but he wants to carry his ambushing precedent in to the domains of the peaceable people of the earth. His reason being to carry out the robbery which his god has instructed. He cares not for the souls of his victims, but he cares for the gain he can get by following the precedent and command of his god. 


\section{CHAPTER XV}

\section{THE ADVENT OF MAN AND HIS DEVELOPMENT.}

Man came into existence on the continents in the middle highlands, on account of the conditions of cooling temperature of like abodes conforming to the period of gestation in that style of life. The middle course between high and low surfaces of the earth conforms to the period in the case of the man's advent upon the earth. And his adaptability as shown today proves that he was the product of equa-distant extremes in each order of earthly temperatures. And in consequence, adaptability to all.

The topographical conditions that were productive of the order of life that is the man's, has a great deal to do with the order of life that is his. He coming into existence in locations of earth surface where the country was level, or so near so that there were even temperatures of long duration. And in consequence, little else but the order of life he represented for long distances of earth surface. The mineral products necessary to the building up of life being distributed in like localities to admit of the building up of life. But no means of sustenance other than the products derived from nature in the suspended form 


\section{THE ADVENT OF MAN.}

in atmospheric order, along with the necessary mineral properties which were precipitated at higher temperatures, or which preceded his formation into a living organism.

The temperature having arrived in the cooling process which would admit of the precipitation of the basic compound at the foundation of life of all orders within this high level surface of earth, where the minerals conducive to life were precipitated earlier in the cooling process. This basic compound being protoplasm, and being plastic in the trials of conditions in which it is subjected to, added to the mineral substances into which it comes in contact with.

In the case of man, the protoplasm being precipitated in high or medium abodes of equal elevation or near so. There being iron and lime and the various salts going toward building up life within the locality before the precipitation of the basic property. The basic property being a product of nature that ferments and draws nutriment from the abodes in which it is detained, providing that a temperature can be maintained for sufficient time. A certain period of time being necessary for it to arrive at a condition where it will exact, through its fermenting, properties from its surroundings. Where the location has plenty minerals adaptable to building large frames from earthly matter, it reduces the mineral properties into a kind of plastic framework, or bone, and makes that a nucleus to build around, of the properties derived from suspension, yet not adaptable to the temperatures other 


\section{HEAT AND COLD.}

than by the chemical attraction generated by the fermenting process.

The fermenting process in its exactions and its detergent processes forms an organic body out of the plastic property. The exaction from inorganic properties where it has been deposited tending to build the framework of the new life, and in so doing making pores and arteries, veins and the like. The fermenting plastic properties becoming porous by the exactions from below earthly. And from above through deriving aid from the particles yet in suspension within the temperature; through chemical affinity within the plastic mass, and the properties yet in suspension. Thereby causing same to be precipitated, to again be taken up by the body in the building process. Such particles as come within the body not conforming to the work being thrown off by the excretory canals formed by the ferments. Just as we observe a fermenting mass today throwing off particles of matter, which make themselves very apparent to our olfactory nerves, if not to the windows of our brains. Thereby in the earlier process of fermenting the organs of exaction and excretion are developed by the fermenting of the mass. The pores and every other organ of circulation developing during this early stage of the life process. The same organs that are developed for excretion being made use of in case of emergency in the building of life by exacting from surroundings necessary properties which are within reach of attraction. The organs developed for exaction in the early 


\section{THE ADVENT OF MAN.}

process, capable of being made use of in excreting when necessary. The fermenting process being the product of a given temperature maintained for a given time. The property capable of being fermented being a compound of properties incapable of precipitation above a certain degree in temperature. Higher temperatures in the cooling process forcing the detention of like properties within the volatile form until temperature admitting of precipitation of the order of properties as a compound can be made possible.

In the case of the order of temperature having arrived on the high level plains that conform to the advent of man. The plains not having great bodies of mineral products adaptable to the building of great forms of life. Because the temperature had not arrived yet wherein the action of water could become manifest in aggregating bodies of salts and lime and like properties other than by the portions of same taken up by the actual compounding process of precipitation. These long distances of level highlands having an even distribution of mineral products brought down from the volatile world by the cooling process in the true order of relative precipitation. And in consequence, a form of life of medium between all forms of life physically.

The expanse of territory or level highlands being very great throughout the world. Cooling being very slow, owing to but one means of cooling, and that being radiation from the great expanse of plains in the one direction, outward toward space. In consequence, long 


\section{HEAT AND COLD.}

periods of constant temperatures. In fact, many years of cooling before the locations would admit of any other order of life of the organic order. In consequence, life adaptable to the order of nature as observed in like locations or circumstances.

Now, placing ourselves on the ground in the circumstances that brought forth the order of life we represent, it requires only a study of the man as revealed today to trace out the course of nature in the building of that form of life. Taking into consideration the build of man as revealed today. His habits and development. Means of sustenence during early life. And not being forgetful of the order of nature in its relative laws of precipitation, and the laws that made precipitation the order of the time.

The order of life being precipitated first within the circumstances made possible by the modified interaction between heat and cold, one contending with the other until the weight of the property, or properties in relativity became unadaptable to the domains where detained by the mean of conditions arrived at between the two forces. In consequence, the world of volatility was no longer the world adaptable to the property, and the property or properties, going toward forming life, was transformed into a world of material adaptable to the solidifying regions. Having arrived within the solidifying regions the properties being of an order that would ferment within themselves. Fermenting being an order of generating heat by chemical processes. These chemical processes taking up properties 


\section{THE ADVENT OF MAN.}

from surroundings necessary to the carrying out of the heat generating mechanism. Heat generating mechanism being adaptable to the laws of heat and cold. Heat when continuing, having to comply with the laws of heat and cold. Heat being a power when continuing that must have an outlet to throw off the reduced particles of matter. That is, particles of matter dispersed so as to become too impalpable to admit of further dispersal. In consequence, not being further adaptable to feed the heat producing process, and must be thrown off. In throwing off the reduced particles which were dispersed as far as the temperature of heat would admit, there had of necessity to be an outlet. The outlet in the mass must be forced by the lifting power or dispersing power of the heat generated from within. Forcing the refuse matter to break through the other properties of plastic matter forming the body of the organism. After having broken through once it kept the channels open by the chemical generation of additional heat seeking liberation, having been dispersed so far as to render the property useless in further separation. Rendered useless for further separation within the degree of temperature would render the property useless as food to generate heat, and it must be thrown off. These particles having forced an outlet in the mass of building material. Then the outlet was kept open as long as heat continued to be generated within the organism.

The feeding process was brought about by the chemical action of the process, varied and controlled 


\section{HEAT AND COLD.}

by the laws of heat and cold. To continue and thrive at a medium of adaptability to such processes. To wither and cease to continue, or die, when one force gained the ascendency over the other in relativity.

In the feeding of the early forming organism, its chemical properties exacting from surroundings all properties conducive to its welfare or to its growth. Exacting, through chemical affinity, properties on the earth's surface and molding same to form a nucleus, or bone, around which it could build up another form of body to be derived from the aerial volatile properties. The exaction by attraction of material properties from earth being upon properties necessary within the temperature in molding and stimulating the organism. Also tending to aid in feeding the organism. The properties derived from the volatile order being properties yet incapable of precipitation other than by the chemical attraction generated by the processes. Demanding same for food and transforming same into compounds of an order that has since been known as the animal flesh. The canals forced in the organism in feeding same from aerial properties being forced by the cooling action on certain properties acted upon by cold. Cold molding particles of material into certain volumes of size, or dimensions, necessitating a certain amount of heat to disperse. The same being precipitated from the aerial world. In the precipitation generated by the fall of bulk and further aided by chemical attraction when impinging against a mass of very plastic matter of a jelly-like order, they. 


\section{THE ADVENT OF MAN.}

were forced inward by sheer force of fall. Making pores or canals on their inroads within the mass. When sinking as far as attraction chemically considered would admit, they were subjected to the action of heat of the mass generated from within, and were further dispersed in conformity with the power of heat. Thereby compelled to disperse by the conditions inward, owing to least resistence, until they arrived at the final goal of utter dissolution within the power of heat generated within the organism. Then were thrown off through the refuse canals, as before mentioned. In all cases the feeding and dispersing of refuse going on until life or action generated within the organism ceased to be, owing to death of the order of life. All feeding processes and excreting processes tending to form all of the pores and blood arteries and veins, which we know today as means of sustaining the organism.

The human organism conforming to like conditions until today, and revealing every period of its history as does planets. Every organ, every pore or member of the human organism, tending to tell the story of its making, and not forgetful of the great story actions and means of life have to do with our past history. Our means of thought tell us a great story. Our period of development during early life tells a long and sad, but very true story as to our present superiority. In fact, the utter helplessness of our ingress made our exit in most all ages greater than other forms of life. 


\section{HEAT AND COLD.}

The period in worldly cooling and the portions conforming to man's place in relation to other forms of life, by the entry into existence, admit of no water on the face of the earth at that period in like parts of its surface adaptable to man's entry. In consequence, his development, to admit of his form of life within like conditions, and his slow development accordingly.

Man has a skin bare of any hair. It is of a chemical composition capable of carrying a temperature far below the temperature of surroundings aerial or otherwise. The conditions that brought man into being were in an early period of worldly history. The long plains were not cool enough to admit of the precipitation of water so as to detain same on surface. The earth was always secluded from direct action of the sun in like locations, owing to the great amount of matter and mists of the aerial order. In consequence always about the same in temperature. The sameness of temperature would render the precipitation of sufficient water on the face of the earth for detention an impossibility. The dark and heavy mists, enwrapping mists encircling like abodes, owing to temperature necessary in the conditions, rendered the water exactions as a maintenance of life a matter of exaction from the mists through the pores of the body. Instead of taking in gulps of water through the mouth.

Owing to the foregoing conditions which conclusively brought man into being, he developed as we know him, with a skin chemically capable of lowering the temperature below the surroundings. He having 


\section{THE ADVENT OF MAN.}

developed pores or in roads into his system in the early fermenting process. Consequently, his skin adaptable to the conditions that brought him into being cooled the mists or caused the precipitation upon same. The mists, having precipitated, carried nutriment chemically required within the organism. The pores took up the properties of water and nutriment and carried same into the organism.

The development of the brain during this process had to be wonderful, owing to having to pass judgment on all the properties of food and liquid entering the body. The conditions that brought man into being, as before mentioned, were the same that made him intellectually superior to other forms of life. More correctly, conditions developed him further than any other form of life.

Men having to depend on aerial properties for many years after life became a fact in his case, for food and nutriment for his whole system, in consequence he developed a very sensitive form of nerve system. The nerves being capable of exacting from anything they came in contact with and conveying same to the brain. The brain being a nerve center where the nerve conveyed particles which were extracted from particle of contact, the brain passed judgment on the case. The brain center having absolute control over the nerve system and also the muscular system, and in consequence used same as means of preserving the organism from foreign properties which were poisonous to the entire system. This being na- 


\section{HEAT AND COLD.}

ture's provision for the perpetuation of the species. And in consequence, the development of the brain and sensory nerves, aided by muscular department, all working to one end-perpetuation within the conditions.

In the premises the brain had to develop into an organic compound capable of distinguishing between the poisonous and the system-building properties, which had been precipitated upon the skin. The sensory nerves being made out of chemical properties that would exact from contact and carry same to the main office, or brain. The main office, or brain, passing judgment on the property and condemning or accepting same as the case may be. When rejected the sensory nerve conveyed a compound to the muscle, causing same to contract and throw out the chemical of a given kind. The compound sent to the muscle not affecting the entry of other properties of matter, providing that the dangerous element was not plentiful enough to necessitate the final closing of the pore or pores, for great periods of time. In that case, greater heat being occasioned within by the partial denial of passage to particles of reduced pabulum within the system and finally the transformation of the port of entry into a pore of excretion. When transformed into a means of excretion, the particles being carried outward, also carried the offending substance along in their wake. When the dangerous particle became removed in this manner, then the brain formed a compound from within and sent the same by sensory nerve 


\section{THE ADVENT OF MAN.}

to place on the muscle to offset the former compound and cause relaxation of same, allowing the entry of particles favorable to the organism, or food for reconstruction. And food for the general system. The reopening of the pores caused a reduction in the temperature of the skin and caused precipitation from the mists of particles that would admit of precipitation in the given temperature. Consequently did admit of the system taking up food for reconstruction.

Man had no other means of food for many years after life became a fact, only through the pores and other arteries. In consequence he developed very slowly. Developing slowly, he developed the brain more than any other part of his organism. The brain being in that age the principal means of carrying on life's process, guarding every entry and exit within the system. One moment, in cases, being sufficient to destroy the organism by the entry of poisonous properties held in suspension.

The stomach, mouth, eyes, ears and other organs of like order were some of nature's provisions for further development when conditions would become manifest in after years for the perpetuation of the order of life. During the earlier years of the life of our order that style of development was not in demand, and for that reason awaited in storage for time and necessity to prove their calling.

The stomach, as we observe today, can be transformed during the medium voyage of an individual across the stormy path of life, from a slim and nymph- 


\section{HEAT AND COLD.}

like being where muscular outlines are the predominant feature, to a being distorted by the abnormal development of the one organ. That one organ being the dominating feature of the alcoholic glutton. The stomach dominating the brain, by calling all its forces to bear by registering every impression from within. Oblivious of all earthly surroundings from the outside world. The organ that in the early forming life measured all space and matter in deriving nutriment for sustenence has been transformed by time and change in conditions to a seeking inward for feeling, admitting of more of one article of food to the extinction of all other forms of food. Consequently, loss of power on the part of the brain. The brain seeks but one article in nature now to maintain life and feed the glutton. In the forming process it knew no bounds; all nature was its field. It weighed every property in nature and judged of its properties of sustaining powers. It was unerring in its judgment. Every decree was irrevocable. Unrelenting was its verdict. But it was personal perpetuation that caused it to pass judgment upon all nature. It was not gluttony. Thereby showing that man has in fact, as a whole, in principle deteriorated. As he has unquestionably done by gluttony in his allotted span of life.

Eyes, ears, fingers and other organs, when observed across the span of life allotted to man as an individual under the conditions we are living under, will prove conclusively when observed throughout a life of a particular being, that they subserve the conditions 


\section{THE ADVENT OF MAN.}

subjected to. They being nature's safety valve in case of emergency. When emergency arises, then they come into play. If it never comes to pass that circumstances require their use, then they are no load to carry. They develop to the circumstances. Not the circumstances to the members. The same with man in the early life he developed to the circumstances, not circumstances conforming to his desires. Such members as nature allowed man in reserve which were not brought into play in early life were, as in all nature's work, provisions against future changes which are a part unavoidable in the fundamental laws of her working system. Nature having a law or laws moving to one end. Every form of its work conforms to the changes within the law or laws. Every manifestation of nature in molding processes as in all other processes, has been secured by not one means but many, for continuation throughout the changes which are bound to come to pass.

Man's thorough grilling in the beginning, having to develop so slowly and measure all worldly matter, was but the work of nature in adapting him to a future wherein he had to continue to measure matter and to do so unerringly in order to maintain matter in condition to the perpetuation of his kind. He came into the world in the beginning, a scientist empowered with all knowing qualities. He could measure all matter. He had to do so in order to perpetuate his own life. In perpetuating his own life he worked toward perpetuation of the earth. 


\section{HEAT AND COLD.}

Ignorance and jealousy of his own kind, backed with individual gluttony oblivious of all outward impressions, hankering to inward impressions or habits exacting one article of food, destroyed his natural inherent knowledge of all earthly matter. Further aided by his fall in worldly knowledge by inward impressions and jealousy, he resorted to a method of groping for revelation from some being who existed in fancy. That mode of reasoning again corrupted and tended to mislead him from the true path of knowledge. Knowledge being properly in the impressions derived by our sensory nerves and transmitted to the brain, by contact with material properties.

Being further aided by the nerves of vision transmitting to the brain the sense of appearance. Again aided by the sense of sound transmitted to our brains by the nerves of the ear registering the impression by contact against them. In fact, every impression derived by man worked in measure of matter by contact with same. The impression generated by contact of a property against a pore, becomes registered in the brain by the exaction of particles from the property by the nerve, and consequent conveyal of same to the brain. The brain compounds other properties with the particle and thereby measures it unerringly.

In the case of a sound coming to the ear, the par ticle generating the sound agitates particles of the same order which are conveyed in the atmospheric waves. These particles impinge against the auditory 


\section{THE ADVENT OF MAN.}

nerves and are immediately conveyed to the brain; the brain compounds same with other properties of brain matter and thereby determines the composition of the sound generating property. The judgment is unerring, providing other sounds are not impinging at the same time, thereby confounding the judgment by various derivative results. The reason why the judgment is not always unerring now days being on account of so many harsh sounds being always impinging against the sensory nerves, or auditory nerves, that in compounding the results of impact there are so many agitating mediums impinging at once. In consequence the brain, after weighing all by compounding same, has to resort to conjecture as to which of the results apply to the given case.

In the case of particles impinging against the nerves of vision, they are immediately transferred to the brain by the nerves. The brain compounds same and derives a result which is unerring. But where there are several different particles impinging at the same time and all taken to the brain to be compounded, then the brain has to resort to conjecture as to which of the results, which are unerring, applies to each given case. And therein remains the confusion. The results in each case being unerring, but adaptability to place of each tends to bring confusion into the results.

The brain can be corrupted by trying to weigh nothing, that is, looking for revelation from corrupt gods who have been reported as giving instructions to brains. The brain in trying to weigh the impressions 


\section{HEAT AND COLD.}

which never come from nothingness, feeds on itself; and in consequence lapses into practically nothing. The brain's office is to weigh matter; weighing matter, it feeds on matter and thrives. Weighing nothing, as in the case of waiting for revelation, it feeds on itself.

Man when he leaves the field of matter and gropes off in the dead past, looking for a word from some one instead of taking impressions from what is really manifest, corrupts his brain. The brain being capable of a certain amount of work within a given time, like any other property of matter. If the time is taken up in groping into space and concentrating all its impressions upon that one subject, trying to glean a word from nothing and nothingness, it cannot work on the weighing of matter. In groping for the imaginary being who is going to tell the brain what is next, it loses its right to decide. It surrenders its right which nature has given it in measuring matter by impressions. It dare not pass judgment on the impressions transferred by the sensory nerves by contact, fearing that the great one will punish it for its temerity. In fact, it puts the ban on mankind as to perpetuation, because it denies him the right to weigh matter and work toward its molding into condition tending to conserve his kind. The great one as deemed by our gospel truths, as they dare to call them, means the extinction of mankind. Extinction of mankind means transformation of our world by withering and loss of heat. And a trip through space to aid in building up another body in space at some distant region. 


\section{THE ADVENT OF MAN.}

Man can continue the world or earth indefinitely by the powers nature has given him if he will exert himself in developing the qualities of brain in measuring and transforming matter so as to conserve heat and temperature adaptable to his kind. His brain has the office of passing judgment on all matter, and when liberated from groping into the shadows of past mythological conclusions, he will be supreme. He has it in his power to discontinue the process of life of his kind as a means of destroying the earth. He has been working along that line for thousands of years. But fortunately in the last two centuries there have been a few master minds who have taken up the natural process of weighing matter. Working along the lines of nature toward perpetuation in the face of powerful opposition.

The axiom of the fall of man proves to be very true when you come to read the history of man through studying his make-up; that is, when inquiring of nature as to how made and how changed, within the change of her form. Thereby seeking the period of history in the cooling process admitting of such changes coming into being. The laws being unchangeable, then it only requires a survey of change of form in matter complying with the unchangeable action which we observe to get results. And weighing all in the light of true nature's law, while surveying the corrupt and tyrannical code accepted by man as fundamental; and holding it in the background as an encyclopedia containing the true account and seed of man's downfall. 


\section{HEAT AND COLD.}

Man truly fell from his high state as judge of everything earthly; judging everything through the contact of sensory nerves with all matter, and they exacting particles from contact, with particle impinging; then carrying same to the brain to be compounded with other particles within, so as to determine the composition of particles coming in contact. The date can be conclusively placed at the day or time the tyrant took the throne and commanded man to follow his instructions. Man lost power of action within the brain from that day onward. The brain not acting upon the particles of matter and judging of same, tended to wither and remain within itself. It, when compounding particles of matter, was forced to do so within itself, fearing punishment for its temerity at the hands of the thief and tyrant who took the throne of command.

For years man was undoubtedly forced to obey the tyrant, but as the years passed the young were brought up under like belief and finally inherited the weakness. They looking for revelation from without instead of revelation from within the man by contact with the particles of matter and the laws governing movement of matter. They looking off into the mists of ignorance for the illegible characters written by the unseen hand of the royal arch tyrant, the coward, who secluded self as the means of precedent of everything bad in man, and threw out bait to man's weakness to lead him to his own destruction by looking for means of life in others instead of himself. Thereby making 


\section{THE ADVENT OF MAN.}

man aggressive, mean, contemptible and jealous of his fellowman.

During the early forming Process, man's blood was forced to circulate by the action of heat and cold upon the particles of matter which formed the man. Heat and cold in any organic or planetary body, generating heat within itself, have lines of demarkation between the forces. Heat contending from within the organism or planet for a line of working action with the cold from without. The medium established between these two forces being necessary in order to continue action of any order. In consequence, we find that the medium of demarkation or center of gravity in the continuation of action in the man or any other organic body becomes centered at the heart.

The range of action on the organic body where cold acts being on the outer surface. Cold congealing particles into given sized units. The units necessitating a given temperature to reduce same into more minute units. In consequence, the units formed by the action of cold sink through gravity to the line of demarkation or heat. Heat being generated from within the organic body in the lungs and other portions of the body acting chemically upon particles of pabulum; in consequence, separation of particles into the minor sized unit, owing to the highest temperature capable of being generated within the organism. Result: heat drives the minor units to the center arrived at between the forces of heat and cold, or line of demarkation. In consequence, the two forces contend 


\section{HEAT AND COLD.}

for equal rights to act in the premises or line of demarkation. The result proves to be action, movement. The two forces double on the movement at the line of demarkation and in consequence act in harmony instead of opposition. Thereby the pump for transporting the blood from the heart. While cold molds greater units near the surface which will sink to the center of gravity between the forces, by the force of weight of particles. molded by cold. Bodily heat in the margin being insufficient to reduce the particles into more minute units. They having to impinge against the mechanism of the mode of movement generated between cold and heat. Then become transported by pump movement to greater heat and reduced. 


\section{CHAPTER XVI}

\section{CIRCULATION OF THE BLOOD.}

The circulation of the blood through the human system, and of course all other organic systems, has raised a question in physics which confounds the greatest thinkers in the modern world of thought. The author being a disciple of the mode of thought adaptable to conditions manifest within the working order of nature, and not being an adept in the laws of accepted physics or in human anatomy. Yet he dares to show, and conclusively, that the circulation of the blood obeys the fundamental law of nature; namely, action of heat and cold upon the particles of matter.

Worlds in their movements obey the law of heat and cold, by being formed in the line of demarkation between the action of heat and cold. Cold being equal to heat by its action on the particles within the given locality and vice versa. Consequently the two forces act upon the particles of matter that form in the margin. One tending to disperse the particles of matter. The other tending to unify the particles of matter. The portion being subject to one force being diagonally opposite to the other force destroys the equilibrium and causes movement in the planet. The movement in planets being to turn about or revolve. The movement is continued by the continued action of heat and 


\section{HEAT AND COLD.}

cold upon the particles of matter forming the body or unit in question.

The action is similar in the organic body, only difference being a change in the movement when generated in the medium between the two forces. Wherever the two forces are equal, let it be in an organic body or a planetary body, the place of equality becomes a center of gravity. The heat generating power lifting by its power to disperse becomes equal in the margin to the settling or uniting power of cold when exercised upon the particles of matter.

In consequence we find that the heart is the point of medium forces in an organic body. Cold and heat contending for equal rights in the locality, and in consequence, movement. Each force doubling when the lift comes on the particles of matter; or in other words, acting in union to create movement. The movement being equally the work of cold upon the particles and heat upon particles of matter.

Now we will take up the question of circulation at the point of greater heat in the human organism, and trace its course throughout the human body until it makes its triumphant entry to the place of beginning. We will show cause for every movement throughout the course and why the cause.

In order that the reader may more thoroughly understand the beginning movement, we will explain first where the cold and heat act in union to create the movement. Then, as we carry the reader throughout the course of the blood flow, we will show 


\section{CIRCULATION OF THE BLOOD.}

how cold acts and becomes active at the heart in carrying on its part of the movement generated.

Heat and cold act conjointly in causing movement in the heart. The right auricle and the right ventricle are agencies actuated by the action of cold upon the particles of blood. They derive their power, that is, working power, from cold on the particles of matter. The left auricle and left ventricle derive their working power from the action of heat, acting upon the particles of blood or matter. Cold and heat acting in union in forcing each movement. Cold aiding heat in causing the forcing of blood through the aorta. Heat aiding cold in forcing blood into the pulmonary canals carrying same to the point of heat, or lungs.

Now to consider blood leaving the lungs: Blood leaving the lungs does so through being reduced by having all foreign matter burned by the greater heat generated within the human organism. This burning causes the dispersal of blood particles into the most minute particles possible within the heat generated within the human body. Dispersal by using waste matter for fuel in the burning process within the lungs, causes the waste matter to be thrown off by the lungs, on account of being of no more value as fuel. The blood having been cleansed of all particles capable of use as fuel within the given temperature, and in consequence being no longer of use within the lungs. It becomes subject to the dispersing power, or lifting power generated. Heat being a lifting power. Con- 


\section{HEAT AND COLD.}

sequently it is thrown off along the line of least resïstence.

The line of least resistence taking into consideration the degree of reduction in dimension of the particles, being toward the left auricle of the heart. The blood not being reducable by the heat generated in the mechanism sufficiently to pass through the pores of escape within the lungs, for waste materials. The lifting power of heat upon the particles of blood, aided by the accumulated weight of blood within the left auricle cause the biscuped valves to open, and in consequence the flow of blood continues to fill up the left ventricle. The left ventricle filling by the weight of blood flowing in causing contraction in the right ventricle and aiding the action of cold in forcing the blood collected in right ventricle off into the lungs. The blood thrown off into the lungs by the contraction of right ventricle becomes replaced by the opening of the tricusped valves, and they in turn cause the blood flowing into right ventricle to press in or contract the left ventricle, thereby causing the blood to move from left ventricle into the aorta. Having entered the aorta the blood flows along a course where heat continues to be generated sufficient to disperse the blood through the pores toward the surface of the body. This dispersing being but the law of heat in its lifting or dispersing power on the properties of matter. The arterial system being situated in the central portion of the trunk of body and being fed from every heat generating system throughout its course. Its natural ten- 


\section{CIRCULATION OF THE BLOOD.}

dency will be to disperse by the lifting power toward surface of the body. This lifting of minute particle of blood continues through minute passages that were formed in the fermenting process as a means of communication between the action of heat and cold in continuing life or beginning life. When the particles reach the surface they are acted upon by the cold. The cold molding the particles of blood into greater bodies, or units.

When the cold has molded the particles of blood when near the surface into particles of greater size, they no longer can be buoyed up by the action of the given heat in the locality or portion of bodily surface. They cannot fall or sink down into the minute passages where they were buoyed upward, owing to solidifying into greater units. Consequently they become subject to a new law, governed by cold. Cold having by its cohesive tendencies upon the particles of matter congregated the particles into greater units. The units being subject to a given temperature of dispersal. The units of a given size having a course in which they can sink by attraction generated by cold contending for limits with heat to a given point. In consequence the particles are driven inward by cold and attracted inward by the center of gravity or line where heat and cold are equal. In this course their action is uniform, owing to the weight of particles being equal after the action at surface. There being no means of separation of the particles into more minute units until a certain temperature had arrived, and consequently 


\section{HEAT AND COLD.}

uniformity in movement. The particles first settling through capillaries adaptable to the size of units forced by action of cold by its cohesive tendency. When entering veins still flowing with uniformity toward the point or medium of cohesive tendency or right auricle. When entering right auricle weight of accumulated particles forcing open the tricusped valves and entering the right ventricle of the heart. When entering the right ventricle finding the left ventricle filled with blood, forcing same inward by weight of blood in right ventricle. And in consequence, forcing blood which filled left ventricle to go into the aorta, or place of beginning of our course through the human body.

Again, when the lift is taken from the lungs on the heated blood or dispersed particles of same, they force the blood into the left auricle, its weight opening valves and causing the filled right ventricle to contract by throwing blood which flowed in through the veinular system into the lungs.

As a matter of course, the action of the cooled or heavy particles of blood flowing inward from the surface of the system, by the action of cold, act simultaneously with the lift from the lungs on the blood which has been purged of excessive weights of material. The inflow of the blood from surface aiding the forcing of blood into aorta.

The blood, when forced into the lungs from right ventricle, is not only lifted by the accumulated weight in the right auricle, but. is aided by the weight of all the blood in the left auricle bearing down on the blood 


\section{CIRCULATION OF THE BLOOD.}

in the left ventricle; all tending to aid in contracting the right auricle for the lift. The blood forced into the lungs by the lift from right ventricle aids the lifting power of heat generated by dispersing all blood from the lungs into the left auricle. And thereby the inflow into the lungs becomes an additional force in lifting blood toward the left auricle. So in every lift, let it be into the lungs or out of the lungs, the cold and heat double in the lift. Or into the aorta as the case may be. In fact every action or movement is generated by a doubling of the action of heat and cold, that is, when considered in nature's work. Where she herself molded the action, or the movement. The movement is always generated where the action of cold upon the particles can be aided by the action of heat on the particles of matter. Wherever the two can congregate or concentrate their powers upon matter, movement and life must be the result.

We find by inspecting the researches of our kind in the order of composition of blood within the veins and the arteries, that they conform to the necessary dimensions, arterial and veinular. The blood in the veins being greater, considered as units, both in weight and dimensions. When considered in the arterial system it is more reduced in size of unit flows with greater rapidity in the arteries. It loses particles which will be of service in creating heat while in the lungs. It is colder near the surface and continues to be so until put through the burning process within the lungs. The particles which have been burned or separated so as 


\section{HEAT AND COLD.}

to be rendered worthless for further food while in the lungs, are thrown off through the canals of excretion, and are properties dispersable in the temperature generated within the lungs. All tending to prove that man had discovered the true law years ago, but was incapable of putting two and two together and acquiring the true result.

We will weigh the blood of humanity in the conception of beings as a further proof of the law of heat and cold being the supreme law of nature. We will show that even in the conception of life as we observe it that the law of heat and cold must be consistently and conclusively the arbiter of our destinies. We will not go off into imaginary ravings, but take our groundings on the truths derived by research by our fellowmen as a foundation. We will name no authorities but leave that to the searcher for the truth to seek.

The blood of man being much more dense than that of woman; being more dense means colder. The law of nature never varying on a property in nature of a kind in condensing same. Every property precipitating at a given temperature. Consequently, if man's blood is more dense, it must be colder than the blood of woman. Woman's blood being more rare or attenuated than man's, she must be capable of generating more heat in order to maintain the attenuated condition of the same particles of matter. Taking that as our base of action, we will find the action of man and woman in union becomes the union of heat and cold. What is the result? One being colder than 


\section{CIRCULATION OF THE BLOOD.}

the other, must bring about a center of equal powers between the forces. At the junction of two forces of heat and cold, or point where they neutralize lines of aggression, or become equal upon the particles of matter, life and movement should be the result. It has been the result of every study in which we have considered its action in coming together on the particles of matter. In the case of blood becoming more dense near the surface of the being it flows toward the heat to be neutralized and in doing so comes to a line where heat has been neutralized. But there has been formed, in consequence, in every case, a means of movement capable of doubling the powers. In the case of man being colder than the woman, the blood should flow from the colder to the warmer body until a line of equality should be reached. When the line of equality between the two has been reached, life should be the result. Movement of an order and life. As a matter of fact that is the case; the blood flows from the colder body to the warmer, and when work is equal, life is the result.

In the case of the woman carrying the foetus, her blood becomes more attenuated. But the young life growing has still more attenuated blood than the mother. Tending to show that the mother has generated more heat during this period. But also tending to show that the youthful being has become a source of additional heat in itself. Its heat being greater than that of the mother. In each case conforming with the working laws of nature. 


\section{HEAT AND COLD.}

The fact of the child expanding or growing would necessitate additional heat within the laws at the foundation of heat and cold. As heat tends to expand and cold to contraction. on the particles of matter. The mother also expanding in the true order of nature in order to make room for the growing being, should, according to the laws of heat and cold, raise in temperature. The reduction in the density of the blood tells the true story of temperature in both cases. It is a question as to whether our means, mechanically considered, would weigh as thoroughly the condition of temperature as the condition of the blood.

The blood being incapable of lying in the case. As the same properties of matter when subjected to temperature, the temperature being the same, will always act the same. 


\section{CHAPTER XVII}

SECOND PERIOD OF INTELLECTUAL DEVELOPMENT IN MAN. - DEVELOPMENT OF THE STOMACH.-FEED FOR THE WOLVES.-CLOTHING.

Man having developed first to live by the exaction from contact with properties in nature yet in suspension. As nature cooled the properties tending to keep up life being precipitated in their order of precipitation. In consequence, the properties going toward feeding man had to be derived from earthly properties formed out of the same properties he formerly derived from aerial contact. In consequence, he began to use his stomach in digesting the chemical properties made up from the former articles of diet. $\mathrm{He}$, being a judge by contact, could distinguish between the dangerous and the necessary articles of food.

But at the period of the stomach coming into play another dangerous enemy, more dangerous than the poisons derived from aerial contact, came into his line of contact. These being the carnivorous droves from the mountain districts. The cooling of nature upon the properties of matter made water in man's abode a matter of course. Water being detained on the surface rendered the life of the mountain fastnesses very neighborly by their visiting man with intent to feed on him. 


\section{HEAT AND COLD.}

Man being helpless physically when confronted with the powerful animal life of the mountain districts. And for that reason made very good food for the carnivorous form of life. In fact he became the principal diet of that form of animal life. He was at first incapable of protecting himself in the face of such power. But early training in judgment of what was dangerous and what was adaptable to maintain life led the way to his final supremacy. He having developed the brain in carrying on his mode of life. And in consequence he resorted to strategy in overcoming the physical force.

He had nature for his guide as in the former battle. He had personal perpetuation as the stakes. He noticed when he attempted to run that the powerful carnivorous animals were superior. In consequence, it was useless to resort to flight. He noticed that when he ran and stumped his toe against an impediment he would fall. There was an outlet for him; he would trip the animal, as nature instructed him by impeding his progress. He followed nature's instructions by complying with the lesson derived from nature. He tripped the animals; he made meat and clothing out of the dangerous foe. What was once his deadly enemy became his food and clothing. In fact, became a necessity in his own preservation.

The battle was won by the brain over the physical world at this early period in human history. It transformed the greatest scourge into the greatest benefit to mankind. In fact, it was the saving of mankind. 


\section{INTELLECTUAL DEVELOPMENT.}

He being physically unable to coup with the rigors of climate much longer without means of covering. Because nature had cooled so as to be inconsistent with his means of life without his adaptation to the conditions. The enemy gave him the means of both food and dress.

The enemy did not alone give him food and dress, but also developed his brain likewise.' Opening new avenues of thought. The enemy was the means of bringing the destruction upon itself by suggesting to the man, through necessity, the means of its downfall. The stakes being so high that it suggested to the man every means of thought in overcoming same. If the carnivorous animals would not have bothered man or attacked him in pursuit of his life, he would never have attempted to destroy same or use the flesh for food and the skins for dress. And man would have perished. But the stakes made the avenue a necessity. Man acted; he won. Man is not to be credited with the overcoming of the great foe, but instead the conditions that brought him into being; and further, the conditions that confronted him in maintaining himself. He was the product of conditions and had to grow to the conditions. Further conditions having forced him, he had to contend with them. It being nature's law to try and perpetuate kind. It being nature's first law to preserve life in the face of every and all dangers. Man was born under adverse conditions that he may develop his brain to perpetuate himself and kind. In doing so, nature always suggested the means 


\section{HEAT AND COLD.}

of winning out. He followed the suggestion; he won out. Nature suggested to him by leaving him hungry. Nature suggested to him by leaving him physically incapable of contending with greater force. Nature suggested to him by leaving him in danger of being made food of by great animals. Nature suggested to him by allowing him to see by the eyes given to him by nature, the eating of his kind by carnivorous animals. Suggesting to him, you are next. Nature also suggested the means of his tripping the powerful animal. Nature also suggested the means of his trapping same. He looked, he thought, he acted the part of the suggestion, and he won the battle.

A great danger, when manifest, a great wrong, when perpetrated, in any age of the world's history, works the destruction of itself, by the means it suggests in the brains of the victim, whom it forces to act along the lines suggested by nature in the final crushing. When the brain, or particles of matter that rounded out the foregoing conclusions in this paragraph, has been liberated within the elements, and the elements a million times transformed. Still the truth of the conclusion shall remain untarnished, while backed by the experience of ages.

This period in the development of man, when he was confronted with the great droves of carnivorous animals, forced man to commune with man in the general battle of preservation of mankind. He had to act in conjunction with his fellowman in order to preserve self. It was nature's method of uniting man- 


\section{INTELLECTUAL DEVELOPMENT.}

kind so as to act together as a unit in the main cause which man was born to fulfil, namely, worldly conservation. He deriving the first lesson in union by the application of nature's first law, namely, personal preservation. And from that day until this it has always proven to be the first law of nature; not barring its application to man when he becomes the danger to the existence of another. 


\section{CHAPTER XVIII}

\section{THE OFFICE OF THE BRAIN.}

The brain, mechanically considered, is a balance, or scales, capable of weighing everything in nature. It is nature's greatest work; in fact, it is the only work that has arrived at perfection. It is the product of a condition. And the product of the most adverse conditions proves to be the masterpiece.

The brain knows no superior; it is omnipotent in its office. It degrades itself and its powers by taking a command. A command is of no value when subjected to the action of the brain in weighing it, unless the command comes from the source that made the brain. In that case the brain does not take the command, but instead it passes judgment by weighing the command. If the command weighs out, it is accepted. If it does not weigh according to the laws that formed the brain, then the brain condemns it. And the condemnation is just. The brain can always find the tyrant by exercising the powers of its office in weighing the data that comes before it. Let the data be in the form of command or suggestion, other than by the laws of nature that made the brain a possibility. The laws of nature are always manifest. They are not secluded behind the ramparts of superstition. They need no interpreter other than the omnipotent mechanism de- 


\section{THE OFFICE OF THE BRAIN.}

rived from nature for balancing the impressions existing.

We have a father and mother in the identical form we represent; we know that our fathers and mothers are our progenitors. We know that our fathers and mothers are our best friends. We know it from the long years of self-abnegation on their parts, in administering to our personal welfare and wants. We would not for one moment listen to a voice from some traveling stranger telling of the command of some unseen friend and creator who instructs through the stranger, our utter disregard of the commands or instruction of our dear fathers and mothers. We would immédiately condemn the traveling stranger as an imposter who dared to preach the commands of the unseen and unfeelable. We would immediately condemn the hidden stranger who slouched behind the ramparts of seclusion and gave his commands. We would term him, if he lived behind the ramparts, in fact, a coward, a villain, a traitor, a tyrant, who dared not face the light of judgment in the face of the measuring mechanism we are empowered to carry. We would weigh his method of command as identical with the method resorted to by the vilest assassin, who awaits in seclusion behind some rock or other rampart the coming of some passing mortal who knows not his hiding, and who becomes victim to the deadly being in waiting. We would weigh him in that light and just would be the judgment in the face of the method he resorted to.

We can exercise our weighing powers of our 


\section{HEAT AND COLD.}

mechanism in judging any and all events that have preceded us in worldly action, by the marks left, by the work done. No work being possible, in nature, of transpiring without leaving a record on some particle of matter, that will be within the domains balanceable by the mechanism of nature's balance. Nature's balance being such a thorough mechanical contrivance as to detect any and all differences in weights, and weigh so thoroughly as to find where the missing link would be applicable by its nature.

Our mechanism being so perfect as to descry our creator from the simplest of our observations. In fact the only danger that confronts us in seeing our creator being our method of looking off in the distance brought about by the surrender of the powers that are ours by right. Observing the simplest of nature's products and laws leads the balance to weigh all past actions in the scale of passing action. And all nature conforms, thereby rendering our creator the most omnipresent of all existing things. Therefore we cannot touch or think or move without being in contact with our creator. Our creator being heat and cold upon the particles of matter. Call it God, devil or change as you like, it is still our creator. We cannot see anything or feel anything, hear anything, do anything, smell anything or taste anything but what is part of the creator. Because cold and heat with matter covers all the senses.

We can bring the balance to bear on the passing action and weigh it; then we can transport passing 


\section{THE OFFICE OF THE BRAIN.}

action down the vista of buried ages; down the vista of buried worlds, yes, millions. When transported, passing action, as we observe it, becomes placed as we know it today, acting the same way on the same properties of matter; in any world or during any period of its history we can weigh the condition as if today. Our power of weighing the action of the omnipotent has no limit, either behind or before us. We know what it has done in the past by the way it acts before our eyes. We know what it will do in the future by the way it acts before us to-day.

We can study passing action as manifest to our senses today and carry the action down the buried ages until we see, not in fancy but in fact, our molding in the passing action. We can see every passing change until the organism is completed. When completed, we can follow passing action along its course, weighing everything that comes to pass throughout all the ages, until we arrive at the period where we are manifest in fact as a living personality. Or, to the poirt, where we live in worldly history as a being, breathing, feeling and seeing the passing action.

The omnipotent's greatest enemy, the greatest rebel, the greatest coward, is the man who sits upon a throne of personal manufacture, and dictates laws, commands to fellowmen, asking them to utterly disregard the dictates of nature as manifest. Commanding men to rely on the truth of the command without subjecting it to measure in the scale given to man by the creator. $\mathrm{He}$ is the devil as we are led to believe, by modern 


\section{HEAT AND COLD.}

thought to be the enemy of society. If devil is the name we shall know the enemy to society by, then we must transport the name to its rightful owner. The party who dares to deny man the right to judge in himself of the evidence presented is the devil. And man should use his measuring mechanism in finding him by weighing what he sees, feels, smells, hears and tastes. And weighing all in the scale of passing action, not in the scale of revealed myths of past ages which are means of putting the tyrant on the throne. Or more properly the means of man's downfall. Man knows no superior, only the means that made him, and man cannot find in his senses other than his maker. Heat and cold upon the particles of matter, and you have your maker, your God, your omnipotent, your deity or any other name you desire to call the power.

Should current thought be unwilling to accept the truth of the assertion of the maker_being the abovenamed power, then by all means weigh the powers by the mechanism of the brain, not in the scale of revelation bibular. If found wanting after thorough weighing, then by all means disregard the truth of same. If when weighed in the balance they prove to be all there is of maker and capable of every action. Then man, know thy maker.

When knowing and seeing, then discontinue the teachings of the enemy to society. But by all means do not debar the enemy until proof is conclusive of it being the enemy to society. Because no amount of recompense or retribution can ever right a wrong. 


\section{THE OFFICE OF THE BRAIN.}

The brain is a compound formed during the fermenting process. It is of a composition that takes on or throws off of the material which comes in contact with it. And in that property which it displays is the real secret of its perfection in weighing matter. When the olfactory nerves come into contact with a substance, they convey particles of the material to the brain. If the particles are injurious to the system in the judgment of the brain, which is always correct, then the brain compounds the particles with particles of brain and transports them out of the system by the nerves. The nerves conveying the refuse made of brain and the particles to the muscles around the olfactory pores, the muscles throwing the particles out and closing the pores. When the particles in like case are agreeable when conveyed to the brain by the contact of olfactory nerves, then the brain assimilates the particles. Also sends down a message by contact of olfactory nerves to open pores and allow the necessary food to brain and body inward. In creating this action, the brain sends down particles of brain matter that relaxes the muscles of olfactory passages.

In the case of particles being impinged against the auditory nerves, caused by the impact of particular substances. The substances impinging against each other causing noise. The particles of each given make tends to rustle or impart movement to particular particles detained within the aerial waves.

In the case of the particles being iron, which impinge against each other, they impart movement to a 


\section{HEAT AND COLD.}

particular property in suspension which conveys sound. This property impinges against the auditory nerves. The auditory nerves carry particles which impinge to the brain. The brain assimilates the particles maintain same. When injurious, the brain transforms the particles by compounding same with particles of brain matter, and transport the compound to the muscles. The muscles convey the injurious particles outward.

When a particle of matter or a condition of temperature becomes registered by contact against the sensory nerves, the sensory nerves transport particles to the brain. If not injurious, the brain allows same to enter brain and body. But when judgment is passed by the brain terming the particles injurious, then the brain compounds the particle with other properties within the brain and sends it out on the mandatory nerves, and they turn it over to the muscles, which force it out of the system and close the gates to further entry.

When particles of matter impinge against the nerves of vision, they are immediately transferred to the brain, and when termed non-injurious to the system are allowed to impinge with impunity. When termed injurious, the nerves convey a compound generated within the brain to be imparted to the muscles, which draw a curtain over the passage to deter the entry of great amounts of the dangerous element.

When particles of matter come into contact with the nerves of taste, the nerves immediately become loaded with particles detached from the property and convey same to the brain; when non-injurious to taste, 


\section{THE OFFICE OF THE BRAIN.}

the brain leaves all doors open. When found injurious the brain forms a compound out of the particles of matter and brain properties, and has same conveyed back to be thrown on the muscles to cause same to contract.

In all cases the brain is compensated for the loss within itself in the compounding process. It always assimilating particles that are beneficial which are conveyed to it.

Notice when you have sat down where the scenery is magnificent and all nature seems to love life in its varied forms. Did you ever notice the all-pervading self-satisfaction in being alive under some such experiences? Do you know why? It is because where nature has life of varied forms, all collaborating to the one end, there is more food for the brain. The brain rejecting the undesirable and throwing open all avenues of entry to the desirable. The brain takes on food and reconstructs its wasted condition during these complacent hours. Just as the stomach brings about a satisfaction in being alive when health is perfect and a square meal has just been shared. It is the same with the brain; it gets its greatest amount of food along the nerve senses, and where life is varied and beautiful; the brain will rest with satisfaction by being crowded with every desire in its reconstruction.

In all the five senses the brain closing the gates of entry, passing judgment on particular chemical elements, and loading the muscles with the means of a compound capable of contraction within the range of contact with the injurious substance. When not in con- 


\section{HEAT AND COLD.}

tact with the injurious substance they are open. The compound being actuated by contact with the offensive chemical property, and when in contact tightens the grip upon the opening. The brain judges all matter and never errs. It compounds a preventive of entry in all cases and conveys same by the lines of transmission of particles inward or outward.

When man will get back to his proper place in judgment of eatables as well as religious belief by listening to the call of the brain, then life will be long and prosperous. Happiness will be his, with the long life which must be the consequence. Death to superstition. Long life to the judge who was throned by our maker. The brain is king for once and forever.

- Lest we be assailed by those who place life and movement in the hands of the miraculous, as is their wont, we shall further describe the action of the brain in exaction.

Lest they ask how it is that the brain can exact from the properties of matter which will not volatilize in the given temperature of contact with sensory nerves? Will answer: it is not necessary for the brain to exact but one element from all matter so as to determine the consistency of the property being questioned, because there is one property in nature which goes toward making up every compound in nature, and that property is present in varying quantities in the brain.

Of course the brain exacts more than' one element from matter, but it would not be necessary to do so, so 


\section{THE OFFICE OF THE BRAIN.}

as to weigh all matter, because heat and cold, acting upon one element, would render the brain omnipotent in its office in judging all matter.

The amount of that property exacted from contact, or thrown off by contact when influenced by the action of heat and cold upon the particle or element, will always give the true result as to class of property so affecting by contact.

In the case of the compound coming into contact with the sensory nerves and being colder than an element in the brain, then particles of the element in the compound will move toward the element of like kind, tending to equilibrate temperature. The amount moving toward the brain when weighed will give the true result as to kind of compound so effecting.

When the compound coming into contact with the sensory nerve is of a higher temperature than the brain, then the element will move from brain toward like element in particle effecting, tending to seek level or equilibrium of property of a kind. In doing so the amount given off by the brain of like element will give the true result or weight of property being questioned. Thereby heat and cold acting upon the properties of matter in the case of the brain will weigh all matter, as well as make the man out of the gases. In other words, the maker always gives results when questioned. The maker being heat and cold acting upon the particles of matter.

Abstract conclusions are arrived at by the brain balancing results derived from different contacts with 


\section{HEAT AND COLD.}

properties of matter, organic or otherwise. Results or weights being a form of law, balancing of laws give abstract deductions. Or a law derived by weighing law.

\section{CHAPTER XIX}

\section{TEMPERATURE VS. INTELLECT.}

Heat and cold acting upon the particles of matter make the man, and also mold his intellect in proportion to the mean of established action between the forces acting upon the developing organism.

Man therefore becomes great intellectually as he becomes adapted to communication with the maker; or more properly, subjected to growth within the interchanging action of the creating forces.

We can resort to history as our reference in proving that what is theoretically necessary also proves to be actually correct, when studied from the pages left of man's work. History shows that in the extreme north where cold is predominant, that the man throughout all ages has been sluggish in mind, he being very slow to act. He being very slow to concentrate mind to a point carrying out an action tends 
to prove; the dominating power controlling his action. Cold tending to concentration or cohesion.

In the extreme south, or where heat is predominant, taking the year around, the mind is flighty, hasty, and will not for one moment hinge when action touches the senses. The movement in mind is spontaneous as the action of heat in separating particles by combustion. All tending to prove the true law of heat and cold being the dominating features of action of matter.

In the middle regions between heat and cold, where the two become equally effective upon the forming organism, we find our greatest intellects. In this case tending to prove that man reaches his greatest perfection when subjected to the interaction of all three forces of his maker. That is, the matter forming his organic body being subjected with equal force to the action of heat and cold.

Wherever there has been a great intellect in a far off southern region where heat predominates, it will be found from the pages of history that the mind was the product of a mountainous region during the greater period of the building process, going on within the brain. It will be found that wherever there has been a great intellect in a far-off northern region, where cold was the predominating power, that the intellect was the product during the forming process within same, of low lands. Thereby tending to prove that the anomaly in either north or south, when subjected to inquiry, will show the same result as the mid- 


\section{HEAT AND COLD.}

dle regions. That is, cold and heat being made equal with regard to the forming organism; or in other words, nearer relationship to the creator.

The mountainous regions in a southern latitude produce the same climate as the middle regions of great intellects. The extreme lowlands in northern regions produce nearly the same climate as the middle regions where we get our greater intellects from. So we see it is not an anomaly at all when we get a great intellect in the extreme north or south; but instead is nothing more than the obedience to the true law of our maker. Being in communion with our maker every day of our lives, and being equally subject to all three forces will without question bring about better results than when slighted by one of the powers of our making.

The same laws will apply to the physical construction of mankind, where the forces are equal or nearly so, we get our greater physical structures in the form of men. Not greater in bulk, as that would be against the law of heat and cold, but greater in endurance and strength, taking into consideration the bulk being equal. The greater bodies also conform to the laws of heat and cold. In hot climates the greater bulk of body. In extremely cold climates in the north the smaller forms on earth. Heat tending to expand, in consequence greater forms. Cold tending to union of all matter and in consequence cohesive tendency becomes manifest in the living organic body as well as all earthly products. So in final we may subject cold and heat to trial within all earthly action and all life's 


\section{TEMPERATURE VS. INTELLECT.}

products and get the same result. Expansion for heat. Contraction for cold. And great endurance and great mind where the two are equally manifest on the properties of matter.

Particular races which prove to be small in a hot climate, like the Bushmen of South Africa, can be accounted for by the scarcity of the mineral products going toward forming the nucleus, or bone, during the process of forming life, when the earth was cooling. And the temperature conducive to life was present. 


\section{CHAPTER XX}

\section{MORALITY.}

Morality, when weighed in the light of nature's law, proves to be, "Do unto others as you wish to be done by." Wherever that quotation was derived from properly, there was a true knowledge of nature. The author of that passage was a true student of nature. He was not a God. He was a man who exercised the proper power which nature had given him. He was simply acting according to the dictates of the power that made him. He exercised the proper power of the brain; he weighed passing events or action in the balance and from same derived truth. His truth, made ponderable in those few words, will live throughout the ages.

Now we will weigh that passage in the light of passing action and find what the result will be at this far off period in worldly action, from the origin of the saying. Cold and heat molding matter into different forms, each endowed with the desire of personal perpetuation. The perpetuation of all being weighed in the law of passing action, is found to be accessory to worldly perpetuation. Each acting with the means it has been given by nature in transforming latent heat into active heat tending to one end. That end worldly perpetuation. 


\section{MORALITY.}

Man weighed in the scale of passing action proves to be the greatest of conserving powers in nature tending to worldly perpetuation, because he liberates more heat toward conserving the world than all the other forms of life. In doing so he uses other forms of life as accessories in carrying on the good work. He being greater through the process of nature that brought him into being. Nature bringing him into being for the purpose of worldly perpetuation. Nature subjected him to the trials in early life, that he may develop and overcome them.

However, he is found to be not only conservative in the use of other forms of life, but scrupulously careful as to how they will increase and build up toward the one end, worldly prolongation. Seeing now, and knowing that man is the greatest and best of nature's work in the final prolongation of the earth.

Now, the question as to how man treats man becomes a matter of great importance in carrying out the perpetuation of the earth. Or at least its prolonging. What proves to be of benefit to the greater portion of mankind becomes the greatest benefit to the order of nature, or the law of our maker. Nature being benefited by the best there is in man will naturally suggest the way to carry out her work to the best end. Nature suggests to man to repel an attempt on life by any means possible. That being first law. A plan being laid by an enemy of society who of course is an assailant, nature suggests in the carrying out of the action a means of its overthrow. Thereby the enemy to so- 


\section{HEAT AND COLD.}

ciety becomes an aid in bringing conditions into being that will crush his order of beings. He brings into being a method whereby his class of man becomes extinct by his own movements. It being nature's law for survival of the fittest. Nature shows the way to rid the world of the rebels in the ranks of men tending to overthrow the natural laws.

If humanity is confronted with danger within itself, that same danger leads to the downfall of the main end of nature, worldly perpetuation. What is beneficial to the greater part of mankind proves to be the point of worldly accomplishment in the line of nature. Consequently, nature sometimes causes its best members punishment in order to make them concentrate their brains on the general welfare. And in so doing removes the corrupt means of punıshment from society that brought about such punishment. And in readjusting society to the proper channels toward worldly prolongation the injustice to the wronged one brings into being a method of crushing the injustice toward others. In other words, the means of carrying out the injustice becomes the means of crushing the power behind the injustice, because it brings into being a brain capable of weighing new impressions derived from the injustice inflicted. The brain weighing the new impressions becomes natures remedy in adjusting itself for the carrying out of the one end. Nature placing a party in the place who is capable of weighing impressions correctly. Therefore when we come to look over the ground nature tends to provide for the general wel- 


\section{MORALITY.}

fare of society in order that society may work to the better advantage, to the end of perpetuation of interchanging action. Liberation of the latent heat being a necessity in keeping the planet inhabitable. Man being compelled to work with union not antagonistic in order to arrive at the best end. Men who are dangerous to the general welfare being dangerous to the main end of nature. What more natural in the mind of the student of nature, than to say: Do unto others as you wish to be done by? If you do unto others as you wish to be done by, you will not assail anyone, and no one will have to repel the attack. If you do unto others as you wish to be done by you will never have any trouble with one of similar belief. The man you will have trouble with will be the man that claims especial privileges. Like the power who remains in seclusion and dares to give commands to humanity. The power that is a coward and will lay in wait to attack. That is the order of man society must be rid of in order to prosper.

Justice and honor have no secrets to keep. Justice fears not the light of reason. Justice seeks the light of question and loves it. Justice is one of the laws of the omnipotent of which we see and feel during every movement of our bodies. But behind the bulwarks of revelation-Bibular-we find the enemy to society. The cur who dares not face the light of day, who dare not appear before the balance that nature gave man, fearing that the true weight would be the result.

But instead, the real omnipotent powers of nature 


\section{HEAT AND COLD.}

in three forms, one matter, the other cold, and a third being heat. They fear not the light of reason, dawning within the mind of man. They gave to man the means he has of weighing his maker. They gave man the power that he may not be led astray by some tyrant who would assume the power monopoly earthly.

They not only manifest themselves to man's senses in one line but in all the senses he possesses. They are so thoroughly just as to render man incapable of being other than in contact with them. They, further than making man as a being, also made him out of the three properties. Each tending to do an equal part of the work in forming the man as well as all other forms of life.

So, poor little man, if you desire to worship your maker, do so by worshiping heat, cold and matter. But do not be foolish enough to look for revelation other than through your senses in the light of the period in which life is a fact in your case. After a thorough study of the passing events during your life within the properties, you may seek for conditions between the forces during any other period. Search for conditions between union of the trio of powers wherein the occurrence may come to pass. And by so doing you shall find truth, in or pertaining to any age or condition. But by all means obey the laws of nature as revealed to you through your senses by searching and examining her laws correctly.

Be not the slave of command from any source, only as revealed by the unchanging laws of nature. 


\section{MORALITY.}

But obey nature or suffer your own extinction by the blow that is self inflicted. Nature will not kill you, but instead let you kill yourself. Nature will show what is right and just, but will not command you to follow. If you follow you thrive. If you do not follow the laws, you crush yourself. Take your choice from what you see in nature as revealed by her unchanging laws. If you become a danger to society you will instruct society in a means of crushing you. Nature desires the perpetuation of society as a means to the greater end of unceasing action. An enemy to society is an enemy to nature. When you wrong society, the child of nature, you instruct the child in a means of crushing you. The child will study the case as revealed and will develop to the conditions and crush the being that threatens its wellbeing. Nature throughout all the avenues of action, acts the same, working to crush what is not beneficial to the principal end. Nature uses disregard for law in depraved particles or organisms formed by it, in suggesting to the better a means of crushing the kind. Therefore all the wrong order of life are simply self-condemned. They crush themselves by disobeying the true law of action or life as revealed to the senses through the life of each being.

Man may take matter while in the gaseous form and run down through all the changes coming to pass until its final extinction in worldly order by impact of a foreign body; or its worldly movement through space to unite with another body to give heat 


\section{HEAT AND COLD.}

and life elsewhere. And throughout all nature's action he will find that in no case has nature within her own laws condemned a being or product of her own make. She reveals her laws throughout every sense within each form of life she has molded, and left it to the person or manifestation to obey or disobey. Obeying, they all prosper. Disobeying, they each condemn themselves. The law of nature is, let the guilty crush himself. Nature has molded various forms, all working to the one end. The end being interchanging action or movement.

Man has distorted nature's law in the name of his maker; he attributes his making to corruption and follows along the line of his own choice. $\mathrm{He}$ goes through life oblivious of the maker being in contact with him, careless to her laws, and suffers accordingly.

Man may well, when standing on the threshold of existence surveying life with true knowledge of his maker as his portion, ask himself, Am I truly immortal? When you consider the grasp of his knowledge behind and before him.

He standing in the wake of millions of ages buried, yes, worlds by the million, gathered to eternity and transformed, to again take up the life giving condition. All this revealed to the man of a day, of an hour, in the wake of the change he surveys. What a grasp of knowledge is revealed to man who knows his maker. His maker having a law that never changes, so the being can look down the long trail of change and see himself in the elements. Seeing himself in 


\section{MORALITY.}

the elements, he can follow the maker's law through its course. Follow himself from the gases down to earth. When on earth he can look on and see his form develop from a beginning as a fermenting mass on the surface. He can watch it develop every avenue of circulation and excretion. See how and why each was a necessity. He can follow every change in the trials of his kind after development down through the ages. $\mathrm{He}$ can watch and see as if standing by, every cause leading up to his being manifest as an entity. He can see himself in the making before his parents were born, yes, millions of years before.

He can look into futurity ages, yes, millions of years. He can see worlds come and go in the future as in the past. And the only block that appears to stand in his way is the time of occurrence. He knows and positively, they will come and go. But as to time he cannot answer. There being so many causes leading up to continuance of a world when complied with. 'That he must ask himself will the laws be complied with? That is a question above his understanding. Man's awakening to the laws will continue life of a world millions of years. Will he waken to his true place in nature? That is the only enigma confronting man who knows the laws of nature. The real puzzle is his own kind. He knows his maker and what his maker will do, but he does not know man; he is the greatest puzzle in life. Let man know man and he becomes in himself omnipotent. When man shall have known his kind as well as he does his maker by a true knowl- 


\section{HEAT AND COLD.}

edge of the laws of heat and cold upon the particles of matter, then he can tell while yet living the day and date of any occurrence that will transpire throughout the future, as clearly as he can the past.

\section{THE LEGEND OF THE ARK.}

There is no question connected with bibluar writ more likely to have a foundation behind it in the form of truth than the story of the ark. Of course not as to actual size, and assuredly not as to date of occurrence. But as to the general occurrence of the inundation and consequent survival of people, by means of some boat or other means of being buoyed above the waters until their final subsidence.

The earth's crust renders conclusive proof of the foreign jolt from without. It also renders proof conclusive of the highest mountains being once ocean beds. Herein comes the proof of the legendary history which has undoubtedly been handed down from generation to generation; and of course corrupted by each particular translation from language to language.

Considering the means of man coming into existence by the cooling action within the particles of matter. Then the further fact of the earth's crust revealing the cause of past action. Then the necessary ages which had to pass over humanity prior to the catastrophe, we can see why that humanity must have developed so as to be able to calculate within minutes of the time of the fall of the body upon the earth. And 


\section{MORALITY.}

this calculation was perhaps years before the final collapse.

The impending danger being truly imminent and unavoidable tended to corrupt the mankind that next preceded the collapse. They, knowing they were doomed to perish at a given time, providing they lived during the period, tended to lessen their cares for morality and in fact every other earthly care dear to mankind. That condition having been the order of the times for perhaps generations before the final collapse. What more natural than the fall of man from his true place as a judge of worldly affairs? What more natural than the last generations before the collapse would look upon the man who passed judgment on the day and time of the occurrence as a God, especially when they, through utter disregard of life, had deprived themselves of development along the lines of knowledge capable of accounting for the occurrence?

What more natural than that the party calculating the time to a day, when seen from afar, would take on superhuman powers, when weighed in the minds of the ignorant? Of course the conditions would force man to deprive himself of education, knowing the doom was settling over all. What more natural than the man who calculated the day and date, and perhaps location of contact, in advance, may be one hundred years, knowing his figures to be true, would instruct his progeny to a means of saving themselves? He knowing beyond a doubt how heavy a jolt would be 


\section{HEAT AND COLD.}

the result of the distance. He knowing the amount of heat would be generated by the contact. He knowing that the heat would hold the water back a certain period of time. That it would not let it all run in fast enough to destroy the means of buoying up the living members of his family. They being able to land where the water had subsided from its old ocean bed? What more natural than that there were many families saved in the same way? Who were provided for by instructions in the same way, from men who were accomplished students of physics. Men who were not in any danger during their own period of allotted life. And were wise enough to know they were not, but concentrated their studies to the rendering their children aid in years after.

What better period in the face of the molding of matter into human forms by changing action and its capability of proof today, than the superstitious principle was instilled beyond the great catastrophe? In fact the condition of the earth's surface tends to prove that very little, and in fact none of the earth had arrived at sufficient heat since the catastrophe to generate new life of the man order. Consequently we have to consider man as the product from beyond the collapse. And not one ark, but many, conveyed the progenitors of our kind across the stormy issue, to again take up life under a superstitious heading. Who found the head, or fountain of life in the great astronomer or physicist from beyond the fall of the great body upon the earth. He knowing all about the fall 


\section{MORALITY.}

so long in advance must be the god of vengence when measured by distance. The greater the distance the greater his powers. People living at the time of collapse undoubtedly knew his standing as a man, but after the passage of the collapse he became powerful.

After the survivors having passed over the seas in their means of craft to again take up life under new conditions, it is quite likely that the parents of the growing children used the impact of the foreign body as a means of coercion when the children disobeyed. They telling the children that the great one from beyond would bring about a recurrence of the great catastrophe if they were further disobeyed. The parents not meaning to instill the belief within the youthful, other than a temporary means of bringing about order. But oblivious of the great effect it would have on mankind, it is very likely they instilled it in that simple way.

It is not at all likely that the people who survived the impact in the craft, one or many, had any belief of the occurrence occurring other than by the reduction in earthly temperature. Being well versed in the laws of nature, through the millions of years in the cooling process leading up to the loss of propelling power within the earth.

But as each succeeding generation used the same means in the bringing about of order, and while doing so neglecting the studies of nature, it gradually developed into a kind of religious belief. Children coming into the world generation after generation who never 


\section{HEAT AND COLD.}

heard the truth from beyond questioned. And in time the great being who was a child scarecrow in the beginning began to develop into a great man chastiser. The greater the distance that separated the big one from the children brought up in ignorance, the greater his powers became; until he held the finger of scorn over the human race.

Men coming and going, yes races of men coming into the world and retiring, who believed the great astronomer from beyond the impact to be a great god. Who grabbed worlds in his fist and threw them across space, tearing worlds and races into atoms. Until finally all mankind took to the belief in the great one of similar build and likeness that could extinguish worlds and suns in the twinkling of an eye. Who could grab up chunks of mud and blow breath into them and send them off in a gallop. Who could stand on tip toe and hang stars up on nails driven into nothingness. $\mathrm{He}$, the big one, standing on the earth and placing stars up on nails or limbs of sunflowers. The stars being little bits of matter millions, of times as large as the mound the great one stood on when lifting the load. But of course he would chastise the mound if it slipped from under him while he lifted up millions of times its weight.

Of course it was necessary that he would hang up millions of those little specks of light, each of which would make millions of earths. Always millions of greater objects paying tribute to the little ones. It would never do for the little ones to pay tribute to the 


\section{MORALITY.}

greater. Millions, yes billions, of those greater objects of light were placed for the benefit of our little mound. And the mound was made for our benefit. So, in the final summary, we find that he made all those great things for the ego. Lots of toys for the children. He was a great Santa Claus. And all humanity hung up their stockings for hundreds of thousands of years to get them filled by the great Santa Claus.

It is quite likely that throughout all this period of ignorance, during which the greater part of mankind believed in the astronomer being a god, that there were a few of the class who ruled who kept books or writings which revealed the truth. And it is very likely, judging from our earliest history dealing with gods, that some tyrannical king took command of the situation and gave his commands to all mankind. He knowing the fear which was instilled into the ignorant brains of his followers; then he felt that the time was ripe to assume the godship of all, thereby issued his commands. The first one of his commands revealing his human motives, all others weighing nothing in the face of the first. The first showing that he was a man, and the most tyrannical man that ever took breath through lung cells. His first command being the foundation to everything bad in man; it being the precedent to crime of every die; and he who set the precedent was the greatest criminal that ever lived. The most corrupt of men. 


\section{CHAPTER XXI}

\section{THE GOD OF THREE DIVINE PERSONS.}

From across the mountains of debris formed by the ages, buried in superstition, there dawns the truth, corrupted in the wiles of superstition. The great truth which was known to former ages as to the three powers of nature that constitute our maker; the powers of heat and cold upon the properties of matter.

They having been handed down to mankind after the great catastrophe of the foreign impact upon the earth. The truth being given to them in the form of distinctive names pertaining to heat, cold and matter. But, as the time passed over and languages changed and their meaning became corrupted. The three distinctive features remaining throughout all the ages as makers. But instead of being properties displayed in nature, they were interpreted from afar as being of own image and likeness, and consequently took the form of men. They took on peculiar powers not conforming to the correct powers displayed by the properties. Because they became individuals, they necessarily had to be jealous of their powers, lest they would be gobbled up by some other man. The qualities of men being to try and take from other men their rightful heritage. In consequence, when the powers were transformed into :nen from afar hey had to becom: 


\section{THREE DIVINE PERSONS.}

applicable to human nature in order to stand the test of time. In consequence: "Thou shalt have no other gods before me."

The foregoing was the most corrupt passage ever concocted by the lips of men. It tended to restrict man's research and consequent recovery from the depths to which he had fallen. His fear generated by the passage tended to keep him from finding his true maker. He not daring to search for his maker, lest he be punished everlastingly.

"Thou shalt not covet thy neighbor's wife, goods or man servant, or maid servant" is equally as inconsistent with true advancement. Because if a man covets anything he only desires same. If he does not go farther than desire he has done no harm to his neighbor. In fact he only has opened the avenue tc advancement by creating a desire to better himself. If he will strictly adhere to the command: "Do unto others as you wish to be done by," he needs no other command in morality.

All the others are superfluous. Some of them tending to carry out the teachings of the foregoing. While others tend to restrict popular advancement, by putting the ban on the clear exercise of the mind.

So we find that in the three divine persons making up of the godhead who was all powerful, nothing more than the corrupting of the truths revealed by former men who were acquainted with the true laws of heat and cold upon the particles of matter. They having given to their progeny who survived the inundation 


\section{HEAT AND COLD.}

occasioned by the impact of the foreign body, the truths as to powers of heat and cold upon particles of matter. Education being neglected after taking up life anew under different conditions, and as years passed the properties were interpreted as beings. The names of properties became names of powerful beings, jealous, tyrannical and unjust.

We will not conclude the chapter yet without bringing into play another proof of the knowledge beyond the age of superstition into which mankind has been imbedded; of man's place in nature in conserving worldly life by the sweat of his brow.

Nature proving in its unceasing laws that man must work to maintain heat in order to prolong his life and that of the earth. Now beyond the age of ignorance man knew that great truth. Men who came over the great catastrophe in the ark, or arks, as the case may be, were aware of the liberation of heat being a necessity in the prolongation of life, for man and earth. You will ask where we get the proof. Well, lest we hold you in suspense, will answer: In the burning of incense, making offerings to the angry god. In offering up life to assuage his great anger toward their kind.

Now, all of those peculiarities in the actions of the ignorant tend to prove that from beyond the fall of man by the impact, men were aware of liberation of heat being a necessity in prolonging life. The survivors of the catastrophe telling their progeny who lapsed into ignorance for want of education, that the 


\section{THREE DIVINE PERSONS.}

liberation of heat was a necessity in prolonging life for man, beast and planet.

Again we have proof in the worshipers of the sun, that the original life of man from beyond the impact was aware of the sun being a necessity in the maintenance of life. Because heat was truly necessary or life could not be. Yet if we had heat and no cold life could not be. If we had heat and cold and no matter life could not be. So take it all around, it becomes necessary to have all three in order that we may have life of our form.

When we come to trace the chastising properties allotted to the powers of gods as taught within the code of superstition, we cannot help but attribute its origin to the knowledge of the rules of nature in destroying the manifestations of life that are unworthy followers of its laws.

Nature's law, being the destruction of life by sickness and disease when its hygienic laws are not conformed with. Nature's law being work to maintain health. Nature's law being work to liberate heat. Nature's law being to preserve the best of kind. Survival of the fittest and best to the end of universal prolongation.

Taking this line of truths as a base, and believing that man, when advanced in the millions of years of life that preceded the fall of the foreign body, was thoroughly versed in same. Then it looks as if the chastisement of nature, as explained by former men, was transformed into instructions of men regarding 


\section{HEAT AND COLD.}

future punishment which would be the lot of erring beings. Of course the principal errors being to try and pry into nature to arrive at the true solution of the problem of life.

All the principal errors, such as hygienic cares, gluttony and other orders of same having a foundation in the teachings of the great god of our fathers. He being good enough to deny our forefathers the right to use the means given to us by our maker in the study of nature or our maker. He, the great tyrant, subjected men to trials which brought out the worst there was in man. He laid in ambush as a precedent for mankind in waylaying his kind. He dared not face man in the light of his knowledge of life if allowed to exercise same. Therefore he put the ban on man's inquiry as a means of reigning supreme throughout the ages. Until his means of government would bring about the untimely extinction of man. In the god of our forefathers as revealed by the ghostly misnomer of holy writ, we have the greatest enemy to mankind. Down with the tyrant, and long life to the dawning of the brighter day that is coming to the lot of man. Reason enlightening the world. Weighing all in the light of the passing action of nature, as revealed to all men, not to especial anomalies.

It is not at all possible that the astronomer who calculated day and date of the foreign impact left any instructions or commands tending to corrupt his progeny. But instead left a true catalogue of the laws of nature. It is very likely that he instructed the sur- 


\section{THREE DIVINE PERSONS.}

vivors as to the fundamental law of nature. That is, he left writings showing that there were three forces in nature, namely, heat, cold and matter, and that the three forces made everything. It is very likely that he also instructed them to observe all the laws of nature regarding health, etc. It is likely that he instructed them as to the fact that the liberation of heat was a necessity to the prolonging of life of their kind. It is quite likely that he instructed them as to the place they were wont to fulfil in nature, namely, to labor incessantly to liberate heat in order to continue life of the order as a means to preserve the little mound. It is quite likely that he also instructed them as to the dangers of doing wrong to an individual, showing them that wrong to an individual was wrong to the cause they all were wont to fulfil. Thereby he instructed, "Do unto others as you wish to be done by."

Some very unprincipled king, after the life was taken up anew after the impact and consequent inundation, undoubtedly turned all these good instructions into a form of personality, and took command as head of government. Then he transformed the instructions into a form of commandment that would subserve his desires as the leader of mankind. And fearing that man would seek him out and find that he was the most corrupt of men, he placed the ban on inquiry, and at the same time made a grounding precedent for all the corruption that has been the lot of all men who have come after him. 


\section{CHAPTER XXII}

\section{PRECEDENT.}

Under this heading all human action becomes measured; every government acts, or forces acts of its laws, to comply with some precedent; now, to inquire as to where the precedent is and should be is our object.

Where the precedent governing human action should be centered is in our maker; now to inquire as to whether it centers in the maker. We will attempt to show that our precedent does not lodge in the maker, but instead it abides in a dead, corrupt and tyrannical king of the ancient world.

Does the law as shown in this work admit of proof? Is it within the reach of any man to prove the truth of this law? If you can answer the foregoing queries in the affirmative, then you will know your maker, because you can prove who your maker is. Having proven your maker, then you will find that we have no law of precedent lodged in the maker; our precedents are lodged elsewhere.

Now, it behooves us to inquire where the precedent is anchored to, which governs human conduct within the laws of all nations. In elucidating this query we must resort to a number of questions and answers so as to make it clear and concise.

Human conduct being subject to precedent. Now, 


\section{PRECEDENT.}

where is the fundamental precedent governing human conduct by setting a lesson? Answer: In the god we love and follow. What precedent is most apparent to man and the easier to follow? Answer: Work in the dark; seclusion, ambush. How does god give man precedent to ambush fellowman? Answer: By his remaining in ambush while commanding men to follow precedent; to follow him. Why should he be a precedent in this case to man in ambushing fellowmen? Answer: Work in the dark, and work on men.

Now it is well to review the good god's commands. What precedent does the first commandment issued by the good god establish? Answer: It being "Thou shalt not bring false gods before me" establishes the precedent for man to follow, namely, hatred, jealousy and contempt for all but self throughout life. That command shows jealousy and hatred of all opposition, regardless of the justice of cause of same. That commandment is the foundation of everything bad in man. It leaves no room for inquiry into the truth of foundation of the god. It is tyrannical, unjust, jealous, criminal, prejudicial and felonious. Because it sets an example to all followers of precedent in human action; all loving to follow the example of the good god who could do no wrong. Following could be but proper, and in consequence the corruption of mankind.

It is not necessary to enlarge on the commandments as the first commandment forms a precedent that covers all human corruption. It is a precedent to steal what does not belong to you, because the god as- 


\section{HEAT AND COLD.}

sumes command and instructs to not question his prerogative. He has stolen the prerogative and fears question of same will reveal the truth of the theft. He establishes precedent to jealousy, hatred and contempt, by using the same passions as a means of coercion. He establishes a pearl of a harbor to crime of the darkest of every die by the precedent he establishes by laying behind a wall or rampart while ruling over mankind. He makes his precedent powerful and effective when weighed by men; men using the means of reason along the line of precedent can see no wrong in following in the footsteps of the mighty god.

In the other case, if man will follow precedent of the law that is truly his maker, and in doing so weigh all human action in the law that never fears question; in fact loves, stimulates question; thrives and expands and never lies when questioned. Then what will be the result on the human race? Answer: There never can be an injustice, because the law is unchangeable. What will be wrong at one time will be wrong always and forever. What will be right at one period of history will remain right throughout the broad range of eternity. The precedent will be revealed to every man in the light of his own life and will be as unchangeable as the unchangeable laws, which make same manifest to man's senses.

If man will anchor his laws down to the precedent set by nature, and follow it throughout, injustice can never be accomplished without being immediately revealed. Being revealed, nature's law is to punish. 


\section{PRECEDENT.}

Then nature will reveal a method of punishing the injustice when discovered. It cannot escape discovery by following nature's precedent; therefore an injustice will be impossible without paying the penalty.

Nature allows no one to keep a secret. Nature has no secrets. That being nature's first precedent. Second precedent revealed by nature being to use every means to maintain and follow the first precedent. What is the result of all secrets being revealed by nature leaving indelible lines telling the story of the act or action? Answer: It becomes impossible to do an injustice because it will be found out. Questioning the marks left of the work done will reveal the true mode and cause of action, when taken from every line of action that tends to converge on toward the point of accomplishment of the act. There being a thousand means of converging lines of inquiry toward the one point or act; and every one of these lines leaves a true story indelibly written and powerfully truthful. The summing up of all the converging lines in the language of nature's law leave truth unchangeable in the verdict.

But a mode of inquiry that will restrict question of any form strikes justice at the fountain head. Justice can never come to its own as long as question cannot be carried to any line that could possibly be brought to converge on the action. The question should not be prohibited from entangling the august being sitting in judgment, as that may be found to be one of the lines of converging truth revealing the true 


\section{HEAT AND COLD.}

story of the act or acts as the case may be. Nature having no secret and man should follow the precept throughout all his actions. Truth and justice will follow in the wake of taking a precedent from the law that made the man. It having no secrets and giving no commands other than punishing all wrongs; in consequence should be our precept of human action.

The law that made the man shows clearly where the man came from and where he is going; also what end he is here to accomplish. In these lines of truth it reveals another great truth to the man. This great truth being that man must do unto man as he wishes to be done by, in order to accomplish the best results toward the end he is wont to fulfil. Nature also reveals another truth in these same lines of truth which it has made clear to man. This truth being that man must resent an injustice to himself as a means to the greater end; that end being the end he is on earth to fulfil. If the injustice is such as would remove him from being, it is nature's law that he should remove the rebel in the ranks of men, as a means of preserving himself. In doing so nature has been doubly rewarded in the carrying out of her good work, because the dutiful follower of her laws has resented a mortal wrong and in doing so removed the greatest danger to nature in the working order of her laws toward the greater end. Not only has the law of survival of her best been accomplished but the danger to her system or government has been removed during the effort to carry out an injustice. In other words, the one who 


\section{PRECEDENT.}

resents the wrong and in doing so has to remove the cause of wrong, becomes doubly nature's most necessary preservative in carrying out her work of justice. 


\section{CHAPTER XXIII}

\section{CHRIST.}

Having dealt with life and the gods in general, and in consequence we find it not inappropriate to say a few words regarding the great exponent of the Christian teachings.

In passing judgment on the man Christ, we have to transport ourselves to the conditions that existed during his time, in order that we may not do the man an injustice. We must strike from consideration judgment conforming with the period in which we live, and act solely upon the evidence derived from authentic history as revealed to us today. Otherwise we would be judging him in the face of the enlightenment of the world during intermediate centuries. And the judgment would be in that case unjust. It will be equally necessary to consider the conditions that next preceded the conditions that brought into being a man of Christ's order. He having to develop with the conditions that made him in the true order of nature's law. Nature's law being, man develop to the conditions. Not conditions develop to the man. When we have grasped the conditions that next preceded Christ, and then collaborate the pre-existing conditions with the conditions in which he lived, then we shall know the man as if he lived with us today. Know him as 


\section{CHRIST.}

if we walked the streets of San Francisco with him every day for years.

Our reasons for knowing the pre-existing conditions, being to transfer ourselves to his time and take up the problems he had to deal with. We, in the face of knowing pre-existing conditions, become acquainted with the beliefs of our followers in case we take command of the conditions.

Now, that we have landed and safely on the scene to take up the problems confronting Christ. We have stretched before us a history of kings who were gods; gods all powerful. We have stretched before us in our history great gamesters who have done great deeds. The great gamesters are superhuman, nothing is impossible of accomplishment to these great beings. Our contemporaries are all believers in the genuineness of the gods that are pre-existing in our case. We can rally our followers in the defense of the gods. We cannot rally our contemporaries in defense of the nation. We cannot rally our contemporaries in defense of personal freedom or liberty. In our history there has often been mention made of great prophets who revealed the future. Prophets lived throughout thousands of years in our advance in existence. They were always revealing future actions which were to come to pass. They had a prophecy applicable to our conditions, and we could, any of us existing àpply ourselves to the conditions, providing we could show we were of a certain family. The progeny of the particular family 


\section{HEAT AND COLD.}

were all applicable in a way to the fulfillment of the prophecy.

Our country is overrun by hordes of strangers from a strange land; the strangers not only take valuables of every order from us all, but also take some of our loved and best neighbors and press them into slavery. The personnel of our nation and town are all subject to call at any time to serve the vilest of our enemies in the form of personal slaves. This condition does not apply to our town or nation, but is applicable to four-fifths of the known world. How shall we rally our forces to oppose the tyrants?' Will they take up arms against the Romans? No, never. The Romans are invincible; they have met and subdued the allied forces of nations on a thousand fields. When subdued in the field of action all are slaves who survive. Better lay low and let the Romans choose those they may, for slaves, and take a chance to be one of the fortunates in escaping the choice of the foe.

Bravo-Across the tide of history a star looms out in its resplendent glory. Liberty enlightening the world. Liberty-Liberty, in the prophecy we can see. A prophecy that will unite the human race. A prophecy that will find followers within the ranks of the enemy. We will whip the enemy within its own ranks. We will place her soldiers under our banner. We will unite all men beneath one head or god. We fill the prophecy and assume command; only stumbling block being that the prophecy foretells the birth from a virgin. Our mother is not a virgin. She is the dutiful 


\section{CHRIST.}

wife of our legal father and material father. Never mind, we will adjust the matter. Father in this 'case can be guardian, just neglecting the charms of a loving wife. Our cause is just.

Humanity is the stake. On the outcome of this issue remains the future welfare of mankind. Winning we have personal liberty for all men. Losing, we have slavery to nearly all mankind.

Christ takes command under the above conditions, as a political leader of his nation, and in fact a political leader of human liberty. He knows he is not a god and would gladly make it known to his followers if he dare do so. He knows he dare not make it known, because his cause, which is human liberty, will be lost when the truth is known. He must remain a representative of the prophecy in order to rally his followers, and to rally men who will come after he has passed away. He knows the battle is one of years, perhaps ages, but he knows it will win because the ranks of the enemy will fall in and take up the cause of the gods. The god belief being instilled in ages of progenitors, and the soldier will inherit the weakness.

Christ knew his maker; he knew that mankind was the means of carrying out a greater cause. He knew that union by just treatment to all was the law of the maker. He would gladly have rallied his followers under a truthful heading, but the time was not ripe for the carrying out of truth. Truth would have been shunned as the rattlesnake; it would have had no meaning within the times. Consequently whatever falsehood, al- 


\section{HEAT AND COLD.}

though knowingly, he had to resort to, becomes pardonable in the face of his object. And doubly more so when we consider that the amount of liberty his teachings brought about could not have been brought about under any other heading in the face of human conditions at the time. No man could have taken charge of the conditions with a twentieth century knowledge of man and nature, and done any better under like conditions within his followers.

Take it all around, Christ should be honored throughout the ages, as a great lover of mankind, a great apostle of human liberty. A great and powerful personality who could use the means that history had suggested in its former political safeguard for future eventualities. A safeguard or method of politics which had run through thousands of generations in preparing for future emergencies. Each generation making prophecies for the future that could be fulfilled by any one who read the history of the times, and cared to take the chance of applying himself to the prophecy.

As to a prophecy tending to prove that a man was a god, nothing could be more ridiculous to the man of the twentieth century. Because it would be impossible for a man to make a prophecy to-day and leave the same of record without having about six hundred millions of people fulfilling the prophecy when the time became ripe for the occurrence. Every man who read would aspire to its fulfillment, that is if there was anything to be gained by its fulfillment. If it was nothing more than to get before the public, they would aspire to its fulfill- 


\section{CHRIST.}

r.sent. But during Christ's time a prophecy had no means of solution to the ignorant, common-place people. But to men like Christ and thousands of his station in politics or priesthood, they could hardly repress their wonder at the utter ignorance of the rabble, in not comprehending such commonplace political moves.

As to the testimony of the apostles concerning the resurrection and the various other wonders which they attribute to the man Christ, nothing can be more condemnatory of the man's knowledge of humanity who exists to-day in accepting same as probable. The man who listens to the testimony of twelve buried men, or say one thousand men, who are buried beneath the debris of centuries, and accepts the same as truth, when the testimony is contrary to the laws of nature as revealed to him in the light of his own existence, he must be indeed very gullible and very ignorant of the nature of man of his own time. He certainly does not have character sufficient to inquire of his neighbors qualities not to say men buried beneath the debris of centuries. The man who cannot pass intelligent judgment on the characters of his contemporaries has no business inquiring into the personal characteristics of the buried ages. He is void of personality within himself, and should rest on the judgment of others. He is like the reed in the wind swaying with the greater influence brought to bear at the time. Let the wind change, he changes. A man who is willing to accept the judgment of twelve or twenty, or say one thousand men, testifying to an occurrence across the broad vista of buried ages. 


\section{HEAT AND COLD.}

He should have twelve twentieth century men sworn to take and weigh evidence, weighing him in the scale of life and death. He should then have impossible evidence, that is, physically impossible, brought to bear against him. He should sit and see in the light of his own knowledge the impossible accepted as possible by the men sworn to do justice.

Then he could reason thus: Twenty-five cents will buy sworn evidence in the light of my own personal knowledge, and within the range of all my five senses. They will swear to the impossible of accomplishment in the light of my own existence. They are good people, they have been schooled for thousands of years in the teachings of Christ. There are twelve passing judgment on me. There are many more giving evidence capable of proof, within the laws of nature as revealed; to be cruelly premeditatedly and criminally a most damnable lie.

Then the following cogitations should come to light in the mind of the man: If twelve will sit in judgment sworn, and lie in the light of my own knowledge, and my own life, then what class of man was the product of the dead ages? If many bore testimony against me that I knew to be false in the light of my own knowledge, and the light of my life; if nature's laws will prove the lie wherever it has been told, and is capable of being demonstrated on the stage, then what must I think of the ignorant beings who failed to have the truths of Christ's morals taught to them more than a few days; especially when the truth of their assertion is 


\section{CHRIST.}

physically impossible of accomplishment when subjected to the laws of nature as revealed to me in the light of my own existence?

In the light of the foregoing, the mind of the man will be thoroughly grilled in the knowledge of contemporaries. He certainly will know men as they are, not as they should be. Then in addition to the grilling should a man continue to observe men throughout his life, he will find that thousands, not twelves, will sell their own mother for the small compensation of one alcoholic stimulant. But should we be met with the explanation in defense of the apostles that they were better men than we have to-day. Then in answer we must ask, why is the testimony physically impossible within the laws of nature just the same as the lie that was given, which we can demonstrate to be a lie within the laws of nature in the case under our own observation while a living; breathing being? Further we will meet the problem on its true foundation, and ask why not back to the good conditions instead of continuing under teachings which have produced such horrible results after two thousand years of trial?

In final, we must back to the true judge and maker, nature, who made us, and take truth from where truth resides. Subject the evidence to the trials of nature when sitting in judgment of the case. If nature condemns by the unchanging laws, then condemnation is just. If nature condemns the parties placed to judge in the case, by showing their true worth, then place guilt where guilt belongs.' If nature proves the man placed 


\section{HEAT AND COLD.}

to judge a case, or twelve men placed to judge, to be violators of its laws by unjustly depriving an innocent man of his liberty or life, then subject the violators to the penalty they justly deserve. It is nature's law. Nature punishes the violator; the violator disobeys in depriving his fellow man the right of demonstration within the laws of nature, and he in turn becomes criminal in the judgment of nature, through depriving the innocent his rights within the true law. 


\section{CHAPTER XXIV}

\section{BIRD AND ANIMAL LIFE.}

On the mountain tops, or the highest points of earthly surface, during the cooling process, the first life of necessity had to appear, because the cooling was further advanced in like locations and caused the precipitation of the life-forming compound. The high points being exposed to the action of cold from every direction excepting from beneath, and in consequence arrived at the condition in the cooling process that would admit of the mingling of the organic compound that would build up life in advance of all other earthly locations.

The life of like locations had to be of an order conforming to short period of constant in the inhibited condition, or condition of incubation, because the cold being acting from all directions barring one, from below. In consequence, the fermenting period had to be that of hours when considered on extreme sharp points; likewise the particles of protoplasm precipitated under such conditions must have been minute, owing to the small amount of surface admitting of temperature conducive to its compounding. In consequence, minute forms of life. Insects of all orders, each conforming to the few minutes' difference in the action of different exposures to the action of cold; thereby differing in per- 


\section{HEAT AND COLD.}

iod of incubation, and in conformity, difference in species. The difference in the period of incubation marking the difference in species.

Life of the fly, bug and various other orders being the product of like conditions. They developing after a few hours into living, breathing, organic bodies; then the change had to be as rapid, owing to the conditions that made life possible of like order. The life had to develop means in a few hours, complying with the changes which were to come to pass. In consequence, the life of like locations taking in air and other properties through the pores and reducing same by the transforming mechanism of digestion from within into more minute particles. When reduced so as not to admit of further separation within the conditions in the organic system, then the particles were carried outward by the excretory passages of the body, or the pores. The cold having advanced so rapidly in like conditions of earth's surface as to congeal the particles exuded through the pores from within the organic body. Each succeeding lair of particles carried outward from the organic body being congealed on the outer portion of the next preceding lair. In consequence the organic body formed wings out of the exuded refuse from the body. The exuded particles being congealed on the outer portion of next preceding lair, and the same process continued until a fully developed pair of wings were the result of the portion of the body where the greater portions of bodily refuse were carried outward.

Portions of the body where the pores were more min- 


\section{BIRD AND ANIMAL LIFE.}

ute, the same process formed a class of smaller fins or a minute hairy covering, out of the exuded particles of refuse from the body which was carried outward. This hairy covering being also nature's process in working to perpetuate species. The cold continuing to increase, and in consequence the deposit carried outward continued to adhere to the outer portion of the next preceding lair. Thereby keeping the center open for the carriage of the off-fall from the organic system. The off-fall from the organic system building constantly on the outer surface of the body, and in so doing forming a covering for the body to keep it warm or conform to the change in nature passing in the interim.

This form of life had the pores of excretion more plentifully present in the portion of the body, where nature would develop by the waste matter from the system, a means of propelling life of the organic order. Nature using the waste matter to build up means of propelling the form of life upward and onward. Or more properly admitting of the propulsion of the form of life so as to admit of its transportation to points where change was not so extraordinary; during the interim the body was protected by a covering made of off-fall, same being hair, carried outward through minute pores provided by nature for the occasion:

Taking it all in all, we find nature provides means for securing all her works against any emergency.

Where the mountain tops had some body of earth so as to maintain temperature for a couple of weeks of constant, produced the bird life of various forms, each 


\section{HEAT AND COLD.}

form of species differing in conformity with the difference in location of posit of mineral properties, and minute differences of time of incubation. The bird life being subject to extreme changes and immediate also, owing to location; in consequence formed wings and bodily covering by the congealing of the exuded particles thrown off from the body through the pores. While the body was yet bare and being of such chemical construction as to cause the precipitation of particles from suspended matter, it took up moisture and other properties within the system. But temperature changing so quickly in like locations as to deprive the life of gaining sustenance by that means for any period of time.

In consequence the bird life began to feed on the insect life that preceded it. While feeding on the insect life, the cold became so extraordinary in short periods of time that the system in throwing off its exuded matter, caused the earliest exuded particles to congeal within the pores forming tubes. These tubes were kept open by the disintegration of particles into minute particles of matter, through the action of the digestive apparatuses from within generating heat to disperse outward. As each succeeding layer or load was carried outward by the deterging power generated from within it became congealed by the action of cold on the outer portion of the next preceding layer. This process continued until the stalk or bodies of quills was completed in the pores of the system.

After the stalk or quill was carried outward from the body so as to be denied aid in restraining 


\section{BIRD AND ANIMAL LIFE.}

the action of cold upon the particles of off-fall carried from the body. Then the cold acted on the reduced particles of matter, causing same when passing out of the quill to congregate into globular particles and adhere to each other by being acted on by heat in the interstices between the globular particles separated by the cold. This process continued by each succeeding posit of off-fall from the system, and interstices between globular posits were the means of further passages diagonally opposite to each other on the body of the quills. These again formed minute quills; and again were carried outward until globular posits were caused on each side by the action of cold on the deterged particles carried out of the system. In this manner the wings and feathers were produced by the action of cold upon the deterged particles of matter from the organic system. This process was nothing more than the condition made manifest, taking into consideration the immediate change coming to pass in conditions in like elevations where there was no body of earth to guarantee heat of a constant for any great period of time. This was nature's manner of providing for the continuation of species, let the conditions be what they may. The life coming into being under any conditions must conform to the conditions that was the cause of same coming into life.

The carnivorous life of practically all orders came into being farther down the mountain sides and slopes, where temperature could be maintained at a constant in periods conforming to their respective periods of gestation. 


\section{HEAT AND COLD.}

Carnivorous life being at time of birth a feeder on other life, had to conform to the conditions of temperature where it had to make its trips for food. In consequence the carnivorous life, although being the products of climates, not changeable in the extreme like mountain tops; still the life of that form had to develop so as to admit of its traveling and living on the mountain tops in quest of food.

In consequence life of the carnivorous order developed hair over the body by the congealing of the material carried out by the pores; through the action of cold on same. The life of like order being subject to extremes of climate through its quest for food on the mountain tops. The pores of the skin on like forms of life being small, and in consequence the hairy covering conformed to the capacity of the pores in carrying properties outward from the system. This form of life hunted the bird and other forms of life that preceded it on the higher points; also squirrels and minor forms of life adaptable to like abodes.

The domestic ox was the product of mountain valleys conforming in period of constant temperature very closely to the man. These valleys cooling much later than the mountainous districts. These valleys being situated near or in about the same elevation of the highlands conforming to the advent of man. They had greater bodies of minerals deposited owing to being the sinks or lowlands in proximity to the mountains, and in conformity the life developed in larger forms.

The domestic ox being a vegetarian from birth, 


\section{BIRD AND ANIMAL LIFE.}

had to make for the high points immediately after developing into a moving being so as to feed on the mosses or scums of detained waters which become deposited on the mountains during this period, or percolated through the rocks.

For the same reason, as other forms of life which generated a hairy covering to conform to perpetuation, he also had to develop the hairy covering by the congealing of the exuded particles of waste matter passing outward through the pores of his skin, through his being exposed to the rigors of changing climatic conditions.

He also formed his horns on the head by scratching against the rugged rocks trying to detach the particles of moss or scum which was the only form of vegetation conforming to the age and time. His gums being incapable of detaching the adhering moss, and in consequence he butted it off with his head. Each successive scratching and refreshing of the wound brought into existence the horny substance to grow on the head as a preservative of the head. It being nature's law to develop a means of perpetuation of every form of life and develop it in conformity with the conditions it has to contend with. This scratching process developed the horns in the form of a corn on your foot. Each successive abrasion caused the toughening of the sore, and made it more horny.

Even until to-day we see the same process going on in nature, where the shoe is so tight as to rub the hide and continue to do so, nature provides a horny substance 


\section{HEAT AND COLD.}

to grow in order to keep the abraiding source of trouble away from body. This horny substance when let alone will develop into a full-grown horn. That is when not detached by a knife or other instrument.

The domestic dog was the most intelligent of all the carnivorous animals, and came into being under about the same circumstances as the average animal of like order; but he being handicapped by not having the great strength of some of the greater animals, was forced to use his brain. The principal development in the brain of the dog being his development of the olfactory nerves, and in consequence he used his brain in hunting the minute scents that were buoyed by the waves of aerial properties. In consequence he was the first animal to make an incursion on the man with intent to feed on man. He having scented the man from afar off on the high plains when he was in the mountain districts. In consequence he made for the plains to feast on the new form of food. But when he arrived on the plains where he found man, he found a worthy rival both in brains and physical strength, and in consequence he was overpowered and domesticated by the man.

His domestication by man was a great aid to the man later in overcoming the other animals which later made an incursion on the man; the dog aiding the man to overcome the other animals. In doing so getting food for himself and his master.

The elephant and like animals were many years later coming into being, owing to having to await the 
cooling of extreme lowlands. The lowlands being extremely hot and being far above the temperature conducive to life when life was plentiful in the higher regions. The elephant developed without hair orving to coming into being where the particles of organic properties were plentiful in the air. The age of the elephant being so much later as to cause the remaining organic properties held in suspension to adhere around the lowlands or swamps that brought the elephant into being. Long periods in like conditions made life of long periods of gestation.

In all forms of life during the developing process, while developing the hairy or feathery outer covering by congealing of exuded particles from the body, the exuded particles were aided in forming into hair or feather by the deterged particles of poisonous properties denied entry into the system by the brain. These poisonous particles being precipitated by the temperature of the exuded particles, conforming to the temperature of precipitation of the poisonous particles which were heretofore held in suspension. When precipitated on the surface they were denied assimilation with the body of the man or animal by the brain passing judgment on same, condemning as injurious to the system. Then they were assimilated with the exuded particles which had caused their precipitation and in consequence aided in forming a feathery or hairy covering for the animal subjected to extremes of change.

Thereby we have no trouble in explaining the rea- 


\section{HEAT AND COLD.}

son why in the action of nature there was poisonous properties in the hair which could not be assimilated with the body, and thereby were not exuded from same. It not being necessary for all the properties making up hair to have been exuded from within in the working order of nature.

In the case of man he also conforms in showing how the order of nature never lies in its truthful lines of history which it leaves in its work for future questioning. The man has hair on his body in locations of surface where the outlines of body create a draft. These same portions of bodily surface have more pores and tend to carry off greater amounts of refuse from the body. The refuse being carried out in like locations and a draft being created owing to topographical conditions, as in planets and in consequence a lowering of temperature. Lowering of temperature necessitating the congealing of refuse matter. The congealing of refuse matter necessitating the precipitation of other properties in nature admitting of precipitation in the given temperature. In consequence, the poisonous particles going to make up the compound which we know as hair become assimilated with the exuded particles and form hair or feathers as the case may be.

In the case of man developing hair on the top of his head, it also admits of explanation as does all of nature's work whenever questioned in the language of her working order as observed to-day. The head having a heavy bone beneath the surface. This heavy bone having no flesh or fatty substance between it and the 


\section{BIRD AND ANIMAL LIFE.}

skin; in consequence no background to maintain heat. In consequence, a reduction in temperature of like surfaces. A reduction in temperature below the rest of body, while the temperature conforms to conditions of draining the system elsewhere; and in consequence the particles carried out of the system are caused to congeal on the surface of skin on the top of the head. When congealed they reduce the temperature further by getting further distant from the source of bodily heat. In consequence the precipitation of properties held in suspension admitting of precipitation in the given temperature; and union with the exuded particles. And thereby nature provides a covering so as to adjust that portion of the body to the conditions that become manifest.

It would be well for the reader to remember that the true law of life of any form will develop a style of life conforming with the temperature of constant. If the temperature of constant in a given part of the earth surface will conform in the low lands to the constant necessary to the production of man, then man will be the product of the given elevation. The ideas heretofore depicted only applied to the great continents in a general way. The temperature of constant is the given law, and seek for capabilities in the place questioned.

A low, level island having uniform temperature may arrive at the temperature conducive to life, and have the necessary properties in the mineral line to develop but few forms of life. The scárcity of mineral properties would render man the most likely style of 


\section{HEAT AND COLD.}

life, he being a product of long periods of temperature of constant, and a feeder on aerial properties for many years during the developing period. He would be the most likely form of life under the circumstances. There not being mineral enough to produce the other greater forms of life or means of sustenance.

It would be well to note that the conditions that made all hairy life was such as would render the pores practically always channels of excretion. The same can be said of all the fowls of the air which have feathery coverings. Their period of incubation or method of feeding reveals necessity for their coming into being in portions of surface of changing temperatures. The same can be said of the ox and the horse, they being vegetarians, and it being necessary for them to seek the coldest portion of earthly surface in order to get moss or other vegetable diet at the heated period in which they came into being.

In the case of the man, he changed the channels very often from exaction to excretion, owing to his method of deriving food from the mists by their means. 'And the temperature was constant in his locality for many years. In the case of the portions of his body which are covered by hair he nearly always used like portions of his body as avenues of excretion. The same will be found to be the truth until to-day. As like portions of his system seldom exact from without. They generally act as drainage avenues for the human body. 


\section{CHAPTER XXV}

\section{DANGERS TO THE HUMAN ORGANISM.}

Before closing this work, it would be well to consider the action of heat and cold in the light of their respective powers on the properties of matter, and weigh the effects on the human organism, and show the dangers to be encountered by abusing the true law of the forces.

When you place your hand into a basin of cold water the cold water causes the properties of matter making up your hand to contract. The contraction in the particles causes the sensation of cold. It is the union of particles in the hand. Change the hand into a basin of hot water and the heat expands the properties making up the hand. The expanding movement is recognizable as heat. You can immediately distinguish between heat and cold, because the particles of body change course of movement.

Now, if you are exposed to cold so as to freeze a member of your body, the frozen member should not be exposed to heat in any quantity. Why? Well, we shall explain the dangers from the foundation of the forces. Heat and cold always, when present in quantities, tend to demarkate lines of action of their respective forces; - herein lies the danger. When exposed to heat in quantity from without, it forms a zone of gravity between it 


\section{HEAT AND COLD.}

and the cold in quantity. The two forces tend to modify each other up to the line of demarkation, thereby imprisoning the cold within the member.

Again we will discuss another danger subject to the same law. On entering a very warm room after being exposed to severe cold, the cold having been sufficiently severe when without so as to penetrate the inner side of body, then the heat from outer surface of body tends to demarkate with the cold from within the body. In consequence, a zone of action becomes established between the two forces, thereby causing the congealing of blood within the inner surface of body. Cold being a uniting medium or force, acts on the particles of matter; heat contends on the outer surface for equal rights in action on the properties of matter. Result: the cold is forced to act in its own zone or within, and in consequence congeals the blood, causing congestion, and where the cold is sufficient, death will follow.

Remedy in like Cases.-When possible, there should be heat applied inward and cold without the body, thereby the heat from within would tend to drive outward, and the cold on outer surface would have a tendency to equilibrate, acting equal everywhere. The result would be the cold would unite in a zone outward, while heat would arrange in a zone inward. The reverse should be resorted to in the case of heat inward.

This zone is only temporary in the organic body; it is relative to the degree of constant heat applicable to the organism, but the aggression of either of the 


\section{DANGERS TO THE HUMAN ORGANISM.}

forces of heat or cold beyond the zone of demarkation established by nature in the contentions of the forces in the organism will tend to destroy the style of life. The zone of demarkation in each organism between heat and cold being relative, and the frontiers being extended by either force, and life will go out with the aggression.

The same can be said of planets; they demarkate lines of action between the two forces, but they are not eternal. When one force gains the ascendency in action on the planet, then the mode of life so applied will cease to be. So in either case man, planet or rose bush, when one of the forces, heat or cold, extends its power beyond its correlative in carrying on action, then the life of the order goes out with the aggression.

So we find that every move we make depends on heat and cold, which proves to be our creator, and how we observe the rules of our creator determines the period of life we will be wont to indure. 


\section{CHAPTER XXVI}

\section{DEATH.}

Death, when applied to the material man, is truly impossible, it being truly impossible to discover death in material. Material always changing, and consequently living in different forms.

But death when applied to the living, breathing phenomenon in the mutation of nature's conditions, it is possible and very probable, as it all depends on the man as to whether he lives or not, that is perpetually. He can live in the form of mind, but the mind must be anchored down to the unceasing, unchanging laws of eternity. He must think and act in the true order of the unchangeable. When passing out of this life by the mutation of the properties making up his earthly or material body, the mind anchored to the unceasing law of eternity will subvert to the unchangeable of which it lived a true representative. In the mutation of material properties the thoughts that dominate the body are the sole insight to its future action adown the tide of futurity. The mind that dwells on the unchangeable and loves its selection within the natural turn of change in all other properties, will live forever. It dwelling within the one and only property in nature that never changes, it will be eternal.

Should our world pass away adown the centuries 


\section{DEATH.}

yet unborn, yes, millions of worlds. Should humanity dominate the different worlds when temperature would admit of same, and in any and all of the periods of life adaptable to each and all of them, still the unchangeable laws will apply to action. So the mind that dwells on the unchangeable will be manifesting the unchangeable throughout all this grand process. Every property within nature tends to find its level. Like water in a tube always finding level. So with the departed mind when the material man shall have been liberated to again be transformed into nature's various properties. The mind will seek the level in conformity with its properties. If the properties of the mind prove unchangeable, then the mind is unchangeable. That is the mind living and loving the unchangeable while being in the form of man, when disintegrated it will revert to the unchangeable.

The unchangeable in life being the knowledge of the working laws of nature, the knowledge of what will happen or how it could happen from what we see in life in the passing action of the unchangeable laws. The knowledge of past, present and future from a study of what is transpiring within the range of the senses.

The mind that will dwell on the thought of a day which shall be shattered and changed with the conditions, shall live only until the mutation shall alter the order of the mode of thought. Then the ephemeral mind shall cease to be, to be replaced by another.

But the mind that dwells on the eternal laws of 


\section{HEAT AND COLD.}

nature that shifts down the ages will revert to the source of its power. It will be present in the unchangeable law which it was an earthly exponent while being in the mutation known as the man.

It cannot be possible that a mind dwelling in the form of man for a day, while surveying all nature and seeing his form of life compounded in the mists of the past ages, millions of years before he came into being, could lapse into nothingness. Especially when we consider the grasp of his knowledge while dwelling in the eternal action transmitted to his senses by nerves that are all pervading in their grasp. What renders it more unlikely that he should lapse into nothingness is the fact that the thoughts that are his, are the acts that are unavoidable millions of years hence, and they are unceasing and unchangeable, throughout all the grand purpose. We must conclude that the mind will live if it conforms to the true order of action, and that it will pass out of being or change form when not in conformity with the true law of nature.

But as to the perpetuation of form we must strike from consideration such question once and forever, as we have no other guide only what is revealed to us by the passing action of nature. Passing action showing change in every moving thing. Passing action showing change in every conceivable thing but the laws of action. The laws of action being the one and only property which appears to be unchangeable. The laws being ponderable to the mind - and not being ponderable to none other. 


\section{DEATH.}

Then we must infer that the home or level of the mind must be in the domain of the laws. When complying with the true and natural laws, then seeking their level, and becoming eternal as the eternal laws. When no-complying with nature's law, then being only a mutation assuming to law and going out of existence with death.

Let us diagnose this case, and then sentheses the same, and see if we cannot locate immortality in the mind, and do so beyond a reasonable doubt. We will simply tear down and build up, and see what result we derive from such action. First, the brain is made out of particles of matter. It is the seat of the mind. It has tentacles connecting with all matter. These tentacles carrying from any and all properties into which they come into contact with, and depositing same in the brain. The tentacles have direct connection with the two other properties of our maker, namely heat and cold. They also convey contact with the two power producing properties to the brain. The brain compounds the particle which has been conveyed for test with other properties within; in consequence, its makeup or quality has been determined by the brain. The result in this case must be law, and it is certainly mind. Now if this result is true to nature's law, which is immortal, then the mind that anchors down to nature is certainly immortal.

The result in the case leaves it to man to become immortal by the exercise of the powers given to him by nature. By conveying inward the appearance to the 


\section{HEAT AND COLD.}

brain on the part of tentacles of vision, we derive appearance of any and all things in nature. Lest appearance may foil us in deriving the truth of the particle in question as to appearance, we can resort to sound to further test the truth of the tentacles of vision. If we still remain in doubt as to its true place in nature, we can transfer inward by tentacles of taste. If the result fails to place the property as to law we can resort to smell, whose tentacles transfer inward. We can also test by touch. In each of these cases where we weigh particles of matter, the tentacles convey inward particles derived by the chemical action of the many forms of nerves. Each nerve having a tendency to take certain properties from the particle which has come into contact with same, the nerve conveying same inward to the brain. The brain forming five different compounds out of properties derived from the five senses; then balancing same and getting law as the result. The result being mind. Thereby we derive as a result of any and all tests in the action of determining matter, law, mind. Or law and mind proves out to be one and the same.

Consequently we must acknowledge that the mind that gets at nature's laws is as perpetual as nature's law in itself. It is within the reach of all, as we all have the means of weighing in five different ways. Matter is before us all, and we have the means to derive law from same. Deriving law from the action of nature's movement on matter places immortality in our grasp.

We will find also that when mind is the product of 


\section{DEATH.}

some great impostor's command from across the wake of ignorance, it is as long-lived as the impostor. The mind being law, and when anchored down to result of no standing, on temporary conclusion, must pass out with the means of its foundation. It cannot live beyond the standard of its control in action. When movement ceases to be within the standard of such law then all life built on the standard of foundation must cease to exist. Because it was nothing in the first place. It not being a living law of nature was only a temporary law or mind. Never having arrived at the goal of true law or mind. 


\section{CHAPTER XXVII}

\section{REVOLUTION OF THE MOON AROUND THE EARTH.}

The moon is a body formed around a gaseous nucleus and by that means becomes subject to the two forces of heat and cold in finding a medium in space. It will settle in conformity with its weight in relativity with the lifting power, or power that propels it. Only difference in the moon's action in space from that of the earth being that the moon is subject to two propelling forces in being held in place. And in addition it is subject to the intermediate relation between the two propelling forces and cold. Finding a dividing line between the lifting power two ways and cold making a line of demarkation with each force.

The earth being surrounded by a large body of air and in addition to the air the greater portion of the earth's surface being made up of water. And in consequence, the earth becomes a great medium for the deflecting of the rays of the sun. The earth's air and water make the earth, practically speaking, a young sun in the heavens sending out powerful buoyant rays. And in consequence, the earth acts as an additional sun in suspending the moon to a particular distance. The moon sinking in relativity with its weight to a medium where the cold, and the buoyant rays reflected from the 


\section{REVOLUTION OF MOON.}

earth are equal, and on that line of demarkation between the forces of cold and heat the moon moves about the earth.

The moon also sinks to the medium in relativity consistent with the gaseous center within it toward the sun, and in consequence it is like a balloon bobbing up and down on the line of demarkation of three forces.

When located beyond the earth or being in opposition or superior conjunction it is then shielded from the extreme rays of the sun by the earth and likewise deprived of the heating rays of the earth which cannot be deflected upon it owing to position. And in consequence it moves toward the earth, thereby proving why it nears to the earth during this portion of its trip around the earth. It also solidifys in the particles that constitute its make up by the congealing action of the undetered cold during the secluded period. Having gained in compression by the action of the cold during this period, it will be naturally heavier in relativity. And when the movements of the earth cause the earth to change position owing to the movement in its orbit, it leaves the moon falling toward the sun by the motion already under way. And the body of the moon gaining in weight all the time, owing to the action of the cold, causes it to move further on toward the sun. And at the same time the portion of the earth comes into play with the deflected rays of heat propelling at right angles upon the moon, thereby causing it to move in a kind of elongated ellipse toward the sun.

When this already accelerated motion gained 


\section{HEAT AND COLD.}

through the denial of the suspending power for a time and aided by the action of the cold, becomes expended, the moon has already passed on between the earth and sun. And during the period moving toward the sun it has lost a great part of its weight through the dispersing rays brought to bear from two sources, that is, earth and sun. It being lighter when between the earth and sun and the earth having a greater power through its being nearer and reflecting the rays of the sun back upon the moon, forces it farther toward the sun before it passes out of the influence of the tivo opposing forces.

When it passes out from between the two forces in opposition it has beyond it but the cold of space to deter it from soaring onward by the rays of the sun in propelling. It having been made much lighter, owing to the heat of the sun tending to expansion, and in addition to the heat of the sun, the heat reflected from the earth back onto the moon. And in consequence, it being far lower with relation to the sun than its weight in relativity would necessitate in the propelling it at a medium between heat and cold. Consequently it is driven away in a direct line. It being so light from being forced between two heating sources that it is carried away with great speed. Greater speed than at any other time during its course around the earth. When it passes out from the two opposing forces its weight being reduced, it does not take the course of an ellipse but is driven more direct.

By the time that the moon reaches its line of demarkation where heat and cold are equal, after passing 


\section{REVOLUTION OF MOON.}

out from the opposing forces of the earth and sun, it has already arrived at the point where the earth again shields it from the sun's rays as well as from the deflected rays from the earth. And in consequence, the moon begins another journey around the earth as before. And this same method is gone through for ages, or until the time when cold shall work its destruction.

The reason why the polar regions partially appear to us on the earth at certain times during the revolution of the moon about the earth can be attributed to the double sun power of propulsion of same in space. The earth acting as an extra sun by its reflecting power. It causes the moon to have two methods of revolution, one partially from pole to pole and the other from west to east as in the case of the earth. Having two suns requires two forms of revolution in order to preserve the body from annihilation by the dispersing of matter on one portion and piling up on another. If this dispersing did not destroy the equilibrium it would in time disperse from one side and pile up on the other until the gaseous center was liberated, and then the body would fall toward the point of least resistence. But the dispersal of the properties from one side and piling up on the other destroys the equilibrium and causes the body to turn about and then the properties are again dispersed and caused to precipitate on the lighter side again by the cold. The lighter side always turning to the cold after the equilibrium has been destroyed, and in consequence, a building up 


\section{HEAT AND COLD.}

again by the dispersed matter from the side exposed to the heat.

The moon moves from west to east, on its axis, by the action of the sun upon the body. While the moon is moving beyond the earth or during the period when it is situated more distant from the sun, it continues to pile up the particles of matter detached from the portion exposed to the action of the sun. All the while the cold precipitating the particles on the portion of the body directed away from the sun and the sun expanding the portion nearer to the same until the equilibrium has been destroyed and in the course of a revolution the displacement is sufficient to cause one revolution of the moon on its axis from west to east. The reason why the moon does not revolve faster can be attributed to the fact of there being very little elastic matter that can be carried from one side of the body to the other. The main factor in a body like the moon being the compression on the portion exposed to the cold, and the expansion on the side exposed to the action of the sun. Between the two forces it brings about sufficient weight in difference to cause it to revolve once in a revolution around the earth. Of course aided by what little moisture there is within the body being carried away and precipitated on the other side.

The polar revolution is brought about by the action of the earth reflecting the rays of the sun, and being in the premises like a young sun in the heavens with regard to action upon the particles of the moon. The earth bringing to bear great heat on the side ex- 


\section{REVOLUTION OF MOON.}

posed to the action of same causes the portion to expand which has been subjected to the rays. And the portion being deprived from heat pointing away to the cold becomes compressed and in consequence the moon is forced to revolve from north to south by the equilibrium being destroyed by the action of heat and cold. The heat of the earth being greater than the action of the sun direct upon the moon causes a revolution and a fraction during the period which the moon passes around the earth. Thereby accounting for the appearance of a portion of the polar regions of different dimensions during each revolution about the earth.

The revolution of the moon with regard to the earth proving that the earth's heat is superior with regard to the moon than the direct rays of the sun in the premises, causing a greater revolution on its northern axis than the sun upon the axis from west to east.

The action of the moon moving away from the earth and toward the sun when subjected to the two forces in opposition tend to add additional proof of the same fact, that the earth reflecting the rays of the sun reflect greater power within the distance than the sun does directly.

The reason why the moon rises far off toward the north during the winter months in northern latitudes can be conclusively attributed to the action of the sun. Owing to the sun being situated far off toward the south, it has its rays bearing obliquely or diagonally with regard to the northern hemisphere; and in consequence, it forces the moon off to the north by the driv- 


\section{HEAT AND COLD.}

ing power of same in the premises. It forces the moon to describe an elongated ellipse in the order of the diagonal power exercised by the sun in buoying the moon onward. The reason of the moon setting farther south than rising during this period can be attributed to the shift of the earth during the intervening hours.

The reason why the moon rises far off south during the summer months in northern latitudes can be attributed to the action of the sun with regard to southern latitudes. The sun bearing obliquely or diagonally with regard to the southern climes, and being a power to propel any and all things in nature. It naturally, when bearing on a large body like the moon, carries it off toward the south with regard to the earth. Again proving that no matter where the sun is located, its tendency is to drive away any body of matter that comes within its power of propulsion. Of course the limit of the driving power being reached when the cold, in compressing on matter, shall make the body equal to a given line of demarkation. Taking into consideration the gaseous center.

\section{PROBABLE DURATION OF THE MOON IN ITS VOYAGE AROUND THE EARTH, AND ITS FINAL CONSUMPTION \\ IN ADDING TO THE LIFE OF THE EARTH.}

The moon is one of nature's means of adding to the life of our planet. It is a reserve force for the rebuilding when life becomes almost extinct within the 


\section{REVOLUTION OF MOON.}

planet. When our planet gets frozen up so as to congeal all the aerial properties, as well as the material properties, then the earth will not reflect power of heat sufficient to suspend the moon. At that period the revolution of the earth will have slowed down so that the centrifugal power will be practically naught, and the moon will be precipitated upon the earth. The precipitation will cause a new period of life and warmth and add millions of years to the life of the earth. Heat being life and generation of great amount will bring about the mingling of the different properties within their respective temperatures of union. And in consequence there will be the compounding of organic life by the cooling order within the respective temperatures as in the former period of world building..

The precipitation of the moon will bring about all forms of life heretofore existent upon the earth, and in addition will be likely to bring into being new forms of life, owing to the mingling of properties existing in the properties of the moon that were heretofore nonexistant on the earth. The chemical properties existing being of an order that would mingle by chemical affinity within certain temperatures, forming into compounds that would exist in the organic order, or that of life in the animal form.

Having reviewed all nature in a cursory way, it now behooves us to ask a few more questions, and to answer the questions as suits our knowledge in the face of the evidence we have derived from study of same 


\section{HEAT AND COLD.}

through worldly action of same. Now, what is heat? What is cold? What is matter? Answer: In the face of evidence presented, heat is a mode of motion tending toward dispersal or separation. Cold is a mode of motion tending to union or aggregation. Matter is a property on which the two modes manifest themselves, or prove the capacity of their respective motions. The three combined make up our great and only power throughout all nature. Or to be more explicit, the three combined are, properly, nature. 


\section{CHAPTER XXVIII}

\section{INFINITY OF FOCI OR THE GENERATION OF SUNS.}

Different densities of the various gases we have in nature becomes the foundation on which we must construct our suns; or at least build from, in a way consistent with the peculiarities which we observe in the study of our sun. The law of foci tending to prove that where we have a transparent body of a given density, there will be a certain range of concentration applicable to the concentration of light and heat from some foreign source.

The difference we find in the density of the various gases that we find in nature, and the specific gravity being different in each case until conditions of temperature modify relationship in relativity. Hence, we find the means of accounting for the difference and division of the bodies of gases existing in the intersolar space. And divisions into zones acted upon by the source of heat generated within by the focused center of light. In each case a globular coating of a gas forming the inner belt or coat, covering next to the focused center of a given density; and again, the next zone bordering the outer portion of said lair of another gas, transparent and of a different density, and specific gravity, etc. Until a light which has been focused with- 


\section{HEAT AND COLD.}

in a center of globular gaseous medium forces the different gases to assume relative coats in conformity with the different coats around the human eye.

To take this problem up in the sense that will make our study clear to each reader, we will concentrate our example within the sphere of our sun; then we will carry the same process throughout all nature. We will make our sun the center of the universe for the sake of carrying out the law at the bottom of suns; while yet we know that our sun is only a minor sun. Yet in the premises it will be better to conclude from our sun as a point of getting at the bottom of the law of suns; it being easier for the mind to grasp the problem from the source within our range of vision.

Now our sun being a great body of burning properties of matter at the beginning of things, it has a power to reduce all matter into minute particles so as to feed the flame. Its powers being always to disperse by reducing matter into more minute forms, as the particles of matter become nearer to the center of power. In each case the particles reduced by proximity being lifted by the propelling power and thrown against the next range of particles of greater bodily dimensions which range outside of the given range of reduction by the action of heat. These particles being impinged against particles of greater dimensions whose weight are greater, and in consequence the lighter or reduced particles are forced toward the power or source of heat, being the sun. This process continues by reducing particles of matter from the 


\section{GENERATION OF SUN.}

surfaces of earthly bodies, throughout all space, until particles by relativity in weights battering against bodies of greater weights are forced into the flame by impinging against other properties of greater bodily proportions which cannot be reduced in the range of temperature which exists at the given distance from heat. This is the means of feeding the flame of the suns. Always driving away and reducing properties into more minute particles until they become so reduced as to be forced into the flame by impinging against greater bodies which cannot be displaced by the jolt.

Now that we have derived the means of feeding the flame without changing the law of heat as observed daily within our surroundings, we will further enlarge on the same law and find whąt results we can derive from same in generating other suns. The sun, after being burning and dispersing matter for millions of years, tends to range matter into zones or coats around the central medium. All properties of matter having a different power of heat necessary to the arrival to a given zone. Heat that would bring properties of one order to the edge of the sun would leave other properties off in the distance; therefore, properties when continued to be reduced throughout millions of years become aligned into zones conforming to temperature in relativity.

This process goes on throughout millions of years, and overhauls all matter by each succeeding property being used for pabulum to feed the flame as 


\section{HEAT AND COLD.}

it becomes reduced by coming or sinking nearer to the source of heat. When one property has been all used up and driven off in the distance by or in the form of sunlight, then the next property ranging in relativity in reducing capability comes in to act as food for the flame.

But throughout all this grand purpose there remains various zones of properties of matter reduced by heat into zones around the sun, and thereby forming coats like the coats formed around the human eye. These coats of transparent matter ranging within their range of different density and specific gravity in relativity, with regard to the sun. In consequence, they act as means of concentrating the power from within the center of force, or converging same through their respective differences in relativity to some point in the distant space.

When this force of the sun becomes reflected on the gaseous medium in intersolar space it acts the same way as the parent sun, by beginning to feed itself from the bodies of properties of matter. The more reduced particles being thrown into the flame by the action of the power of separation or dispersal occasioned on the properties of matter. In consequence, the new sun begins as a focused center of light, but immediately begins to feed on the properties of matter. Heat being so great in the focused center as to begin acting on matter by separation of same into minute particles in conformity with the heat generated in the given center or focus. 


\section{GENERATION OF SUN.}

This grand process goes on to infinity, each sun forming lairs of reduced matter into relative zones conforming to specific gravity in relativity with the power of reduction in different temperatures of reduction of the different properties of matter. A sun prior to its extinction converging its lines of light so as to bring into being another sun, through the ranging of matter around the same in zones of relativity in its dispersing properties on matter.

\section{DURATION OF SUNS.}

Every law of nature tends to prove that whether or not suns are burning bodies within themselves, or have the reflecting power exercised through the medium of a gaseous substance, still they work their own destruction by repelling all the time.

Should our sun have the reflecting power of some other sun, it would, in conformity with nature's law on the reduction of the properties of matter, by dispersing same into minute fragments, bring about its own annihilation. Every different property in nature having a different temperature of separation into particles of a given weight or dimension, and in consequence, its extinction.

Should our sun be a burning body of particles of matter, it would still work its own ruin by the same laws of matter, owing to having so varied a number of properties in nature and all of a different specific gravity and of a different temperature of arrival at a given weight by its separation into particles. 


\section{HEAT AND COLD.}

All it requires in the extinguishing of either form of sun is for the sun to continue to disperse the particles of matter until some form of matter that is incombustible becomes the dominant factor in the zone next to the source of greatest heat; that is to say, next to the sun. When that form of matter becomes reduced in sufficient quantity to act as a means of stopping the next grade of matter reducible to minute particles from contact with the required heat to cause combustion, then matter begins to form in zones in conformity with its specific gravity in relativity. Finally there appears a grade of matter which forms in a zone, and it being of an opaque character, and the temperature not being sufficient within the zone to cause combustion, the particles of matter between it and the sun being non-combustible and when buoyed away by the power of the sun in repelling becomes again thrown back on the sun, owing to least resistance. In that way the sun is denied any further food for the flame. Being denied any further food for the flame, then the body begins to cool and new zones continue to form in the particles of matter beyond the opaque property of matter until the sun is cut off from the outside world; and in consequence continues to cool.

The tendency throughout all nature being for the sun to disperse all matter. What more natural than that the particle in nature most reducible at the greatest heat to minute fineness, would continue to be thrown back on the source of power propelling it, especially when it is forced out against bodies of greater weight? 


\section{GENERATION OF SUN.}

Not unlike throwing a stone against the earth, causing it to go backward toward the cause or force sending it, owing to not being able to displace the world by the jolt.

Then what more natural than that in time the next reducible property in nature would finally arraign itself in a zone next the property having the greatest reducing power in the order of heat; and so on to infinity until the sun became enveloped and denied any more pabulum to feed upon.

The very fact of nature having all these properties that will render the destruction of suns a fact beyond question lends great evidence of the fact of suns being generated by the law of foci. Because in the order of foci the generation of new suns are insured continually, one building another before it becomes extinct.

As to the destruction of suns by that means we need not leave our planet to find proof of the fact. All we require is to study the action of the sun upon the particles of matter here on our planet. Then to carry out the destruction of the sun, all we require is to study the requisite amount of heat needed in the dispersal of the fragments of matter here on earth to a given fineness of weight and dimension. No man can possibly be so dull of comprehension as to doubt for one minute that in the course of millions of years the particles of matter most reducible will find a zone within the given fineness. And that kind of matter being reducible to units below other properties of matter will continually 


\section{HEAT AND COLD.}

be forced back against the source of power tending to throw it away.

The fact of a property being aligned in nature against the sun so reduced to minute particles as to deny the contact of other properties would not necessarily work the destruction of the sun if it would be the only property so aligned. Because motion is heat, and the motion would tend to keep up the heat if it was not for the other properties that began to align themselves in the order of their respective weights in relativity. Until finally opaque substances began to form and each succeeding zone would be colder until the body as a whole was required to cool off as a mass.

The sun in our planetary system renders evidence of different envelops forming around the sun, and it is nothing more than natural that they should form in conformity with the laws of matter. The sun spots being in that case but the earliest manifestation of the gathering of the opaque properties tending to the extinction of our sun. So again we have to concede that nature's laws here beneath our gaze never lie when questioned.

Until the matter of a given weight becomes plentiful enough to deny the contact of other properties, with the sun, it will continue to feed itself by driving the particles of matter next to the heated source against other properties of minute dimensions, causing the other properties to be forced back into the flame like water from a pond when jolted by a large stone. When the properties become reduced to minute units by a 


\section{GENERATION OF SUN.}

burning process, they are driven away in the form of sunlight. Thereby the body of the sun becomes replenished. Feeding the flame by particles jolted, by the reduced particles in the form of sunlight. Thereby the particles of burned matter inflicting a jolt to the other properties caused the same to bound into the flame. But all the while particles of matter are being reduced to such minute particles as to return with the matter acting along the lines of least resistance, and are continually accumulating in body to some day destroy the source of power, or the sun.

The sun, when it gets enveloped with heavy zones of opaque matter so as to deny the escape of particles of reduced matter will begin to cool. After falling in temperature the gases enveloping same will begin to condense around the sun. The property capable of condensing into a liquid solid character will form the inner crust. Then each succeeding property will settle in conformity to its temperature of precipitation until it becomes a planetary body. In the course of millions of years, perhaps billions, it will cool sufficiently to admit of the precipitation of the life-producing compound. Then when the period of constant has been reached conducive to the building of man, he shall become an inhabitant of the surface of the planet cooled from the properties settling around the sun.

The center of the sun throughout all this cooling process being the most minute particles of matter. The most reducible in all nature as to attenuation; and in consequence, when imprisoned by the settling belts of 


\section{HEAT AND COLD.}

matter in conformity with their temperatures in relativity, it will form a very attenuated gas when closed away from chance of escape, or smothered. In consequence it will buoy the body as a whole to some medium between another sun and the extremes of cold, and there continue to revolve by the action of cold and heat on the particles of plastic matter composing the outer borders. This revolution will be very speedy during the heated period, because the gases will expand more when acted on by heat, and contract more when exposed to cold. Thereby the expansion on one border of the body and the contraction on the other will cause great displacement in outlines, causing great disturbance in equilibrium; and in consequence, great speed in revolution on its axis.

As it begins to cool, less displacement in outlines by contraction and expansion; and in consequence, slower movement. The orbital movement, while very hot and gaseous, will be very slow, owing to displacement from polar regions to polar regions transforming the power into work. 'The distance from the sun which buoys it will be very great, owing to the great nucleus and the heated condition of the crust or forming body. 


\section{CHAPTER XXIX}

\section{CONFORMATION OF PLANETS.}

The law of solar propulsion, when applied to planets, varies with the age of planets. The older planets being more compressed by the action of cold upon the particles of matter, and in consequence settle nearer to the sun. They settle nearer to the sun owing to being heavier in relativity. That is, taking into consideration the water or aerial properties which go with each planet.

Of course the outer appearance, or extent of surface, of a planet would not be a safe mode of weighing a planet so as to determine whether it was heavier in relativity; because the gaseous nucleus may be larger in one than the other, and again, the aerial body surrounding one may be greater than another. The better mode of measurement would be to judge by the state of compression apparent from outer surface. The surface being a clearer index to its age, because it would show the state of compression Mercury being and appearing the more compressed of the planets, and in consequence has settled nearer to the sun. Venus being the second in age, compression and appearance, has settled nearer than the earth.

The earth being third in age and compression, but having life of various orders tending to transform 


\section{HEAT AND COLD.}

latent heat into the active form; and in consequence stands a fair show of out living other planets, providing that the life here existing will out-act life of other planets in liberating same. Knowing we have the means and intelligence capable of the liberation of heat, and not being sure of other planets; in consequence we have reason to believe our planet will endure longer than planets much younger in the scale of compression, or situated beyond our earth with regard to solar propagation.

Mars being fourth in the range of compression, and in consequence is situated beyond the earth or farther from the sun. It is quite likely that Mars will eventually settle down so as to replace our moon when the moon has been precipitated upon the earth at some future time when it has cooled so as not to be able to propel same. The moon having been precipitated as a former body has, at some remote period, already done, it will generate more heat and enlarge the gaseous medium surrounding the earth. It will bring into being new forms of life along with all the old forms, which will come into being under the same temperatures. Mars will have cooled so as to replace the moon by having settled nearer to the earth. The earth's influence will be greater, owing to having a greater gaseous medium surrounding same. It will reflect greater amounts of heat which will be impinged against it by the sun. This reflected heat will have influence as far as the range in the heavens where Mars has settled to by the compression within its particles. 


\section{CONFORMATION OF PLANETS.}

This reflected heat from the earth will change the motion of Mars so as to render it subject to two forces, as does the moon today. The sun acting directly by propelling and the earth reflecting the rays of the sun and also propelling. Thereby the earth's propelling power will cause by its action on the particles of Mars, a rolling about which will keep it within the two influences, until some later day when the earth has cooled again so as to be unable to propel Mars. Then Mars will be precipitated upon the earth and generate a long period of life and warmth.

Jupiter is propelled beyond Mars by its own heat, aided by the heat of the sun. The planet Jupiter being buoyed more by its own heat, may be explained thus: Heat being expansion or motion, more heat being more expansion and motion; in consequence the whole body as a unit is lighter in relativity; and the minor power in the premises has a greater effect upon a larger body of light weight. Jupiter appears to be in the making, and it may be millions of years before it will have cooled sufficiently to admit of the precipitation of the organic compound which will ferment into life. But whenever it shall have cooled sufficiently to admit of the precipitation of the organic compound going toward making of life, then it will have settled much nearer to the sun, owing to the compression in the particles of matter which constitute its formation. It having settled within that range, then life will be necessary, providing the properties are present in the formation. 


\section{HEAT AND COLD.}

Saturn is situated farther away from the sun than Jupiter, and is sixth in range from the sun. Like Jupiter, it is propelled more by the heat from within than from the sun. It, like Jupiter, will be millions of years in the cooling process before it will admit of life, it being too hot to admit of the precipitation of the organic compound. When it shall have cooled sufficiently to admit of the precipitation of the organic compound it will have settled nearer to the sun, owing to the compression of the particles which make up its structure. The organic compound could not possibly be precipitated upon either of these two planets. If it was thrown on the surface of the planets or either of the two in chunks as large as the moon, it would immediately be reduced to gases by the heat generated from within the planet. It would remain in the gases until a temperature arrived which would be conducive to its precipitation.

The same law of time and compression will apply to Uranous and Neptune, with regard to settling nearer to the sun and to the generation of life on their respective surfaces.'

All planets yet in the forming process move faster on their axes, owing to the great expansion in the properties of matter forming same. The properties being greatly expanded by the heated condition of same, and in consequence are held a great distance away from the sun through its action in lifting on the unit. Cold being extreme condenses on the outer borders and the sun relaxes on the exposed borders of same; this process 


\section{CONFORMATION OF PLANETS.}

causing the revolution. The relaxation being great on the properties when exposed to the sun and aided by heat from within. The contraction being very great on the outer surface of body when directed away from the sun, owing to the medium being very plastic or in an early condition of compression. In consequence, great displacement of outlines or equilibrium creating movement. As the property expands and contracts, so does the movement increase. Reduction in expansion and contraction reduces movement. Thereby we note that a colder body situated nearer to the source of heat and farther away from extreme cold must move slow on its axis. The sun being incapable of displacing much of the solid outlines by dispersal; and cold having a solid body to compress while not being extremely applied; in consequence, reduction of motion or speed on the axis.

As bodies get colder or more compressed, they slow down on their axis, as shown by the above law. As each body gets colder or more compressed, it will move faster in its orbit by the same law. The body being more compressed, and at the same time being situated nearer to the source of heat, which is a lifting power, then the sun lifting on the body as a whole, owing to having a solid which will not immediately disperse before the rays; in consequence, the lifting power manifests itself on the whole body and moves the whole body at once.

This law on the revolving of all the bodies in space, both in the orbits and on the axes, will be found 


\section{HEAT AND COLD.}

to conform throughout all space with the already accepted truths as to movements of planets, orbital and axial.

This law again proves how conclusively the sun acts throughout all space as a driving, lifting and separating power, just as it does in our simplest observations earthly. Just as it expands the finger so as. to render the finger ring tight on a hot day.

The planets near the sun are the solidest from exterior appearance, and they should be in order that the sun could move them faster in the orbit. They should be more solid in order that the displacement by the sun during exposure, and precipitation by cold during exposure to cold, would be less, so as to reduce revolution on the axis. In each case they do conform.

The cause of planets sinking nearer to the sun can be explained by loss of heat within the unit. Loss of heat within the unit means compression. Compression means greater weight in relativity. Again they all conform.

A body being more solid nearer to the sun as proved by compression, and thereby must be less dispersable by a given amount of heat; in consequence, great orbital movement through action on the body as a unit.

The same law proves by compression and proximity being the same, that the reduction in the dispersing of matter and likewise of the power of possible compression on particles of same, must inevitably lower the axial movement of the body. Thereby we get a 


\section{CONFORMATION OF PLANETS.}

result conforming with all the truths as to movements of bodies in space. The law accounts for every movement and variation throughout space. And what is the law? Answer: Heat and cold acting upon the particles of matter; just as your five-year-old daughter would observe in her movements about the house. Each one of these forces acting just as she sees them, nothing less, nothing more.

There is no variation in the planetary heavens which will not admit of proof by the study of heat as we know it, and cold as we know it, without trading off either of the powers exhibited before our eyes.

Where one planet tends to move off toward another in the distance in the heavens and has heretofore been attributed to attraction, can be conclusively explained by the lessening of the action of cold from the opposing side from sun. The lessening of the cold on the particles of matter that constitute the planet on the outer border will render the action of the sun greater in relativity, and in consequence the sun will lift or drive the body as a unit farther away. Because the planet was moving in a line of demarkation between two extremes, one heat and the other cold, reduction of the compressing power of one renders the other greater in the premises. Consequently the heat of the sun buoying the planet causes it to move avay from the sun.

\section{DURATION OF PLANETS.}

When planets sink toward the sun by compression within the particles of the unit and have no satellite left 


\section{HEAT AND COLD.}

to lend additional life to the body by impact against same, then the planet will continue to sink toward the sun until the sun will generate heat enough to separate faster on the exposed side toward the heat than the range of cold will admit of compression on the outer side. Consequently that method will discontinue the revolution. The detached particles being detached faster than the degree of cold will admit of precipitation on the outer side. Consequently, no revolution. No revolution means liberation of the gaseous center and a hasty trip into heat sufficient to burn the body into the gaseous form again.

So we have one means of delaying the burning either by impact of the moon or by impact of Mars; or last, but not least, the hasty trip into the regions near the sun where we will be reduced to gases. This method remains in liberating stored heat on the planet and thereby causing the earth to continue warm, and fast enough on its axis to protect itself from foreign bodies. The speed it has at present on its axis is a safe guard from foreign invasion. The heat it has in the gaseous medium which is liberated heat also tends to reflect the heat of the sun and suspend other bodies like the moon which desire to fall on the earth. The heat in the liberated form in the body of gases around the earth also tends to suspend the earth at a great distance from the sun. By liberating the bodies of gases or properties of matter which are solidifying and causing the work of compression to be gone over again and i 


\section{CONFORMATION OF PLANETS.}

again, we can prolong the life of our planet indefinitely.

And that is the object our maker had in view in molding the man, simply to work to the end he has of late years unconsciously striven toward. The maker made man as a means to an end. The end was continuation of life of his kind, as a means toward the greater purpose. The greater purpose was continued life of the planet. Perpetuation of man as a means to perpetuate the planet he lives on by keeping the comprestion delayed. Compression meaning death to the planet and man. 


\section{CHAPTER XXX}

\section{DARWINISM.}

Darwin did more to disprove his hypothesis of evolution or natural selection, having built great forms of organic properties out of nothing, than all the leaders in thought during the last generation. It is very questionable as to whether he ever had any idea of advancing the hypothesis other than as a means to a further end. But on finding that the world accepted, or partially accepted, the same as truth, he naturally acquiesced in the decision.

In the face of the preponderance of evidence he brought to bear in downing his hypothesis, it does noi look reasonable that he himself believed in it. He undoubtedly had but one object in view throughout his work, namely, to display the wonders of nature which were revealed to him in the light of his own senses. The wonders, which through his eyes were revealed as working to one end. That end he knew not. The cause he knew not. Throughout his work he repeatedly confirms that judgment as passed upon him and his works.

He undoubtedly was a great judge of mankind as well as of all organic life, in the light of the time in which he lived. That is, he observed all their peculiarities, habits, modes of life, action and thought. 


\section{DARWINISM.}

But cause or law at the base of all this grand purpose was more mysterious than he even acknowledges. But he thoroughly acknowledges lack of knowledge beyond his observations in habits or structure of beings questioned. These observations lead him to multiply the absurdity of his want of knowledge as to law, by grasping for a means of carrying his reader along through his work. The means was based on the weakness, which he knew all too well to be the dominating feature in the personality of each and all of his contemporaries. He looked for some means which he could place in the foreground, something mysterious, because the life of his time all looked into mystery for knowledge. He continued this mystery in the foreground, while displaying a keen knowledge throughout all the work of all the animal and other organic properties as revealed to him by study and observation. He dare not drop the mystery in the face of the period in which he lived, or he would not be able to carry the reader along through his exposition of the peculiarities revealed in the working order of organic nature. In consequence we find that the hypothesis was nothing more than a bait for the gullible. It had no meaning to the writer other than a means of carrying out the greater cause; that is, displaying the mysteries that were truly mysterious to the writer. He choked the other weaklings with one mystery that they may inquire into the great mystery which was confronting himself. The why, whence and whither?

His work may be summed up in a few words, that 


\section{HEAT AND COLD.}

is, the portion leading to the establishment of the hypothesis. First, he never brought any proof to bear other than within a given species, tending to show crossing or change. Pigeons were intermarrying and in consequence bettering the grade. Different families of men intermarrying with each other, and in consequence bettering the species. Dogs crossing with dogs, and in consequence bettering the same. Horses crossing and cattle crossing, but in each case crossing with their own species. In all historic periods or geologic periods he could not bring a case to bear where one form of life could combine with another, and after combining become prolific. He proves conclusively that it did not occur by reviewing all history both earthly and human.

But while he proves the absurdity of even chance crossings, he brings into play the near resemblance of properties in organic nature, taken as a chain from smaller to higher grades. From the smaller forms linking on close to the next higher grade until the whole reveals one continuous change from bottom to top. One property gradually bordering onto the other. In this he has truly discovered that the whole of nature was made by one law. But the law he knew not, and acknowledges he knew not in his work, while holding the hypothetical object in the foreground as a means of carrying the mysterious all-devouring being onward, by the pabulum he tends to reveal which will assuage its gluttony.

Should Darwin have actually been convinced of 


\section{DARWINISM.}

the truth of the hypothesis which he carried as a subject in his works, then the best we can say,for him is that he lost the object of his subject, in his display of knowledge of the natural inclinations of the various organisms which he described. His love of display of the knowledge of their respective characteristics buried the intended object, which he hoped to attain, and in consequence he acquired the end which was not his goal, in the outlining of his works. Or in other words, he deceived himself by leading out to one end and deriving the opposite.

The author of this work feels in no way indebted to Darwin for what aid he may be in proving the truth of his deductions, because he never read Darivin's wyork until he began the chapter entitled "Darwinism," but since the reading of his works he finds that Darwin's researches will be a great aid to the searcher for light on the subject heretofore outlined in this work. Darwinism being the last chapter written in this work, and no alterations being necessary from the reading and commenting on same.

Darwin has repeatedly run amuck with problems which he had to confound with the most nonsensical modes of explanation, and in each and all of such problems which turned his blood cold in his veins, are in conformity with the true law of nature. He not being familiar with the fundamental law, while trying to place a false law at the bottom or foundation of nature; and in consequence ran amuck with the true order of nature's work during his researches. Consequently he 


\section{HEAT AND COLD.}

had to resort to interminable explanations and conjectures in order to even make it appear as though there may be a foundation to link onto, so as to draw out into a weak hypothesis.

The author will recommend the works of Darwin, that is the part pertaining to his researches into animal distribution as well as vegetable distribution throughout the surface of the earth. And while recommending same, will call the reader's notice to the animal and vegetable distribution as encountered by Darwin, and the most difficult problems which he came in contact with conforming with true law. Then he will ask the reader if he can find any place where Darwin was stalled but what will conform with the laws made clear in this work.

Darwin is to be credited with being a true student of animal life, and a truthful historian when considered within the range of what he has encountered during his studies as to distribution, modes of life and general characteristics of the various orders. But as to the law at the base of the grand work, he was a child laboring in a world of mystery; the further he prosecuted his researches in to law the more he multiplied his want of knowledge.

The law of precipitation of matter within the temperature consistent with the mingling into the organic compound which goes toward forming into life, means life in all communities where the temperature becomes manifest in the cooling process. It means life as varied as the change of temperature capable of being 


\section{DARWINISM.}

made manifest by the topographical conditions. Where the topographical conditions are such as to bring into life or being, all the varieties, then all forms of life. Of course there is another question that would lessen certain orders of life in communities, that being lack of the mineral properties in the desired quantities. Men of one continent never were in any way connected with the men of another in the making. If there should be any crossing in the different families it must come after one family moving to the domains of the other. The same applies to all forms of life. The English were in no way related to eastern families of men until historic periods, they being native to the soil. The same will apply to all other nations where the topographr of the country will conform to the advent of men in the cooling process. Since the historic period they mixed by the entry of other families of men. But the same conditions of temperature were necessary to the advent of men in all localities; and hence they will cross between each other.

There is one point where Darwin deserves to be remembered throughout the ages, that point being his superceding a false god who had assumed control over mankind. The god which Darwin found enthroned in the fullest of powers over mankind was a false and tyrannical king of the dead ages, who dared to command all men to dare inquire into life, by might of their respective senses.

Darwin, knowing the weakness of men looking for miracles, gave them a few greater miracles than the 


\section{HEAT AND COLD.}

great miracle God could posisbly cope with. Darwin put a small worm on the throne, and the mighty worm made millions of great species out of nothing. The former god made man and a few other articles for man's use. Darwin's god displaced the old god, owing to having nothing to work with but himself, a measly worm, and he entangled himself throughout space and time until he worked out millions of great bodies of living, breathing, moving, growing and flowering organisms. All out of nothing. Darwin's god did all these miracles in the light of an advanced scientific world. Men read, they believed and were convinced. They knew the world was cooling and life was growing colder with the earth. They knew that it took more heat to keep a large organic property alive and in motion than it would to create the same action within a small body. They knew that nothing could be made out of nothing. But they immediately fell in line worshiping one miracle when it proved to be greater than the former miracle.

All men knew, in the light of their own senses, that Darwin's god was just as impossible as the god it displaced, but it was miraculous, and being more miraculous, owing to having a smaller body, and it being the father of the man god, of course in that case the god who sat enthroned could not hold the chair when the worm made him. The worm demanded the chair and the sulky little king who dared to assume the seat moved out. So all men became lovers of the great miraculous god who could make millions of organisms 


\section{DARWINISM.}

out of nothing. He not only made all organisms but made the tyrant who sat enthroned for centuries.

It would be well to call the reader's attention to the chapter on hybrids in Darwin's works, so he may observe how carefully law which is true law will prove itself. That he may observe that nature's law never varies or lies.

Darwin goes on to show by all his experiments, and that of others who had experimented up until the time of the writing of his work, that there can be a cross between various species. But the offspring is very generally sterile. When not sterile it breeds back to one of the progenitors. Thereby precluding any chance of bringing into being another species which can continue.

Now, it would be well to call the reader's attention to the fact that his experiments prove conclusively and beyond a reasonable doubt, the law of the constant in the forming process as outlined in this work. The constant arrived at between two species being capable of bringing into being a form of life. The form of life being incapable of continuation, owing to being an intermediate between the lives formed in the cooling process, and therefore having no law of continuation at its foundation.

Nature having had all the temperatures present in the cooling process, and in consequence brought into being all forms of life capable of pro-creating kind. Nature leaving no void as it had so many means of carrying on its work. Thereby nature left no room 


\section{HEAT AND COLD.}

for improvement in the number of procreating diversities. But the law of constant temperature applied to the plastic property at the foundation of life leaves room for the crossing of species.so as to bring life into being which must be ephermal in its continuation, owing to having no method of procreation other than an illegal union during each generation. Thereby such life is only conditional; and the conditions must be resorted to during the life time of each generation. Such life depends on two different beings each time it comes into existence. If it continues to procreate it does so by reversion to one form of progenitor, and loses all the properties derived from the other. Thereby precluding any chance to add to nature's work. 


\section{CONCLUSION.}

Having carried the same law at the foundation to inquiry within the various subjects, and having found that it is equal to the occasion in accounting for every phenomenon. It does not appear reasonable that the reader will doubt the truth of the law being all that the writer claims it to be, especially when you come to consider the various subjects which are, apparently, no way connected with each other, and each of the subjects surrounding truth when questioned within the code of inquiry.

Subjects that the reader would naturally believe to be impossible of solution within any one law; yet they all surrender to the one when questioned within the true order of its working method revealed to all in the light of their respective senses.

The law being one in which the inquirer will have to search for a condition in which it will not apply, instead of searching for condition on which to carry. out a false deduction. The author ventures to say that man cannot find a condition in nature where the law will not apply. More than that, man cannot find any method of action, movement or change capable of being applied by his own handiwork, but what will have to conform to the working law as herein described. He not being able to construct any mechanical contrivance of any form but what will have its foundation in the law herein described. 



\section{CONTENTS}

Page

Introductory ............................................................................................. 5

Chapter I-The Beginning of Things Earthly.................................. 9

Chapter II-Gravity....................................................................... 23

Chapter III-The Revolution of the Earth on its Axis, weighed

in the Light of Present Conditions of Surface...

Chapter IV-The Voyage of the Earth around the Sun and its

Effect upon Climate

Chapter V-Ocean Currents................................................................... 50

Chapter VI-Observations on the Movements of the Tide........ 56

Chapter VII-Earthquakes ........................................................................... 60

Chapter VIII-Cataclysms-Their Cause and Effect. Glacial

Epoch and Geological Confirmation Surveyed......................... 63

Geological Conditions................................................... 67

Chapter IX-Matter, Change of Form, Application of Measure 72

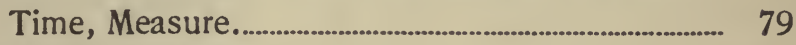

Chapter X-The Age of Water............................................................... 80

Chapter XI-Volcanoes .................................................................................... 84

Chapter XII-Organic Life in the Beginning, including Man and Other Life.

Chapter XIII-Organic Life and Planetary Conservation.......... 107

Chapter XIV-Man's Place in the Perpetuation of the Planet.. 108

Chapter XV-The Advent of Man and his Development............ 116

Chapter XVI-Circulation of the Blood................................................. 137

Chapter XVII-Second Period of Intellectual Development in

Man-Development of the Stomach-Feed for the Wolves

Clothing .

Chapter XVIII-The Office of the Brain.

Chapter XIX-Temperature vs. Intellect. 
Chapter XX-Morality

Legend of the Ark........................................................... 174

Chapter XXI-The God of Three Divine Persons........................ 180

Chapter XXII—Precedent........................................................................ 186

Chapter XXIII-Christ................................................................................. 192

Chapter XXIV-Bird and Animal Life.............................................. 201

Chapter XXV-Dangers to Human Organism................................. 213

Chapter XXVI-Death.......................................................................... 216

Chapter XXVII-Revolution of the Moon Around the Earth..... 222

Chapter XXVIII-Infinity of Foci, or the Generation of Suns.. 231 Duration of Suns................................................. 235

Chapter XXIX-Conformation of Planets.................................... 241

Duration of Planets................................................. 247

Chapter XXX-Darwinism ................................................................... 250

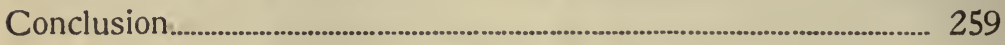





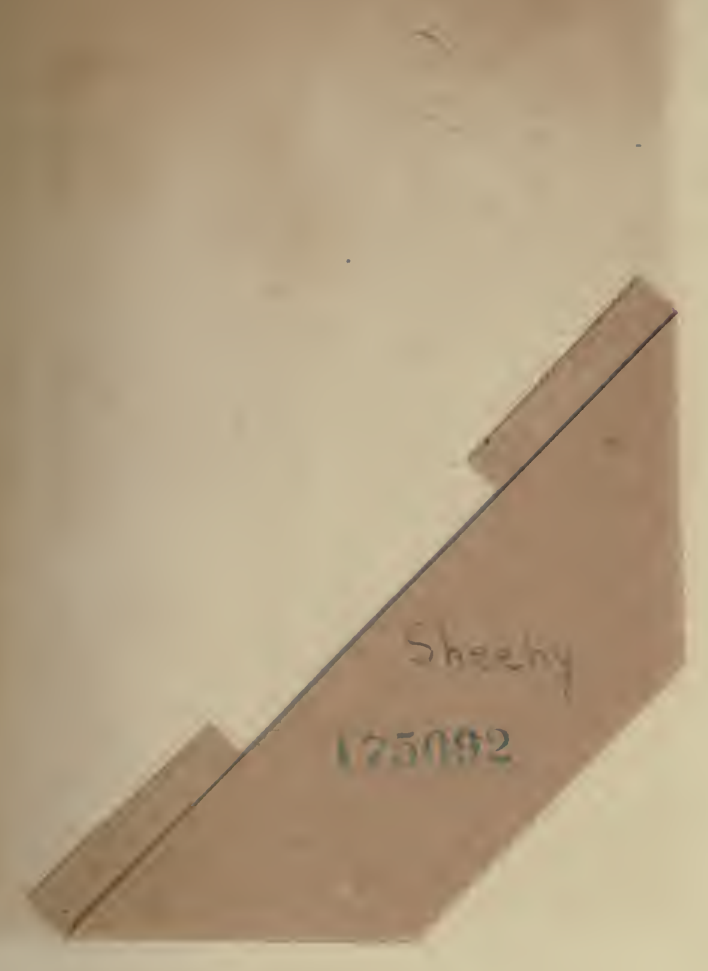


